\title{
Dusp6 Depletion Enhances Gut Barrier Integrity and Microbiota Eubiosis Against Dextran Sulfate Sodium-Induced Colitis in Mice
}

Cherng-Shyang Chang

National Health Research Institutes

Yi-Chu Liao

National Health Research Institutes

Chih-Ting Huang

National Health Research Institutes

Chiao-Mei Lin

National Health Research Institutes

Chantal Hoi Yin Cheung

National Taiwan University

Jhen-Wei Ruan

National Cheng Kung University

Wen-Hsuan Yu

National Taiwan University

Yi-Ting Tsai

National Tsing Hua University

Hsueh-Fen Juan

National Taiwan University

Hsuan-Cheng Huang

National Yang-Ming University

Hong-Lin Chan

National Tsing Hua University

Yu-Chieh Liao

National Health Research Institutes

Yu-Wen Su

National Health Research Institutes

Tse-Hua Tan

National Health Research Institutes

CHENG-YUAN KAO ( $\square$ chengyuankao@nhri.edu.tw)

National Health Research Institutes https://orcid.org/0000-0003-0946-9270 
Research

Keywords: Gut microbiota, Inflammatory bowel disease, Experimental colitis, Barrier integrity, Leaky gut

Posted Date: July 22nd, 2020

DOl: https://doi.org/10.21203/rs.3.rs-44154/v1

License: (c) (i) This work is licensed under a Creative Commons Attribution 4.0 International License.

Read Full License 


\section{Dusp6 Depletion Enhances Gut Barrier Integrity and Microbiota Eubiosis}

\section{Against Dextran Sulfate Sodium-Induced Colitis in Mice}

Cherng-Shyang Chang, ${ }^{1}$ Yi-Chu Liao, ${ }^{2,}$ \# Chih-Ting Huang, ${ }^{1,}$ \# Chiao-Mei Lin, ${ }^{1, ~ \# ~ C h a n t a l ~ H o i ~ Y i n ~}$ Cheung, ${ }^{3, \#}$ Jhen-Wei Ruan, ${ }^{4}$ Wen-Hsuan Yu, ${ }^{5}$ Yi-Ting Tsai, ${ }^{6}$ Hsueh-Fen Juan, ${ }^{3,5}$ Hsuan-Cheng Huang, ${ }^{7}$ Hong-Lin Chan, ${ }^{6}$ Yu-Chieh Liao, ${ }^{2}$ Yu-Wen Su, ${ }^{1}$ Tse-Hua Tan ${ }^{1}$ and Cheng-Yuan Kao ${ }^{1 *}$

${ }^{1}$ Immunology Research Center, National Health Research Institutes, Zhunan, Miaoli, 35053,

Taiwan

${ }^{2}$ Institute of Population Health Sciences, National Health Research Institutes, Zhunan, Miaoli, 35053, Taiwan

${ }^{3}$ Department of Life Science, National Taiwan University, Taipei, 10617, Taiwan

${ }^{4}$ Department of Medical Laboratory Science and Biotechnology, National Cheng Kung University, Tainan, 70101, Taiwan

${ }^{5}$ Graduate Institute of Biomedical Electronics and Bioinformatics, National Taiwan University, Taipei, 10617, Taiwan

${ }^{6}$ Institute of Bioinformatics and Structural Biology and Department of Medical Sciences, National Tsing Hua University, Hsinchu, 30013, Taiwan

${ }^{7}$ Institute of Biomedical Informatics, National Yang-Ming University, Taipei, 11221, Taiwan 
\# These authors contributed equally to this work.

* Correspondence and requests for materials should be addressed to C.Y.K. (email:

chengyuankao@nhri.edu.tw)

\section{Conflict of interest}

The authors have declared that no conflict of interest exists. 


\section{Abstract}

Background: Leaky gut and microbiota dysbiosis have been linked to many chronic inflammatory diseases. Strengthening the gut epithelial barrier is a novel but overlooked strategy for management of gut microbiota-associated illnesses.

Results: Using the dextran sulfate sodium (DSS)-induced gut barrier injury-based colitis model, we found that DSS-induced weight loss, rectal bleeding, and colonic epithelium damage were ameliorated in dual-specificity phosphatase 6 (Dusp6)-deficient mice. These protective effects could be attributed to the enhanced colon barrier integrity conferred by Dusp6-deficiency. Consistently, DUSP6 mutation in Caco-2 cells elevated transepithelial electrical resistance, enhanced tightjunctions, and increased expression of microvilli-associated genes. DUSP6-deficient Caco-2 cells also showed increased mitochondrial oxygen consumption accompanied by altered glucose metabolism and decreased glycolysis. Remarkably, our microbiome analysis found that Dusp6deficient mice harbored fewer pathobionts and facultative anaerobes and more obligate anaerobes than wild-type mice after DSS treatment. Our cohousing and fecal microbiota transplantation experiments demonstrated that the gut/fecal microbiota derived from Dusp6-deficient mice also conferred protection against colitis.

Conclusion: We have thus identified Dusp6 deficiency as beneficial in enhancing gut barrier integrity, elevating epithelial phosphoxidation, and maintaining the gut microbiota eubiosis necessary to protect against colitis. 
Keywords: Gut microbiota, Inflammatory bowel disease, Experimental colitis, Barrier integrity,

Leaky gut 


\section{Background}

Compromised gut barrier integrity has been associated with many chronic inflammatory diseases, as barrier formation is a crucial function of gut epithelial cells. Through apical junctional complex (AJC) substructures, including the adherens and tight junctions (TJs), gut epithelial cells link together and establish a boundary that limits entry of digested food and gut microbes into the circulation [1-3]. The transcellular pathway is therefore the main route for nutrient absorption, and gut epithelial cells act as the frontline in response to luminal microbes. The enterocytes express pattern recognition receptors (PRRs) to sense microbe-associated molecular patterns (MAMPs) and promote immune responses, including the production of anti-microbial peptides, the transportation of secretory $\lg \mathrm{A}(\mathrm{S} \lg \mathrm{A})$, and recruitment of immunocytes [4]. In addition, epithelial cells lining the gut are known to limit oxygen diffusion from the lamina propria into the intestinal tract, thereby establishing an anaerobic lumen for the growth of commensal anaerobes [5]. Enterocyte metabolism has also been found to mediate oxygen utilization, which can further limit the amount of oxygen that diffuses into the gut lumen [6].

Inflammatory bowel disease (IBD) refers to two chronic inflammatory digestive tract disorders: Crohn's disease and ulcerative colitis (UC). IBD is a globally emergent disease with accelerating incidence in newly-industrialized countries with increasingly Westernized lifestyles, while incidence is stabilizing at a high burden of disease in Western and other industrialized countries. The onset and progression of IBD is multifaceted and associated with a combination of genetic, microbial, and environmental factors. IBD risk has been linked to more than 200 genetic loci that affect host- 
microbiome interaction, most of which are related to cell-intrinsic barrier and key immunological pathways. For example, polymorphisms in C1orf106 increase susceptibility to IBD and C1orf106 has been shown to regulate epithelial cell surface levels of E-cadherin and adherens junction stability, which affect the intestinal epithelial cell barrier [7-9]. $C D H 1$, the gene that encodes E-cadherin, is also associated with an increased risk of UC [10]. Another UC-specific susceptibility gene is LAMB1, which encodes the laminin $\beta 1$ subunit $[10,11]$. Laminins interact with integrins to create a critical cell adhesion network in the intestinal epithelium, suggesting enhancement of gut epithelium integrity as a potential therapeutic strategy for IBD.

Intestinal barriers shape microbiota through multiple mechanisms [12] and, reciprocally, gut commensals aid in reinforcing gut barrier integrity [13]. In recent years, higher abundance of Proteobacteria and reduced diversity of the gut microbiome have become increasingly common in cases of IBD [14]. Although eubiosis and dysbiosis of gut microbiota have been shown to play important roles in the etiology of this disease, whether dysbiosis is an early event in IBD pathogenesis remains unclear due to the complex interactions of the host's genetics that affect barrier functions, the immune system, and gut microbiota [15]. Nevertheless, diet and probiotics may play important roles in regulating the gut microbiome and have been explored as supplementary strategies to manage the course of IBD. Another gut microbiome-centered strategy with a potential therapeutic impact in IBD is fecal microbiota transplantation (FMT). However, FMT studies conducted on IBD patients thus far have shown variable and inconclusive results. Donor-recipient compatibility, which requires consideration of genetic and environmental factors, may be critical in 
determining the likelihood of FMT success.

An emerging concept is the importance of gut barrier integrity in maintenance of overall health [1]. However, there have yet to be any FDA-approved therapeutic agents designed to improve gut epithelial barrier integrity $[16,17]$. Identification of a modulator with a vital role in regulation of gut epithelial function is imperative to improve management of intestinal diseases. We previously demonstrated that knockout of the Dusp6 gene, encoding Dual-Specificity Phosphatase 6 (DUSP6), a molecule involved in the negative feedback loop associated with Erk1/2 activation, ameliorated weight-gain after a high-fat diet, induced TJ pathway-related gene expression in the small intestine, and altered response of the gut microbiota to a high-fat diet in a mouse model [18]. We also found increased whole-body oxygen consumption in mice with Dusp6 deficiency. This evidence led to our hypothesis that Dusp6 depletion enhances gut epithelial barrier integrity and modulates the composition of the microbiota, providing a potential therapeutic target in the management of intestinal diseases. In this study, we applied multi-omics, cell biology, and gnotobiotic approaches using human gut epithelial Caco-2 cells and the DSS-induced experimental colitis model in Dusp6 gene-deficient mice to explore causative links between enhanced TJs, altered gut epithelial metabolism, and microbiome composition. We further examined whether these molecular alterations resulted in protection against DSS-induced damage to the gut epithelium in an animal model. Our microbiome analysis demonstrated that colitis-induced expansion of Proteobacteria was ameliorated in Dusp6 knockout (D6KO) mice and provided proof-of-concept that FMT could prevent DSS-induced colitis in mice. Our results suggest that DUSP6 inhibition could be applied as a novel 
therapeutic strategy to enhance gut epithelial barrier integrity in the prevention or treatment of gut barrier-related diseases. 


\section{Results}

\section{Dusp6 deficiency protects mice from DSS-induced colitis}

We first analyzed DUSP6 gene expression in human colonic mucosa samples from patients with UC using the public transcriptome dataset GSE9452 from the Gene Expression Omnibus [19]. DUSP6 expression was significantly up-regulated in UC-inflamed colonic mucosa compared with healthy controls; however, DUSP6 induction was significantly blunted in samples of UC-noninflamed compared with UC-inflamed colonic mucosa (Figure 1A). Moreover, our reanalysis of a published single-cell RNA-seq dataset (Single Cell Portal: SCP259) of cells from the colonic mucosa of UC patients and healthy individuals [20] revealed that UC-induced DUSP6 gene expression was observed primarily in colonic epithelial cells (Figure 1B), rather than in immune or stromal cells

(Figure 1, C and D). We then investigated the effects of Dusp6 deficiency on intestinal epithelial injury using a murine model of dextran sulfate sodium (DSS)-induced colitis. After 9 days of treatment, immunohistochemical staining demonstrated colonic epithelial injury concurrent with increased DUSP6 expression in wild-type (WT) mice exposed to 2\% (w/v) DSS compared to controls (Figure 1E). Changes in Dusp6 expression in response to DSS-induced gut epithelial injury in mice appeared similar to effects observed in samples from patients with UC. Additional analysis of DSSinduced colitis in male D6KO mice showed attenuation of DSS-induced weight loss compared with DSS-treated WT littermates (Figure 1, F and G; Supplemental Figure 1A). Rectal bleeding and diarrhea caused by DSS in WT mice were ameliorated in D6KO mice (Supplemental Figure 1, B and C), leading to a lower disease activity index in DSS-treated D6KO mice compared to DSS- 
exposed WT controls (Figure 1H). Moreover, DSS-treated D6KO mice showed greater water consumption and food intake than DSS-treated WT mice (Supplemental Figure 1, D and E), suggesting that the D6KO mice had reduced disease severity and discomfort in response to DSS. Although D6KO mice showed no significant protection against DSS-induced splenomegaly or colonic cytokine production (Supplemental Figure 2, A and B), DSS-induced shortening of colon length in D6KO mice was attenuated (Figure 1, I and J). Thickening of the outer muscle layer of the colon was observed in both D6KO and WT mice after DSS stimulation (Figure 1K, top row), and transverse sections showed significantly altered colonic luminal architecture and barrier in WT mice after DSS treatment. In contrast, the luminal epithelial layer appeared normal in DSS-treated D6KO mice. Distortion of crypts of Lieberkuhn (intestinal glands) were observed in WT mice after DSS treatment (Figure 1K, bottom row), accompanied by a distinct reduction in the number of goblet cells and infiltration of mononuclear cells (Figure 1L). None of these pathohistological defects were observed in DSS-treated D6KO mice, suggesting that Dusp6 deficiency conferred protection against DSS-induced colitis via maintenance of intestinal epithelium integrity.

We also explored whether sex was a factor contributing to the protective effects of Dusp6 deficiency against DSS-induced colitis. The body weight of female WT mice decreased after 9 days of treatment with DSS, whereas the weight of female D6KO mice was not significantly different from controls (Supplemental Figure 3, A and B). DSS-induced shortening of the colon was observed in female WT mice, but not in female D6KO mice (Supplemental Figure 3, C and D). Pathohistological changes in the colonic epithelium, including crypt distortion, goblet cell loss, and 
mononuclear cell infiltration were observed in female WT mice after DSS treatment, but not in DSStreated female D6KO mice (Supplemental Figure 3E). Therefore, female D6KO mice exhibited the same protection from DSS-induced colitis as male D6KO mice.

\section{Dusp6 deficiency enhances intrinsic colonic tight junctions and elongates microvilli in mice}

Dysregulation of the gut epithelial barrier has been linked to colitis; therefore, we examined TJs within the colonic epithelium in mice. Using quantitative real-time PCR (qRT-PCR) of mRNA expression of a panel of colonic TJ proteins, we found that the baseline mRNA levels of Tjp1, Tjp2, and OcIn were significantly higher in the colonic tissue of D6KO mice compared with that of WT mice (Figure 2A). The basal level of TJP1 was higher in the colonic epithelium of D6KO mice compared with WT littermates, as demonstrated by immunofluorescent staining of TJP1 protein on the epithelial surface along the lumen and crypt axes (Figure 2B, Water). Similarly, TJP2 was more abundant in colonic epithelial cells of D6KO mice compared to WT, and immunofluorescent images showed TJP2 protein localization on both the epithelial membrane and throughout the cytoplasm. Although the level of the Tjp3 transcript was not altered by Dusp6 deficiency, immunofluorescent staining displayed more extensive expression of TJP3 in the colonic epithelium of D6KO mice compared with WT (Figure 2, A and B). These results suggest that Dusp6 deficiency increased the overall production of TJ proteins in mice in the basal state. However, the levels of Tjp1 and Tjp2 mRNA in DSS-treated D6KO mice were decreased to a level similar to those of DSS-treated WT 
mice (Figure 2A), revealing that DSS treatment impeded the effects of Dusp6 deficiency on TJassociated gene induction. TJP1, TJP2, and TJP3 immunofluorescence was nearly undetectable at sites of colonic inflammation in DSS-treated WT mice due to the loss of epithelium (data not shown), whereas the intensity of protein-specific fluorescence at non-inflammatory sites was increased in WT mice after DSS treatment compared to WT controls (Figure 2B). These data suggest that the epithelial TJ proteins can be induced in a compensatory manner in regions not yet destroyed by DSS treatment. In addition, expression of TJP1, TJP2, and TJP3 was largely unaffected in the colonic epithelium of DSS-treated D6KO mice, and the fluorescence intensity of these proteins remained higher in D6KO mice than in WT mice after DSS treatment. Transmission electron microscopy also revealed that Dusp6 deficiency enhanced the electron density of colonic TJ complexes (Figure 2C), and increased colonic epithelial cell microvilli length was observed in D6KO mice compared to WT (Figure 2, C and D). We further assessed the mRNA levels of a panel of microvilli-associated genes and found that basal expression of Vil1, Vill, Myo5b, and Myo5c was significantly increased in the colonic tissue of D6KO mice compared with that in WT controls (Figure 2E, Water). Overall, Dusp6 deficiency was found to increase intestinal barrier integrity through TJ enhancement and microvilli elongation.

\section{DUSP6 deficiency alters the transcriptome and phospho-proteome in Caco-2 cells}

The activation of gut barrier genes observed in D6KO mice may be due to intrinsic effects of gene deletion or exogenous factors such gut microbiota. To investigate whether DUSP6 deficiency 
directly enhances gut epithelial barrier integrity, we examined the effects of DUSP6 knockout on barrier function and the expression of barrier-associated genes in human gut epithelial Caco-2 cells.

A nonsense DUSP6 gene mutation that blocked DUSP6 protein expression was introduced into Caco-2 cells (DUSP6 KO) in our previous study [18]. Transepithelial electrical resistance (TEER) was significantly increased in DUSP6 KO Caco-2 cells compared with WT Caco-2 cells (Supplemental Figure 4A). Using RNA-seq transcriptome profiling, we found 1730 significantly upregulated genes and 1501 significantly down-regulated genes in DUSP6 KO Caco-2 cells compared with WT Caco-2 cells (Figure 3A). We then used FunRich software to analyze the differentially expressed genes (DEGs) with Gene Ontology (GO) enrichment analysis and found that DUSP6 KO Caco-2 cells had enrichment of up-regulated genes associated with 9 biological processes and enrichment of down-regulated genes associated with 4 biological processes (Supplemental Figure 4B). Both up-regulated and down-regulated genes enriched in DUSP6 KO Caco-2 cells were predominantly involved in energy and metabolism pathways, which suggested that DUSP6deficiency significantly altered cellular metabolism and energy flow. We then identified potential biological pathways regulated by DUSP6 in gut epithelial cells using FunRich biological pathway analysis, which resulted in 68 pathways enriched in up-regulated DEGs and 116 pathways enriched in down-regulated DEGs from DUSP6 KO Caco-2 cells (Figure 3B). Among the 68 up-regulated pathways, we found several biological pathways potentially relevant to the effects of colitis, including mesenchymal-to-epithelial transition (MET), interferon alpha/beta signaling, and hypoxic and oxygen homeostasis regulation of hypoxia inducible factor (HIF)-1-alpha. The epithelial-to- 
mesenchymal transition (EMT) pathway showed enrichment in down-regulated DEGs in DUSP6 KO Caco-2 cells. The biological context of concurrent MET-stimulation and EMT-repression in DUSP6 KO Caco-2 cells implied that improved intrinsic epithelium barrier function and enhancement of cell polarity are mediated by DUSP6 deficiency.

DUSP6 is a phosphatase, and therefore it is plausible that DUSP6 knockout alters the phosphorylation status of other proteins. To assess the protein phosphorylation landscape in DUSP6 KO Caco-2 cells, we performed phospho-proteomic profiling using a dimethyl labeling method [21]. A total of 1323 phosphosites on 580 phosphoproteins were identified, among which 61 downregulated and 84 up-regulated phosphoproteins had $\geq 1.5$-fold change in expression in DUSP6 KO Caco-2 cells compared to the WT Caco-2 cells (Supplemental Table 2). An additional biological process and pathway analysis using FunRich showed that DUSP6 knockout largely altered the enrichment of the cellular signaling-related phospho-proteome (Figure 3C; Supplemental Figure 4C; Supplemental Table 3). Among these, 34 biological pathways overlapped with pathways comprising the down-regulated DEGs enriched in DUSP6 KO Caco-2 cells (Supplemental Figure 4D), most of which were associated with cellular signaling. We also found that 10 of the pathways enriched in the altered phospho-proteome overlapped with pathways comprising the up-regulated DEGs enriched in DUSP6 KO Caco-2 cells (Figure 3D). Concurrent identification of MET and hypoxic and oxygen homeostasis regulation of HIF-1-alpha pathways implied that epithelial barrier integrity, as well as cell polarity enhancement and oxygen homeostasis, might be modulated by DUSP6 deficiency via both gene expression and protein phosphorylation. Functional annotation by 
GO enrichment analysis also revealed that DUSP6 deficiency altered the phosphorylation status of proteins associated with cell signaling, proliferation, differentiation, apoptosis, the cytoskeleton, adhesion, cell-cell junctions, and carbohydrate metabolism (Supplemental Figure 5A). Subsequent Kyoto Encyclopedia of Genes and Genomes (KEGG) analysis with D.A.V.I.D. further indicated the TJ pathway as significantly enriched in DUSP6 KO Caco-2 cells via phospho-proteomic profiling (Supplemental Figure 5B).

Further examination by qRT-PCR revealed that the expression of TJP1, TJP2, TJP3, OLCN, CLDN3, and CLDN7 was induced by DUSP6 deficiency (Figure 3E). Western blotting showed that TJP1 and TJP2 expression was induced in DUSP6 KO Caco-2 cells (Figure 3F), although no increase in TJP3 expression was observed in comparison with WT cells. Immunofluorescent staining showed that TJP1 was expressed at the plasma membrane in both WT and DUSP6 KO cells, with a higher intensity and a sharper staining pattern in DUSP6 KO cells (Figure 3G). The intensity of TJP2 staining was dramatically higher in DUSP6 KO cells compared with WT cells, whereas the staining intensity of TJP3 was only slightly higher in DUSP6 KO cells compared to WT. We also assessed the mRNA levels of the panel of microvilli-associated genes and found that expression of VILL, PLS1, MYO5A, MYO5C, MYO7A, and MYO7B was significantly increased in DUSP6 KO cells compared to WT cells (Figure $3 \mathrm{H}$ ). The evidence indicated that DUSP6 gene depletion alone could cause induction of TJ and microvilli-associated genes, leading to enhanced barrier integrity in vitro without the influence of gut microbiota. Together, these results indicate that DUSP6 deficiency might 
enhance the TJ pathway at different levels, including those of gene expression, protein expression, and phosphorylation.

\section{DUSP6 deficiency induces mitochondrial phosphoxidation and reduces glycolysis}

Both transcriptome and phospho-proteome profiling suggested that hypoxia and oxygen homeostasis regulation via HIF-1-alpha pathways were affected by DUSP6 deficiency (Figure 3D). Therefore, we investigated the link between oxygen utilization and metabolism in DUSP6 KO Caco2 cells. Induction of hypoxia-associated gene expression was observed at both the mRNA and protein levels (Figure 4, A and B), which suggested a state of hypoxia and increased oxygen utilization in DUSP6 KO Caco-2 cells. Using a Seahorse Analyzer, we found that DUSP6 KO cells had a higher basal oxygen consumption rate than WT cells (Figure 4C). Increased mitochondrial ATP production and decreased spare respiratory capacity were also found in the DUSP6 KO cells compared to WT cells (Figure 4D), suggesting that DUSP6 KO cells relied on mitochondrial oxidative phosphorylation to maintain energy homeostasis. However, we found less change in $\mathrm{pH}$, as indicated by culture medium color change from red (basic) to yellow (acidic) after incubation of DUSP6 KO Caco-2 cells compared to WT cells (Figure 4E). A change from glycolytic to more aerobic, and simultaneously from a more metabolic to a less metabolic state, was observed in the cells with DUSP6 gene knockout (Figure 4F). Although the extracellular acidification rate (ECAR) was comparable between WT and DUSP6 KO cells in the glucose starvation state, the DUSP6 KO cells showed a lower ECAR than WT cells after addition of glucose (Figure 4G), indicating a 
decreased glycolysis rate in DUSP6 KO cells (Figure 4H). Inhibition of mitochondrial ATP synthase by oligomycin increased the demand for cytosolic glycolysis and led to ECAR elevation. Similarly increased ECARs were found in WT and DUSP6 KO cells after oligomycin supplementation, and the reserve capacity for glycolysis was not altered by DUSP6 knockout. These results suggested that the glycolytic machinery remained functional in DUSP6 KO cells in response to higher demand for glycolysis. However, a lower maximal glycolytic capacity was observed in DUSP6 KO cells compared with that in WT cells. Indeed, the phospho-proteomic profiling suggested that DUSP6 deficiency altered the phosphorylation status of some glucose metabolism proteins (Supplemental Figure 5). Our qRT-PCR analysis also showed that genes associated with carbohydrate processing, such as $\mathrm{SI}$, a crucial glucosidase for digestion of starch in the gut lumen [22], as well as SGLT1 and GLUT2, the major glucose transporters in gut epithelial cells $[23,24]$, were down-regulated in the context of DUSP6 deficiency (Figure 4I). An induction of cellular ATP production was found in WT Caco-2 cells after addition of glucose for 4 hours (Figure $4 \mathrm{~J}$ ). In contrast, no elevation of cellular ATP was found in DUSP6 KO Caco-2 cells after glucose stimulation. Collectively, these results indicate that DUSP6 deficiency in Caco-2 cells leads to reduced glycolysis and induction of mitochondrial phosphoxidation, which may further enhance hypoxia in the micro-environment that shapes the gut microbiota.

\section{The gut microbiota of Dusp6 knockout mice in DSS-induced colitis}

Besides gut barrier integrity, gut microbiota have been shown to play important roles in IBD [14, 
25-27]. Reanalysis of the microbiome dataset from the NIH Human Microbiome Project demonstrated that IBD subjects harbored more Proteobacteria, Firmicutes, Fusobacteria and Actinobacteria, and less Bacteroidetes than non-IBD subjects (Figure 5A). Therefore, we analyzed the fecal/gut microbiota of D6KO mice and WT littermates with DSS-induced colitis. After 9 days of treatment with drinking water containing DSS, the fecal/gut microbiota richness, Shannon diversity, and evenness were decreased in WT mice compared to untreated (water only) control mice (Supplemental Figure 6A). While microbiota richness was also reduced in D6KO mice after DSS treatment compared to untreated controls, the Shannon diversity and evenness indices remained comparable to controls (Supplemental Figure 6A). These data suggest that Dusp6 deficiency protects and promotes gut microbiome eubiosis even under considerable chemical-induced colonic stress. Further Principal Coordinates Analysis (PCoA) based on the distance matrix of Bray-Curtis dissimilarity showed a significant difference in the fecal/gut microbiome between D6KO mice and WT littermates both before and after DSS treatment (PERMANOVA/Adonis test, $P=0.01199$ and $P=0.003996$, respectively; Supplemental Figure 6, B and C). The abundance of phylum Proteobacteria, commonly classified as inflammophilic pathobionts, increased substantially in the gut microbiome of WT mice after DSS treatment (Figure 5B; Supplemental Table 4). However, the abundance of Proteobacteria in the gut microbiome of D6KO mice was only slightly increased by DSS treatment. At the family level, the abundance of Enterobacteriaceae was largely elevated in DSS-treated WT mice $(12.73 \pm 2.09 \%)$ compared to the untreated WT controls $(0.00 \pm 0.00 \%)$. In contrast, Enterobacteriaceae abundance was only slightly increased in DSS-treated D6KO mice 
$(4.01 \pm 1.26 \%)$ compared to untreated D6KO controls $(0.00 \pm 0.00 \%$; Figure $5 \mathrm{C}$; Supplemental

Table 4). We further confirmed that the expansion of the Enterobacteriaceae family was primarily due to a greater abundance of Escherichia, a genus of facultative anaerobes (Supplemental Table 4). Similarly, expansion of other facultative anaerobes, such as Enterococcus and Streptococcus, was observed in DSS-treated WT mice, whereas comparatively smaller increases in the abundance of these bacteria were found in DSS-treated D6KO mice (Supplemental Table 4). Numbers of obligatory anaerobes, such as Muribaculaceae, were relatively preserved in DSS-treated D6KO mice, whereas significant loss of Muribaculaceae was observed in DSS-treated WT mice (Figure 5C; Supplemental Table 4). Phenotypic analysis using BugBase revealed that the abundance of oxygen-utilizing bacteria, including aerobes and facultative anaerobes, increased substantially in DSS-treated WT mice compared to WT controls (Figure 5D; Supplemental Table 5). In contrast, the gut microbiota comprising a greater proportion of obligatory anaerobes was preserved in DSStreated D6KO mice compared to D6KO controls. We also applied linear discriminant analysis effect size (LEfSe) to the microbiome data and confirmed that the abundance of Escherichia, Enterococcus, and Streptococcus was significantly higher in WT mice compared with D6KO mice after DSS treatment (Figure 5, E and F). Very low abundance of these bacteria was found in WT and D6KO mice before DSS treatment (Supplemental Table 4; Supplemental Figure 7), suggesting that those bacteria opportunistically expanded in DSS-treated WT mice, whereas expansion was restricted in DSS-treated D6KO mice. Further microbiome analysis at the species level, via aligning the microbial amplicon sequence variants (ASVs) against the NCBI database, 
revealed that the biological context of DSS-treated WT mice favored certain pathobiont expansion, whereas restriction of the same pathobionts was observed in DSS-treated D6KO mice (Figure 5G). The Streptococcus spp., the pathobiont that naturally resides in the upper digestive or respiratory tracts, was found in DSS-treated WT mice. The abundance of Escherichia fergusonii, a pathobiont associated with intestinal and extra-intestinal infections in both humans and animals [28], was significantly increased in WT mice after DSS treatment but not in DSS-treated D6KO mice. The abundance of Enterococcus faecalis and Enterococcus hirae, a cause of diverse infectious diseases $[27,29,30]$, was also increased in WT mice after DSS treatment, but restricted in DSS-treated D6KO mice. The abundance of Rodentibacter pneumotropicus, a pathobiont in laboratory animals [31], was elevated in DSS-treated WT mice, but D6KO mice showed reduced expansion after treatment with DSS. The abundance of Ruminococcus gnavus, an IBD associated bacteria [14, 25, 26, 32], was significantly increased in DSS-treated WT mice, and expansion was limited in DSS-treated D6KO mice. Contrary to the general pattern were the colitis-associated bacteria Bacteroides acidifaciens, the abundance of which was increased in both WT and D6KO mice after DSS treatment, and Bacteroides caecimuris, which has been reported to be elevated in the recovery phase after discontinuing DSS treatment [33], and was increased by nearly 2-fold in D6KO compared to WT mice after DSS treatment. This evidence demonstrated aberrant composition of gut/fecal microbiota of WT mice after DSS treatment, whereas expansion of the pathobionts was generally restricted in DSS-treated D6KO mice. 
The gut microbiota from Dusp6 knockout mice contribute to protection from DSS-induced colitis

In our previous study, we found that Dusp6 deficiency could protect mice from gut dysbiosis induced by nutritional stress [18]. To investigate whether microbiota-dependent machinery contributed to counteraction of chemical-induced stress on the gut barrier in D6KO mice, we setup cohousing experiments in which WT and D6KO mice were caged together for 4 weeks immediately after weaning, then treated with water alone or water with DSS to induce colitis (Figure 6A). We found that DSS-induced loss of body weight improved among WT mice cohoused with D6KO mice (Figure 6B). The colitis disease activity index was reduced in WT mice cohoused with D6KO mice (Figure 6C) compared to non-cohoused mice, an effect that was accompanied by recovery of colon length (Figure 6D). The luminal architecture, mucus production, and crypt structure of the colonic epithelium were relatively normal in the cohoused versus non-cohoused WT mice (Figure 6, E and F). In addition, the protective effects of Dusp6 deficiency on DSS-induced colitis were sustained in D6KO mice after cohousing with WT mice. The analysis of fecal/gut microbiota from DSS-treated mice revealed that cohoused WT mice harbored a lower abundance of phylum Proteobacteria compared with non-cohoused WT mice (Figure 6G; Supplemental Table 6). At the family level, lower abundance of family Enterobacteriaceae, Enterococcaceae, and Streptococcaceae bacteria, and greater abundance of Muribaculaceae were found in cohoused WT mice compared with noncohoused WT mice (Figure 6H; Supplemental Table 6). LEfSe showed a significant increase of genus Muribaculum bacteria and significant decreases of family Enterococcaceae and genus 
Enterococcus bacteria in cohoused WT mice compared with non-cohoused mice (Supplemental

Figure 8). Accordingly, BugBase analysis revealed that the abundance of facultative anaerobes decreased in cohoused WT mice (Figure 6I; Supplemental Table 7). These results suggest that Dusp6 deficiency and gut microbiota-mediated mechanisms may interact to protect against colitis.

In addition to cohousing experiments, FMT in germ-free WT mice was performed to further demonstrate the beneficial effects of gut microbiota from D6KO mice. We transplanted the fecal microbiota from D6KO mice or WT littermates into germ-free B6 mice and conducted the DSSinduced colitis experiment 2 weeks after transplantation (Figure 7A). DSS-induced weight loss was observed in B6 mice that received fecal microbiota from WT mice, whereas less weight loss was observed in B6 mice that received fecal microbiota from D6KO mice (Figure 7B). The disease activity index showed reduced severity of DSS-induced colitis in the germ-free mice with gut microbiota from D6KO mice compared to those with gut microbiota from WT mice (Figure 7C). Similarly, DSS-induced colon shortening was attenuated in germ-free mice that had received gut microbiota from D6KO mice (Figure 7D). The colonic epithelium was damaged by DSS in the transplant recipients with fecal microbiota from WT mice (Figure 7, E and F). In contrast, DSSinduced damage to the colonic epithelium was mitigated in transplant recipients with gut microbiota from D6KO mice. Although the D6KO-derived microbiota did not induce the expression of TJassociated genes in germ-free mice that received normal drinking water, the levels of Tjp1, Tjp3, Ocln, Cldn3, and Cldn7 mRNA were significantly higher in DSS-treated mice that received D6KOderived microbiota than in those that received WT-derived microbiota (Figure 7G). Additionally, 
significant decreases in DSS-induced expression of some cytokines were observed in germ-free mice that received D6KO-derived microbiota (Figure 7H). Overall, these results demonstrate that D6KO-derived fecal microbiota has the potential to regulate gut homeostasis and protect against DSS-induced damage to the mouse colonic epithelium. 


\section{Discussion}

Through this study, we are the first to show that Dusp6 deficiency enhanced intrinsic gut barrier integrity and aided in maintaining anaerobic gut microbiota eubiosis, and that gut/fecal microbiota derived from Dusp6 deficiency conferred host resistance to DSS-induced colonic injury (Figure 8). We found that Dusp6 deficiency was protective against DSS-induced colonic injury, although Bertin et al. showed that Dusp6 restrained spontaneous colitis in IL-10-deficient mice [34]. Critical differences in experimental design may have contributed to these divergent results. Due to the complexity of human IBD pathogenesis, diverse experimental mouse models have been established to mimic specific potential causes of IBD, allowing investigation of mucosal immune function and therapies [35]. Our studies focus on the roles of Dusp6 in gut epithelium barrier integrity. We therefore chose the DSS-induced colitis model, as DSS causes direct colonic epithelium barrier injury. In contrast, Bertin et al. used the IL-10-deficient mouse colitis model, which is a canonic immunoregulation model focused primarily on macrophages and T cells. Divergent results are not unexpected given the roles of Dusp6 were assessed in different cell types using different mouse models. Additionally, gut microbiota are known to play critical roles in the development of colitis in some experimental strains; for example, germ-free IL-10-deficient mice do not develop colitis [36]. In the study by Bertin et al., the $1110^{-/-}$and $1110^{-/ /} / \mathrm{Dusp6}^{-/-}$mice were purposely cohoused starting at 4 weeks of age, and these cohoused mice were used for all experiments to control for the effects of gut microbiota [34]. The effects of gut microbiota may be another key factor contributing to the discrepant results compared to our study, as we found that the gut microbiota derived from Dusp6- 
deficient mice could confer host resistance to DSS-induced colonic injury. A recent report from Beaudry et al. also indicated that Dusp6-knockout mice are more resistant to DSS-induced colonic injury compared to WT mice [37], which is consistent with our findings. However, the Beaudry et al. report did not provide details regarding specific changes in the gut microbiota of Dusp6-deficient mice and how those alterations affect host susceptibility to DSS-induced colonic injury.

The colon is the terminal portion of the gastrointestinal tract, and a healthy colon is an environment of low oxygen concentration that harbors a population of approximately $10^{10-10^{13}}$ bacteria consisting of predominantly obligate anaerobes [32, 38]. In our study, we observed a marked expansion of Enterobacteriaceae, Enterococcus, and Streptococcus in the fecal microbiota of WT mice with DSS-induced colonic epithelial damage, whereas the abundance of these pathobiont facultative anaerobes was restricted in the fecal microbiota of DSS-treated D6KO mice. These data suggested involvement of Dusp6 in the regulation of intestinal oxygen and microbiota eubiosis. The reduction of obligate anaerobes and sharp expansion of facultative anaerobes has been a proposed signature of IBD [14, 39]. Furthermore, low oxygenation has been shown to affect gut microbial composition and metabolism [6, 40]. Intriguingly, a newborn gastrointestinal tract is known to contain a relatively high level of oxygen, which in turn favors colonization by facultative anaerobes, such as Enterobacteriaceae, Enterococcus, and Streptococcus [41]. While some of these facultative anaerobes have pathogenic potential [32,42], these early colonizers consume the luminal oxygen and create a healthy anaerobic micro-environment for subsequent expansion of obligatory anaerobes in the gut. Indeed, oxygen level has been linked to the virulence of intestinal 
pathogens [43], and low oxygen has been shown to increase the bactericidal activity of host cells $[44,45]$. Thus, Dusp6 deficiency may be beneficial in maintenance of low oxygenation in the gut lumen and lead to preservation and expansion of obligatory anaerobes, which may then decrease the risk of, and damage from, colitis.

Whether alteration of microbiota is a cause or a consequence of colitis remains debatable [32].

Our data showed that Dusp6 deficiency limited the growth of pathobiont facultative anaerobes and ameliorated DSS-induced damage to the colonic epithelium, yet it is still unclear whether the expansion of gut microbes identified in DSS-treated WT mice are contributing to the development of colitis. The increased abundance of facultative anaerobes may also be a consequence of epithelium leakage, which accelerates the release of oxygen from the lamina propria into the intestinal tract [32]. In other words, the lower abundance of facultative anaerobes in D6KO compared to WT mice after DSS treatment may have resulted from reduced severity of DSS-induced epithelial leakage. We showed that Dusp6-deficient intestinal epithelial cells have enhanced barrier integrity, which limits the materials, including gut microbes, oxygen, and DSS, that flow across the epithelium through an intercellular pathway. Further studies are necessary to elucidate whether DSS treatmentstimulated expansion of facultative anaerobes play a causative role in colitis and whether these events are directly restricted by Dusp6 deficiency.

HIF plays critical roles in maintaining intestinal barrier function, mucin production, and the expression of anti-microbial peptides in response to both infective and non-infective colitis [46]. HIF1a elimination in mice resulted in increased severity of Clostridium difficile-induced intestinal injury 
[47]. The lack of HIF-1 $\alpha$ in epithelial cells has been shown to enhance colitis in mice, whereas the constitutive activation of HIF-1a in colon epithelial cells demonstrated protective effects [48]. Interestingly, hypoxia and HIF-1 1 p protein stability have been associated with DUSP6 mRNA stability in both skin and colon cancer cells [49]. Hypoxia-induced DUSP6 mRNA stability has been shown to be regulated via HIF-1 and MEK/ERK dependent pathways [49]. However, we found that HIF-1 expression was up-regulated in DUSP6-deficient cells. DUSP6 may act as a brake not only for the Erk1/2 pathway but also for hypoxia and HIF-1 downstream signaling. Besides the up-regulation of HIF-1, we also observed up-regulation of TJ proteins and increased transepithelial electrical resistance in DUSP6-deficient Caco-2 cells under normoxia. This suggests that the hypoxic signals and HIF-1 may be continuously activated in DUSP6-deficient intestinal epithelial cells, thereby increasing intestinal barrier function.

Dysregulation of the gut epithelial barrier has been linked to many intestinal diseases $[1,3,17$, $50,51]$. The loss of TJ proteins [52] has been reported to increase susceptibility to DSS-induced colitis in mice. Moreover, enhanced barrier function has been shown to enhance resistance against colitis [53]. In this study, we demonstrated that Dusp6 deficiency induces expression of TJ proteins in the colon. This is consistent with our previous finding that Dusp6 deficiency led to increased expression of TJ proteins in the small intestine [18]. Therefore, the elimination of Dusp6 could be a strategy to enhance epithelial barrier function in both the colon and small intestine. The increased expression of TJ proteins associated with Dusp6 deficiency was generally observed in the treatmentnaive state. Immunofluorescent staining for TJP proteins was undetectable in DSS-damaged 
regions of the colon in WT mice due to epithelial loss. In contrast, we found that the intensity of TJP staining fluorescence in intact epithelial regions of the colon in WT mice was increased in a compensatory manner. These results suggest that the undamaged and yet to be damaged colonic epithelial cells increased barrier function in response to injury, despite timing that was too late to fully rescue epithelial function. In contrast, Dusp6 deficiency enhanced TJ-associated gene expression in treatment-naive epithelial cells, suggesting enhanced pre-treatment barrier integrity and protection from subsequent injury. This mechanism may explain the results of a recent pilot study in which the DUSP6 SNP rs704074 was associated with a significantly lower risk of developing necrotizing enterocolitis (NEC) and surgical NEC in preterm infants [54], further supporting the potential to use DUSP6 deficiency to protect against colitis.

Besides Dusp6 regulation of TJ pathway gene expression and protein abundance, the TJ pathway was found to be one of the most enriched KEGG pathways via phospho-proteomic profiling of DUSP6-deficient Caco-2 cells. Prior phospho-proteomic profiling of liver tissue from mice fed a high fat diet has also shown significantly increased phosphorylation of the TJ proteins TJP1 and TJP2 in Dusp6-knockout compared to WT mice [55]. Therefore, Dusp6 may be involved in the core regulation of the TJ pathway in other tissues (e.g., liver) in addition to the colon and small intestine. TJs function as a physiological frontline and maintain cell polarity in barriers consisting primarily of epithelial and endothelial cells. Emerging experimental evidence has suggested that the reduction of TJs may be associated with various diseases, in addition to intestinal ailments, including multiple sclerosis, diabetic nephropathy, asthma, atopic dermatitis, infection, and tumor metastasis [56]. It is 
possible that previously reported beneficial effects of Dusp6 deficiency, such as resistance to dietinduced obesity [18] and alleviation of endothelial inflammation [57] are also related to enhanced TJ pathway signaling and improved barrier integrity. Given the important roles of TJs in physiology and disease, our findings that Dusp6-deficiency enhanced TJ pathway signaling have shed light on the previously neglected therapeutic potential of enhancing barrier integrity by inhibiting DUSP6 expression. Remarkably, a pharmacological DUSP6 inhibitor, $\mathrm{BCl}$, has been reported to induce Ecadherin expression and reduce the migration and invasion of the gastric cancer cell lines SGC7901 and BGC823 [58].

Epithelial microvilli play critical roles in establishing the gut barrier. A recent transcriptome analysis reported previously unrecognized features of Crohn's disease, including the correspondence between decreased expression of microvilli-associated genes (e.g., VIL1, MYO5B, MYO7B, PLS1) and shortened length of intestinal microvilli [59]. Plastin 1 (encoded by PLS1) and Villin (encoded by VIL1) are the core components of microvilli. The PIs1-deficient mouse has shorter microvilli and increased sensitivity to DSS-induced intestinal injury [60]. Results of these studies and our data, which showed induction of microvilli-associated genes in DUSP6-deficient Caco-2 cells and/or D6KO mice, along with elongated colonic microvilli in D6KO mice, provide further evidence that enhanced baseline barrier integrity is conferred by Dusp6-deficiency.

FMT has been explored as a novel approach to correct the gut dysbiosis associated with many diseases, and several clinical trials of FMT as IBD treatment have been conducted. For UC, the results from two randomized trials showed that FMT was more effective than placebo control [61, 
62], but another randomized trial did not show any significant difference between FMT versus control

[63]. For Crohn's disease, FMT resulted in clinical improvement in a pilot study that enrolled pediatric patients and a small separate cohort of adults [64], whereas another randomized, single-blind pilot study showed that FMT did not result in clinical improvement but could serve as a maintenance treatment after corticosteroids [65]. Overall, the clinical evidence for the efficacy of FMT in the treatment of IBD is inconclusive, and another randomized clinical trial is ongoing (https://clinicaltrials.gov/ct2/show/NCT03078803). We found that commensal microbes derived from D6KO mice were protective to the host, as demonstrated in the cohousing experiments and through FMT in germ-free mice; and our results also indicated that acquired allogenic fecal microbiota, in addition to congenital genetic modification, conferred host resistance against DSS-induced colonic injury. Our findings serve as proof-of-concept that FMT may be an effective treatment for IBD, and that levels of DUSP6 expression may be useful in selecting appropriate microbiota donors and in stratifying patients/recipients. For example, individuals with lower DUSP6 expression in the colon or who express certain DUSP6 SNPs may be potential super donors for FMT in the treatment of IBD.

\section{Conclusions}

Our multi-omics and animal experiments have shown that a significant genetic alteration, such as the depletion of Dusp6, can be beneficial in enhancing intrinsic gut barrier integrity, elevating epithelial phosphoxidation, and maintaining the gut microbiota eubiosis necessary to protect against colitis. Dusp6 deficiency in mice provided early-onset protection from DSS-induced damage to the gut epithelium, indicating the potential benefits of microbiome-based strategies in prevention of IBD. 
We have only begun to recognize the implications of these findings on IBD therapeutics. The powerful interplay between novel treatment targets, such as DUSP6, pharmacological inhibitors, DUSP6-deficiency-derived microbiota therapeutics, and DUSP6-based precision medicine for FMT has significant potential for improving prevention and treatment of IBD, while enhancing both patient care and overall quality of life. 


\section{Methods}

For information on cell culture experiments, histological analysis, real-time RT-PCR, Western blotting, RNA-seq transcriptome profiling, phospho-proteomic profiling, 16S rRNA gene-based microbiome analysis, and cohousing and FMT experiments, see Supplemental material.

Mice. D6KO mice, originally obtained from the Jackson Laboratory, were transferred onto a C57BL/6J genetic background by backcrossing more than 10 generations. The D6KO mice and their WT littermates were weaned 3-4 weeks after birth and housed separately (3-4 mice/cage). Mice with the same genotype from different litters were housed together, as needed. All mice experienced a continuous 12-hour light/dark cycle, were fed an autoclaved chow diet, and were maintained under semi-specific pathogen-free conditions. When experiments were complete, the mice were euthanized via $\mathrm{CO}_{2}$ inhalation.

Induction of colonic epithelium damage by DSS. At 8-9 weeks of age, mice were treated with $2 \%(w / v)$ DSS (MW = 36,000-50,000; MP Biomedicals, Solon, $\mathrm{OH}, \mathrm{USA}$ ) through their drinking water for 9 days. The Disease Activity Index (DAI), ranging from 0 to 16, was determined for each mouse daily by scoring weight loss, stool consistency, and rectal bleeding of mice throughout DSS treatment, as described previously [66]. The scores were defined as follows: weight loss (no loss = $0 ;<5 \%=1 ; 5-10 \%=2 ; 10-20 \%=3 ;>20 \%=4$ ), stool consistency (normal $=0 ;$ loose stool = 2; diarrhea $=4$ ), and rectal bleeding (no blood $=0$; hemoccult positive $=1$; visual pellet bleeding $=2$; 
gross bleeding or blood around anus $=4$ ). On day 9, mice were euthanized, and the spleen and colon were harvested. The colon was flushed with phosphate-buffered saline (PBS, pH 7.4) and colon length was measured. Approximately $2 \mathrm{~cm}$ of the proximal colon (adjacent to the caecum) was removed and fixed with $4 \%$ formaldehyde for subsequent histological analysis. Approximately 0.5 $\mathrm{cm}$ of the distal colon was stored in RNAlater stabilization solution (Qiagen, Hilden, Germany) for subsequent RNA analysis.

Statistical analysis for non-omics data. Comparisons between two groups were performed using the Student's $t$-test. Comparisons of more than two data sets were performed using one-way analysis of variance followed by Fisher's least significant difference (LSD) multiple comparison test. Differences were considered significant when $P<0.05$. Data are expressed as mean \pm SEM. 


\section{Declarations}

Ethics approval and consent to participate. Animal experimentation protocols were approved by the Institutional Animal Care and Use Committee of the National Health Research Institutes, Taiwan; approval NHRI-IACUC-107046-A.

Consent for publication. Not applicable.

Competing interests. The authors declare that they have no competing interests.

Availability of data and materials. Raw sequencing data files have been uploaded to NCBI under the following BioProject ID: PRJNA589474 (SRA: SRP229874). The data that support the findings of this study are available, upon request, from C.-Y.K.

Author contributions. C.-S.C., H.-F. J., H.-C. H., H.-L.C., Y.-C.L., and C.-Y.K. designed the study. C.-S.C., C. H.-Y. C., C.-T.H., C.-M. L., J.-W.R., and Y.-T.T. performed experiments and analyzed the data. Y.-C.L., W.-H.Y., and C.-Y.K. performed bioinformatic analyses. T.-H.T. and Y.-W. S. provided D6KO mice. C.-S.C. and C.-Y.K. wrote the manuscript. C.-Y.K. performed overall supervision of the project.

Acknowledgments. The authors thank Dr. Wann-Neng Jane at the Cell Biology Core Lab, Electron Microscope Division, Institute of Plant and Microbial Biology, Academia Sinica, Taiwan, for technical assistance in use of the transmission electron microscope. We are grateful to Dr. Shau-Ku Huang at the National Institute of Environmental Health Sciences, NHRI, for critical reading and stimulating discussions during the preparation of the manuscript. We are grateful to the National Center for High-performance Computing for use of computers and facilities. We are grateful to Deng-Yu Jiang 
for assistance of Western blotting. We are also grateful to Loretta Collins of WriteScience, LLC for scientific editing and proofreading. This work was supported by the following grants: IM-108-PP-04 from NHRI and 106-2628-B-400-001-MY3, 106-2923-B-400-001-MY3, 108-2321-B-400-011-, 1092327-B-400-001-, 109-2320-B-400-008-MY3 from MOST. The funding agencies had no role in study design, data collection and analysis, decision to publish, or preparation of the manuscript. 


\section{References}

1. Konig J, Wells J, Cani PD, Garcia-Rodenas CL, MacDonald T, Mercenier A, et al. Human intestinal barrier function in health and disease. Clin Transl Gastroenterol. 2016;7:e196.

2. Catalioto RM, Maggi CA, Giuliani S. Intestinal epithelial barrier dysfunction in disease and possible therapeutical interventions. Curr Med Chem. 2011;18:398-426.

3. Shen L. Tight junctions on the move: molecular mechanisms for epithelial barrier regulation. Ann NY Acad Sci. 2012;1258:9-18.

4. Wells JM, Rossi O, Meijerink M, van Baarlen P. Epithelial crosstalk at the microbiota-mucosal interface. Proc Natl Acad Sci U S A. 2011;108 Suppl 1:4607-14.

5. Glover LE, Lee JS, Colgan SP. Oxygen metabolism and barrier regulation in the intestinal mucosa. J Clin Invest. 2016;126:3680-8.

6. Litvak Y, Byndloss MX, Baumler AJ. Colonocyte metabolism shapes the gut microbiota. Science. 2018;362:eaat9076.

7. Mohanan V, Nakata T, Desch AN, Levesque C, Boroughs A, Guzman G, et al. C1orf106 is a colitis risk gene that regulates stability of epithelial adherens junctions. Science. 2018;359:1161-6.

8. Anderson CA, Boucher G, Lees CW, Franke A, D'Amato M, Taylor KD, et al. Meta-analysis identifies 29 additional ulcerative colitis risk loci, increasing the number of confirmed associations to 47. Nat Genet. 2011;43:246-52.

9. Rivas MA, Beaudoin M, Gardet A, Stevens C, Sharma Y, Zhang CK, et al. Deep resequencing 
of GWAS loci identifies independent rare variants associated with inflammatory bowel disease. Nat Genet. 2011;43:1066-73.

10. Barrett JC, Lee JC, Lees CW, Prescott NJ, Anderson CA, Phillips A, et al. Genome-wide association study of ulcerative colitis identifies three new susceptibility loci, including the HNF4A region. Nat Genet. 2009;41:1330-4.

11. Silverberg MS, Cho JH, Rioux JD, McGovern DP, Wu J, Annese V, et al. Ulcerative colitisrisk loci on chromosomes 1 p36 and 12q15 found by genome-wide association study. Nat Genet. 2009;41:216-20.

12. Chang CS, Kao CY. Current understanding of the gut microbiota shaping mechanisms. J Biomed Sci. 2019;26:59.

13. Ulluwishewa D, Anderson RC, McNabb WC, Moughan PJ, Wells JM, Roy NC. Regulation of tight junction permeability by intestinal bacteria and dietary components. J Nutr. 2011;141:769-76.

14. Berry D, Reinisch W. Intestinal microbiota: a source of novel biomarkers in inflammatory bowel diseases? Best Pract Res Clin Gastroenterol. 2013;27:47-58.

15. Ni J, Wu GD, Albenberg L, Tomov VT. Gut microbiota and IBD: causation or correlation? Nat Rev Gastroenterol Hepatol. 2017;14:573-84.

16. Odenwald MA, Turner JR. The intestinal epithelial barrier: a therapeutic target? Nat Rev Gastroenterol Hepatol. 2017;14:9-21.

17. Viggiano D, laniro G, Vanella G, Bibbo S, Bruno G, Simeone G, et al. Gut barrier in health 
and disease: focus on childhood. Eur Rev Med Pharmacol Sci. 2015;19:1077-85.

18. Ruan JW, Statt S, Huang CT, Tsai YT, Kuo CC, Chan HL, et al. Dual-specificity phosphatase 6 deficiency regulates gut microbiome and transcriptome response against diet-induced obesity in mice. Nat Microbiol. 2016;2:16220.

19. Olsen J, Gerds TA, Seidelin JB, Csillag C, Bjerrum JT, Troelsen JT, et al. Diagnosis of ulcerative colitis before onset of inflammation by multivariate modeling of genome-wide gene expression data. Inflamm Bowel Dis. 2009;15:1032-8.

20. Smillie CS, Biton M, Ordovas-Montanes J, Sullivan KM, Burgin G, Graham DB, et al. Intraand inter-cellular rewiring of the human colon during ulcerative colitis. Cell. 2019;178:714-30 e22.

21. Cheung $\mathrm{CHY}$, Hsu CL, Chen KP, Chong ST, Wu CH, Huang HC, et al. MCM2-regulated functional networks in lung cancer by multi-dimensional proteomic approach. Sci Rep. 2017;7:13302.

22. Gericke B, Amiri M, Naim HY. The multiple roles of sucrase-isomaltase in the intestinal physiology. Mol Cell Pediatr. 2016;3:2.

23. Deng D, Yan N. GLUT, SGLT, and SWEET: Structural and mechanistic investigations of the glucose transporters. Protein Sci. 2016;25:546-58.

24. Schmitt CC, Aranias T, Viel T, Chateau D, Le Gall M, Waligora-Dupriet AJ, et al. Intestinal invalidation of the glucose transporter GLUT2 delays tissue distribution of glucose and reveals an unexpected role in gut homeostasis. Mol Metab. 2017;6:61-72. 
25. Schirmer M, Franzosa EA, Lloyd-Price J, Mclver LJ, Schwager R, Poon TW, et al. Dynamics of metatranscription in the inflammatory bowel disease gut microbiome. Nat Microbiol. 2018;3:337-46.

26. Hall AB, Yassour M, Sauk J, Garner A, Jiang X, Arthur T, et al. A novel Ruminococcus gnavus clade enriched in inflammatory bowel disease patients. Genome Med. 2017;9:103.

27. Zhou Y, Chen H, He H, Du Y, Hu J, Li Y, et al. Increased Enterococcus faecalis infection is associated with clinically active Crohn disease. Medicine (Baltimore). 2016;95:e5019.

28. Wragg P, La Ragione RM, Best A, Reichel R, Anjum MF, Mafura M, et al. Characterisation of Escherichia fergusonii isolates from farm animals using an Escherichia coli virulence gene array and tissue culture adherence assays. Res Vet Sci. 2009;86:27-35.

29. Arias CA, Murray BE. The rise of the Enterococcus: beyond vancomycin resistance. Nat Rev Microbiol. 2012;10:266-78.

30. Archambaud C, Derre-Bobillot A, Lapaque N, Rigottier-Gois L, Serror P. Intestinal translocation of enterococci requires a threshold level of enterococcal overgrowth in the lumen. Sci Rep. 2019;9:8926.

31. Fornefett J, Krause J, Klose K, Fingas F, Hassert R, Benga L, et al. Comparative analysis of humoral immune responses and pathologies of BALB/c and C57BL/6 wildtype mice experimentally infected with a highly virulent Rodentibacter pneumotropicus (Pasteurella pneumotropica) strain. BMC Microbiol. 2018;18:45.

32. Rigottier-Gois L. Dysbiosis in inflammatory bowel diseases: the oxygen hypothesis. ISME J. 
$2013 ; 7: 1256-61$.

33. Osaka T, Moriyama E, Arai S, Date Y, Yagi J, Kikuchi J, et al. Meta-Analysis of Fecal Microbiota and Metabolites in Experimental Colitic Mice during the Inflammatory and Healing Phases. Nutrients. 2017;9:1329.

34. Bertin S, Lozano-Ruiz B, Bachiller V, Garcia-Martinez I, Herdman S, Zapater P, et al. Dualspecificity phosphatase 6 regulates CD4+ T-cell functions and restrains spontaneous colitis in IL-10-deficient mice. Mucosal Immunol. 2015;8:505-15.

35. Kiesler P, Fuss IJ, Strober W. Experimental models of inflammatory bowel diseases. Cell Mol Gastroenterol Hepatol. 2015;1:154-70.

36. Sellon RK, Tonkonogy S, Schultz M, Dieleman LA, Grenther W, Balish E, et al. Resident enteric bacteria are necessary for development of spontaneous colitis and immune system activation in interleukin-10-deficient mice. Infect Immun. 1998;66:5224-31.

37. Beaudry K, Langlois MJ, Montagne A, Cagnol S, Carrier JC, Rivard N. Dual-specificity phosphatase 6 deletion protects the colonic epithelium against inflammation and promotes both proliferation and tumorigenesis. J Cell Physiol. 2019;234:6731-45.

38. Simren M, Barbara G, Flint HJ, Spiegel BM, Spiller RC, Vanner S, et al. Intestinal microbiota in functional bowel disorders: a Rome foundation report. Gut. 2013;62:159-76.

39. Henson MA, Phalak P. Microbiota dysbiosis in inflammatory bowel diseases: in silico investigation of the oxygen hypothesis. BMC Syst Biol. 2017;11:145.

40. Zhang W, Jiao L, Liu R, Zhang Y, Ji Q, Zhang H, et al. The effect of exposure to high altitude 
and low oxygen on intestinal microbial communities in mice. PLoS One. 2018;13:e0203701.

41. Chong CYL, Bloomfield FH, O'Sullivan JM. Factors Affecting Gastrointestinal Microbiome Development in Neonates. Nutrients. 2018;10:274.

42. Cresci GA, Bawden E. Gut microbiome: what we do and don't know. Nutr Clin Pract. $2015 ; 30: 734-46$.

43. Schaffer K, Taylor CT. The impact of hypoxia on bacterial infection. FEBS J. 2015;282:22606.

44. Gil-Marques ML, Pachon-Ibanez ME, Pachon J, Smani Y. Effect of hypoxia on the pathogenesis of Acinetobacter baumannii and Pseudomonas aeruginosa in vitro and in murine experimental models of infection. Infect Immun. 2018;86:e00543-18.

45. Kelly CJ, Glover LE, Campbell EL, Kominsky DJ, Ehrentraut SF, Bowers BE, et al. Fundamental role for HIF-1alpha in constitutive expression of human beta defensin-1. Mucosal Immunol. 2013;6:1110-8.

46. Colgan SP, Taylor CT. Hypoxia: an alarm signal during intestinal inflammation. Nat Rev Gastroenterol Hepatol. 2010;7:281-7.

47. Hirota SA, Fines K, Ng J, Traboulsi D, Lee J, Ihara E, et al. Hypoxia-inducible factor signaling provides protection in Clostridium difficile-induced intestinal injury. Gastroenterology. 2010;139:259-69 e3.

48. Karhausen J, Furuta GT, Tomaszewski JE, Johnson RS, Colgan SP, Haase VH. Epithelial hypoxia-inducible factor-1 is protective in murine experimental colitis. J Clin Invest. 
2004;114:1098-106.

49. Bermudez O, Jouandin P, Rottier J, Bourcier C, Pages G, Gimond C. Post-transcriptional regulation of the DUSP6/MKP-3 phosphatase by MEK/ERK signaling and hypoxia. J Cell Physiol. 2011;226:276-84.

50. Lee SH. Intestinal permeability regulation by tight junction: implication on inflammatory bowel diseases. Intest Res. 2015;13:11-8.

51. Watson AJ. Claudins and barrier dysfunction in intestinal inflammation: cause or consequence? Gut. 2015;64:1501-2.

52. Laukoetter MG, Nava P, Lee WY, Severson EA, Capaldo CT, Babbin BA, et al. JAM-A regulates permeability and inflammation in the intestine in vivo. J Exp Med. 2007;204:306776.

53. Chen P, Bakke D, Kolodziej L, Lodolce J, Weber CR, Boone DL, et al. Antrum Mucosal Protein-18 peptide targets tight junctions to protect and heal barrier structure and function in models of inflammatory bowel disease. Inflamm Bowel Dis. 2015;21:2393-402.

54. Talavera MM, Jin Y, Zmuda EJ, Frick J, McBride KL, Nelin LD, et al. Single nucleotide polymorphisms in the dual specificity phosphatase genes and risk of necrotizing enterocolitis in premature infant. J Neonatal Perinatal Med. 2019;Pre-press:1-8.

55. Feng $\mathrm{B}, \mathrm{He} \mathrm{Q}, \mathrm{Xu} \mathrm{H}$. FOXO1-dependent up-regulation of MAP kinase phosphatase 3 (MKP3) mediates glucocorticoid-induced hepatic lipid accumulation in mice. Mol Cell Endocrinol. 2014;393:46-55. 
56. Sawada N. Tight junction-related human diseases. Pathol Int. 2013;63:1-12.

57. Hsu SF, Lee YB, Lee YC, Chung AL, Apaya MK, Shyur LF, et al. Dual specificity phosphatase DUSP6 promotes endothelial inflammation through inducible expression of ICAM-1. FEBS J. 2018;285:1593-610.

58. Wu QN, Liao YF, Lu YX, Wang Y, Lu JH, Zeng ZL, et al. Pharmacological inhibition of DUSP6 suppresses gastric cancer growth and metastasis and overcomes cisplatin resistance. Cancer Lett. 2018;412:243-55.

59. VanDussen KL, Stojmirovic A, Li K, Liu TC, Kimes PK, Muegge BD, et al. Abnormal small intestinal epithelial microvilli in patients with Crohn's disease. Gastroenterology. 2018;155:815-28.

60. Grimm-Gunter EM, Revenu C, Ramos S, Hurbain I, Smyth N, Ferrary E, et al. Plastin 1 binds to keratin and is required for terminal web assembly in the intestinal epithelium. Mol Biol Cell. 2009;20:2549-62.

61. Paramsothy S, Kamm MA, Kaakoush NO, Walsh AJ, van den Bogaerde J, Samuel D, et al. Multidonor intensive faecal microbiota transplantation for active ulcerative colitis: a randomised placebo-controlled trial. Lancet. 2017;389:1218-28.

62. Moayyedi P, Surette MG, Kim PT, Libertucci J, Wolfe M, Onischi C, et al. Fecal microbiota transplantation induces remission in patients with active ulcerative colitis in a randomized controlled trial. Gastroenterology. 2015;149:102-9 e6.

63. Rossen NG, Fuentes S, van der Spek MJ, Tijssen JG, Hartman JH, Duflou A, et al. Findings 
from a randomized controlled trial of fecal transplantation for patients with ulcerative colitis.

Gastroenterology. 2015;149:110-8 e4.

64. Cui B, Feng Q, Wang H, Wang M, Peng Z, Li P, et al. Fecal microbiota transplantation through mid-gut for refractory Crohn's disease: safety, feasibility, and efficacy trial results. J Gastroenterol Hepatol. 2015;30:51-8.

65. Sokol H, Landman C, Seksik P, Berard L, Montil M, Nion-Larmurier I, et al. Fecal microbiota transplantation in crohn's disease: a pilot randomized, single-blind, shamcontrolled trial. United European Gastroenterology J. 2018;6:A249.

66. Kim JJ, Shajib MS, Manocha MM, Khan WI. Investigating intestinal inflammation in DSSinduced model of IBD. J Vis Exp. 2012:e3678. 


\section{Figures legends}

\section{Figure 1}

Dusp6 gene deficiency mitigated DSS-induced colitis. (A) DUSP6 expression levels in normal controls and UC patients (NCBI dataset GSE9452; normal: $n=5$; UC-inflamed: $n=8$; UC-noninflamed: $n=13$; each dot represents one microarray probeset for each group, with a total of 3 probesets mapped to DUSP6 in one microarray chip). ${ }^{* *} P<0.01$, Fisher's LSD test. (B, C, D) Composition of cells with diverse levels of DUSP6 mRNA expression, as defined by the number of unique molecular identifiers (UMIs), in epithelial, immune, or stromal cells from colonic biopsies of healthy subjects and inflamed and non-inflamed tissues of UC patients (Single Cell Portal: SCP259). ${ }^{* * *} P<0.001$, Mann-Whitney $U$ test. (E) Immunohistochemical staining of DUSP6 protein (brown) in colonic tissue from C57BL/6J mice after 9 days of treatment with water or water plus DSS (indicated as DSS throughout). (F) Daily records of changes of body weight of wild-type (WT) and Dusp6 gene knockout $(\mathrm{D} 6 \mathrm{KO})$ mice during 9 days of treatment with water or DSS. ${ }^{*} P<0.05$ and ${ }^{* *} P<0.001$, Fisher's LSD test. (G) The weights of WT and D6KO mice with or without DSS treatment. * $P<0.05$ and ${ }^{* * *} P<0.001$, Fisher's LSD test. (H) Daily disease activity index scores of WT and D6KO mice during 9 days of treatment with water or DSS. ${ }^{* *} P<0.001$, Fisher's LSD test. Number of mice per group in F-H: WT-water, $n=6$; D6KO-water, $n=6$; WT-DSS, $n=7$; D6KO-DSS, $n=7$. (I, J) Representative colon tissue and colon length in WT and D6KO mice after water or DSS treatment for 9 days. ${ }^{*} P<0.05$ and ${ }^{* * *} P<0.001$, Fisher's LSD test. Number of mice per group: WT-water, $n=$ 10; D6KO-water, $n=12$; WT-DSS, $n=11$; D6KO-DSS, $n=15$. (K) Representative H\&E stained 
transverse sections of colon tissue (top row) and colonic epithelium (bottom row; scale bar $=50 \mu \mathrm{m}$ )

from WT and D6KO mice after 9 days of treatment with water or DSS. (L) Representative Alcian blue stained transverse sections of colon tissue (top row; scale bar $=500 \mu \mathrm{m}$ ) and colonic epithelium (bottom row; scale bar $=50 \mu \mathrm{m}$ ) from WT and D6KO mice after 9 days of treatment with water or DSS. D6KO, Dusp6 gene knockout mice; DSS, dextran sulfate sodium; H\&E, hematoxylin and eosin; UC, ulcerative colitis; WT, wild-type mice.

\section{Figure 2}

\section{Dusp6 deficiency led to increased expression of tight junction proteins and elongated} microvilli in mouse colon tissue. (A) qRT-PCR analysis of tight junction-associated genes in the colonic tissues of WT and D6KO mice after 9 days of treatment with water or water plus DSS (indicated as DSS throughout). ${ }^{*} P<0.05,{ }^{* *} P<0.01$, and ${ }^{* \star *} P<0.001$, Fisher's LSD test. Number of mice per group: WT-water, $n=10$; D6KO-water, $n=12$; WT-DSS, $n=11$; D6KO-DSS, $n=15$. (B) Immunofluorescence staining of TJP1, TJP2, and TJP3 (green) in colon tissue from WT and D6KO mice treated with water or DSS for 9 days. Images of the colonic epithelium along luminal and crypt axes were captured by confocal microscopy. Cell nuclei were stained with DAPI (blue). (C) Ultrastructural changes in the tight junctions and microvilli of the colonic epithelium in WT and D6KO mice via transmission electron microscopy. The tight junction complexes are indicated by yellow arrows. Scale bar $=200 \mathrm{~nm}$. (D) Quantitative analysis of colonic epithelial cell microvilli length in WT and D6KO mice. ${ }^{* \star *} P<0.001$, Student's $t$-test. Number of microvilli per group: WT, $n=66$; D6KO, $n$ 
= 62. (E) qRT-PCR analysis of microvilli-associated genes in the colonic tissue of WT and D6KO mice after 9 days of treatment with water or DSS. ${ }^{*} P<0.05,{ }^{* *} P<0.01$, and ${ }^{* * *} P<0.001$, Fisher's LSD test. Number of mice per group: WT-water, $n=10$; D6KO-water, $n=12$; WT-DSS, $n=11$; D6KODSS, $n=15$. D6KO, Dusp6 knockout mice; DSS, dextran sulfate sodium; WT, wild-type mice.

\section{Figure 3}

\section{DUSP6 knockout altered the transcriptome and phospho-proteome in human gut epithelial}

Caco-2 cells. (A) The heatmap of DEGs from RNA-seq analysis performed in WT and DUSP6 gene knockout (DUSP6 KO) Caco-2 cells after 21 days in culture ( $n=5$ in each group). The color coding indicates to relative gene expression level based on Z-score transformation. (B) Top 10 FunRich biological pathways of the transcriptome that were significantly up-regulated or down-regulated in DUSP6 KO compared to WT cells. (C) Top 20 FunRich biological pathways of the phosphoproteome that were significantly altered in DUSP6 KO compared to WT cells. (D) Intersection of upregulated transcriptomic and phospho-proteomic datasets shows the enriched biological pathways that could be modulated by DUSP6 deficiency. (E) qRT-PCR analysis of tight junction protein genes in WT and DUSP6 KO cells after 21 days in culture. ${ }^{*} P<0.05,{ }^{* *} P<0.01$, and ${ }^{* \star *} P<0.001$, Student's $t$-test; $n=5$ in each group. (F) Western blotting of DUSP6, TJP1, TJP2, and TJP3 protein from WT and DUSP6 KO cells after 21 days in culture. (G) Immunofluorescent staining of TJP1, TJP2, and TJP3 proteins in WT and DUSP6 KO cells after 7 days in culture. (H) qRT-PCR analysis of microvilli- 
associated genes in WT and DUSP6 KO cells after 21 days in culture. ${ }^{* \star} P<0.01$ and ${ }^{* *} P<0.001$, Student's $t$-test; $n=5$ in each group.

\section{Figure 4}

DUSP6 knockout elevated mitochondrial oxidation and attenuated glycolysis in Caco-2 cells.

(A) mRNA levels of hypoxia-associated genes in WT and DUSP6 KO cells after 21 days in culture. ${ }^{* *} P<0.01$ and ${ }^{* *} P<0.001$, Student's $t$-test; $n=5$ in each group. (B) Western blotting of HIF1A protein from WT and DUSP6 KO cells after 21 days in culture. $\beta$-tubulin protein was used as a loading control. (C) Real-time oxygen consumption rate (OCR) values from WT $(n=10)$ and DUSP6 KO $(n$ $=11)$ cells in response to the addition of oligomycin (Oli), carbonyl cyanide-ptrifluoromethoxyphenylhydrazone (FCCP) and rotenone plus antimycin A (R/A). (D) Statistical analysis of non-mitochondrial respiration, basal respiration, ATP production, proton leak, maximal respiration, and spare capacity based on oxygen consumption rate values. ${ }^{*} P<0.05,{ }^{* *} P<0.01$, and ${ }^{* * *} P<0.001$, Student's $t$-test. (E) Color change of phenol red-containing medium after incubation with WT and DUSP6 KO cells for 3 days. Image was taken on day 21 of cell culture. (F) Plot of basal OCR versus extracellular acidification rates (ECARs) of WT $(n=10)$ and DUSP6 KO $(n=11)$ cells in glucose-containing medium. (G) Real-time ECARs of WT $(n=10)$ and DUSP6 KO $(n=11)$ cells in response to the addition of glucose (Glu), oligomycin (Oli), and 2-deoxy-D-glucose (2-DG). (H) Statistical analysis of non-glycolytic acidification, glycolysis, glycolytic capacity, and glycolytic reserve based on ECAR values. ${ }^{* *} P<0.001$, Student's $t$-test. (I) qRT-PCR analysis of glucose 
uptake genes in WT and DUSP6 KO cells after 21 days in culture. ${ }^{* *} P<0.001$, Student's $t$-test; $n=$ 5 in each group. (J) Glucose utilization by WT and DUSP6 KO Caco-2 cells via evaluation of cellular ATP levels after supplementation with glucose for $4 \mathrm{hrs.}{ }^{* \star} P<0.01$, Student's $t$-test; $n=5$ in each group. DUSP6 KO, DUSP6 gene knockout Caco-2 cells; ECAR, extracellular acidification rate; OCR, oxygen consumption rate; WT, wild-type Caco-2 cells.

\section{Figure 5}

\section{Comparison of fecal microbiota derived from WT and D6KO mice before and after DSS}

treatment. (A) Phylum-level relative abundance of fecal microbiota of IBD and non-IBD subjects reanalyzed from the HMP dataset (IBD, $n=57$; non-IBD, $n=320$ ). (B, C) Composition of fecal microbiota, at phylum and family levels, from WT and D6KO mice before or after 9 days of DSS treatment. (D) BugBase analysis of the proportion of aerobes, facultative anaerobes, and obligate anaerobes in mouse gut. (E) Cladograms generated from LEfSe analysis showing the microbial clades with the greatest differences in abundance in microbiota from WT (green) or D6KO (red) mice after DSS treatment. (F) LDA scores of microbial clades differing in abundance between WT and D6KO mice after DSS treatment (with LDA score $>2$ and significance of $\alpha<0.05$, determined by Kruskal-Wallis test). (G) Comparison of changes in bacterial species in WT and D6KO mice after DSS treatment. Values are means for each group. Number of mice per group: WT-water, $n=48$ and D6KO-water, $n=51$; WT-DSS, $n=22$ and D6KO-DSS, $n=28$. D6KO, Dusp6 gene knockout mice; 
DSS, dextran sulfate sodium; HMP, human microbiome project; IBD, inflammatory bowel disease;

LDA, linear discriminant analysis; WT, wild-type mice.

\section{Figure 6}

Cohousing of WT mice with D6KO mice mitigated DSS-induced colitis. (A) Schematic of the cohousing experiment. Two D6KO mice and two WT littermates were housed together for 4 weeks, followed by 9 days of treatment with DSS. (B, C) Daily weight change and disease activity index scores for WT and D6KO mice, with or without cohousing, during 9 days of treatment with water or water plus DSS (indicated as DSS throughout). ${ }^{*} P<0.05$, ${ }^{* *} P<0.01$, and ${ }^{* * *} P<0.001$, Fisher's LSD test. (D) Colon length in WT and D6KO mice, with or without cohousing, after 9 days of treatment with DSS. ${ }^{*} P<0.05$ and ${ }^{* * *} P<0.001$, Fisher's LSD test. Number of mice per group in B-D: Noncohoused WT-water, $n=13$; Non-cohoused D6KO-water, $n=14$; Non-cohoused WT-DSS, $n=15$; Non-cohoused D6KO-DSS, $n=15$; Cohoused WT-water, $n=7$; Cohoused D6KO-water, $n=8$; Cohoused WT-DSS, $n=7$; Cohoused D6KO-DSS, $n=9$. (E) Representative H\&E (top row) or Alcian blue (bottom row) stained transverse sections of colon tissue from the WT and D6KO mice, with or without co-housing, after 9 days of DSS treatment. (F) Representative images of colonic epithelium stained with H\&E (top row) or Alcian blue (bottom row). (G, H) Composition of the fecal microbiota of DSS-treated WT and D6KO mice, with or without cohousing, at the phylum and family levels. (I) The proportion of aerobes, facultative anaerobes and obligate anaerobes in mouse gut analyzed via BugBase. Number of mice per group in G-I: Non-cohoused WT-DSS, $n=22$; Cohoused WT-DSS, 
$n=8$; Non-cohoused D6KO-DSS, $n=28$; Cohoused D6KO-DSS, $n=8$. D6KO, Dusp6 gene knockout mice; DSS, dextran sulfate sodium; H\&E, hematoxylin and eosin; WT, wild-type mice.

\section{Figure 7}

Fecal microbiota derived from D6KO mice mitigated DSS-induced colitis. (A) Schematic of the FMT experiment. The fecal microbiota were collected from non-cohoused WT or D6KO mice within 30 min and transferred by oral gavage into germ-free mice three times per week. After the seventh FMT, the mice were rested for 3 days and then treated with DSS for 9 days. (B) Daily change in body weight was recorded for germ-free mice transplanted with fecal microbiota from WT or D6KO mice during 9 days of treatment with water or water plus DSS (indicated as DSS throughout). ${ }^{*} P<0.05,{ }^{* *} P<0.01$, and ${ }^{* *} P<0.001$, Fisher's LSD test. (C) Daily disease activity index scores of germ-free mice transplanted with gut microbiota from WT or D6KO mice during 9 days of treatment with water or DSS. ${ }^{* *} P<0.01$ and ${ }^{* * *} P<0.001$, Fisher's LSD test. Number of mice per group in B-C: Donor:WT-water, $n=4$; Donor:D6KO-water, $n=4$; Donor:WT-DSS, $n=5$; Donor:D6KO-DSS, $n=7$. (D) Colon length in germ-free mice with gut microbiota from WT or D6KO mice during 9 days of treatment with water or DSS. ${ }^{* \star *} P<0.001$, Fisher's LSD test. Number of mice per group: Donor:WTwater, $n=7$; Donor:D6KO-water, $n=7$; Donor:WT-DSS, $n=9$; Donor:D6KO-DSS, $n=10$.

Representative H\&E (top row) or Alcian blue (bottom row) stained transverse sections of colon tissue from germ-free mice with fecal microbiota from WT or D6KO mice after 9 days of treatment with DSS. (F) Representative images of colonic epithelium stained with H\&E (top row) or Alcian blue 
(bottom row). (G, H) qRT-PCR analysis of colonic tissues from Donor:WT and Donor:D6KO mice for expression of tight junction-associated genes and inflammatory cytokine genes after 9 days of treatment with water or DSS. ${ }^{*} P<0.05,{ }^{* *} P<0.01$, and ${ }^{* * *} P<0.001$, Fisher's LSD test. Number of mice per group in G-H: Donor:WT-water, $n=7$; Donor:D6KO-water, $n=7$; Donor:WT-DSS, $n=8$; Donor:D6KO-DSS, $n=9$. D6KO, Dusp6 gene knockout mice; Donor:WT, germ-free mice with fecal microbiota transplantation from wild-type mice; Donor:D6KO, germ-free mice with fecal microbiota transplantation from Dusp6 knockout mice; DSS, dextran sulfate sodium; FMT, fecal microbiota transplantation; H\&E, hematoxylin and eosin; WT, wild-type mice.

\section{Figure 8}

Dusp6 deficiency protects against DSS-induced colitis in mice. Dusp6 deficiency in mice results in induction of tight junction protein and microvilli-associated gene expression, which strengthens the connections between gut epithelial cells and extends the distance between microbes and the cytoplasm of enterocytes, thereby enhancing barrier integrity. Dusp6 deficiency also alters the cellular metabolic machinery, resulting in elevation of mitochondrial phosphorylation and reduction of glucose utilization. These changes maintain the gut lumen in an anaerobic state, which is an environment that promotes expansion of obligatory bacteria and limits the abundance of facultative bacteria and/or pathobionts. Therefore, Dusp6-deficient mice are resistant to DSSinduced epithelial damage and associated colitis. 
Figures

Figure 1
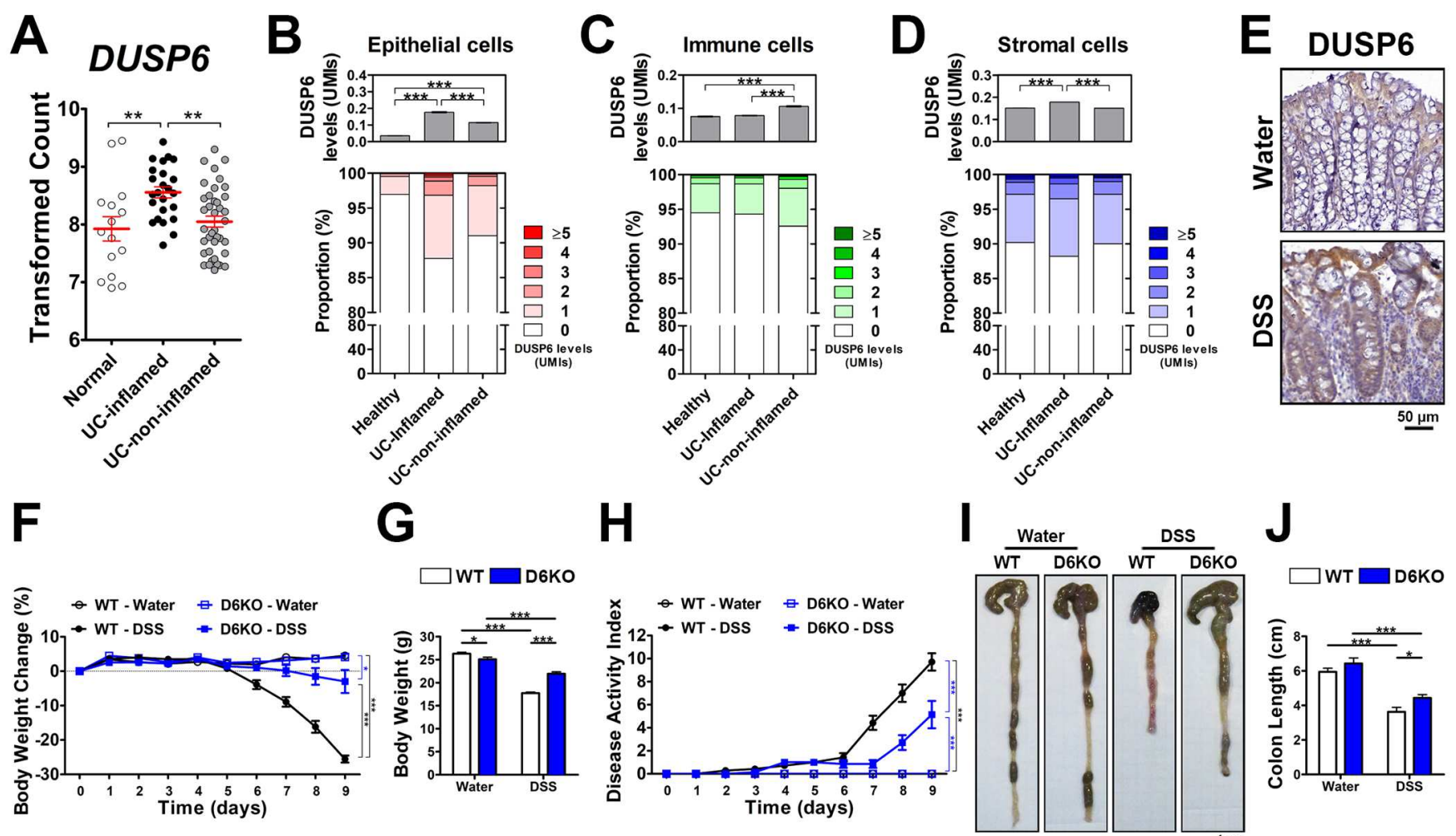

H
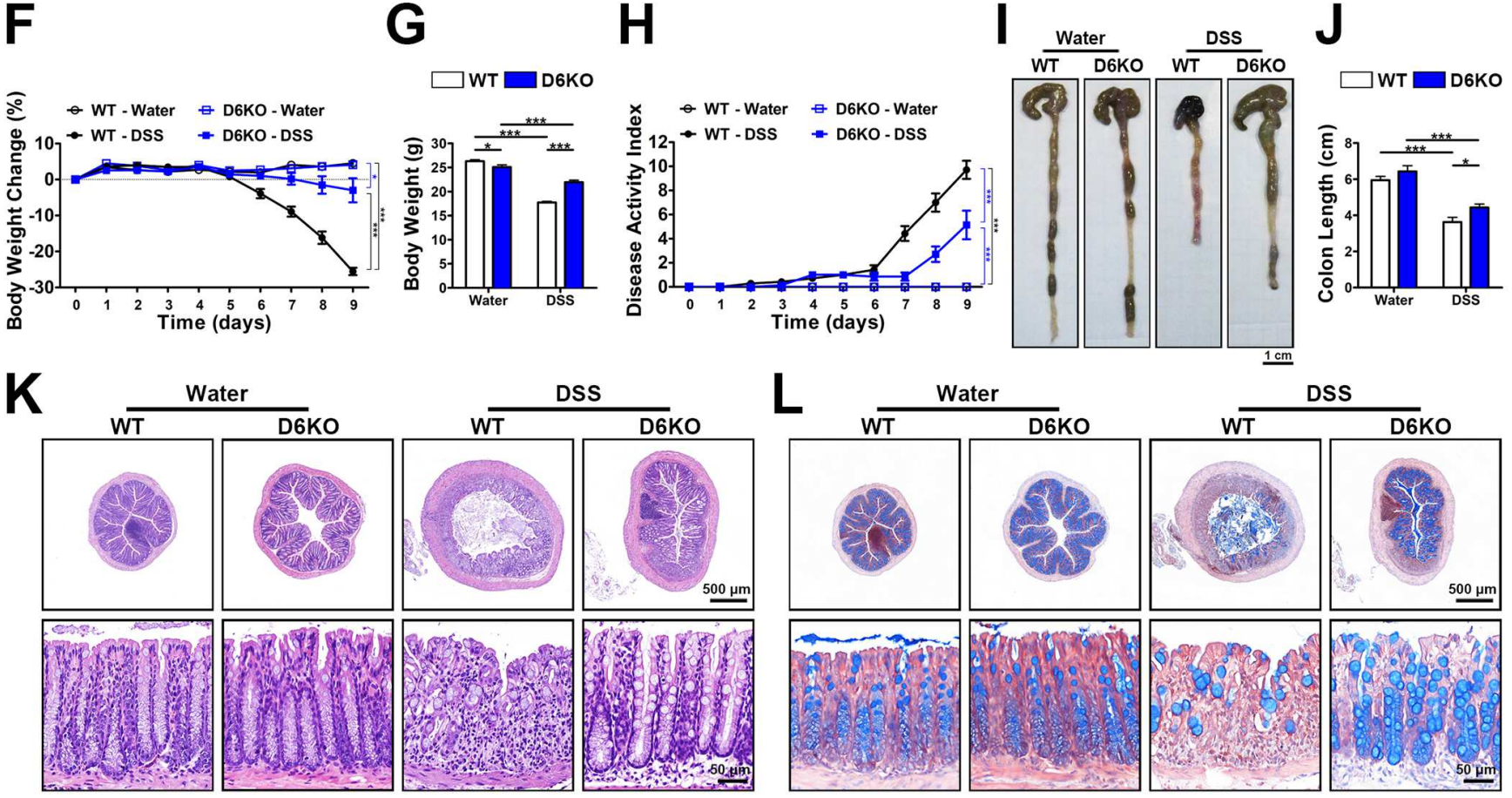
Figure 2

A
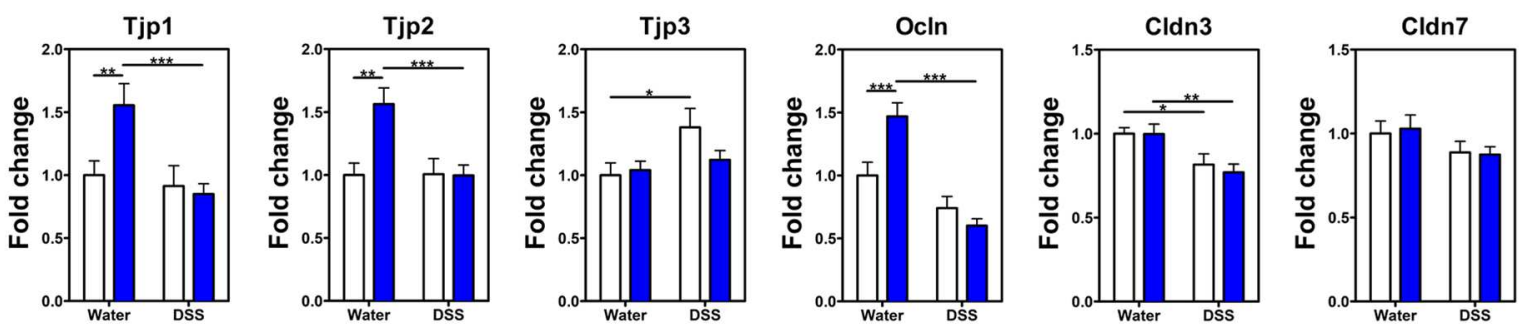

B

TJP1/DAPI

TJP2/DAPI

TJP3/DAPI
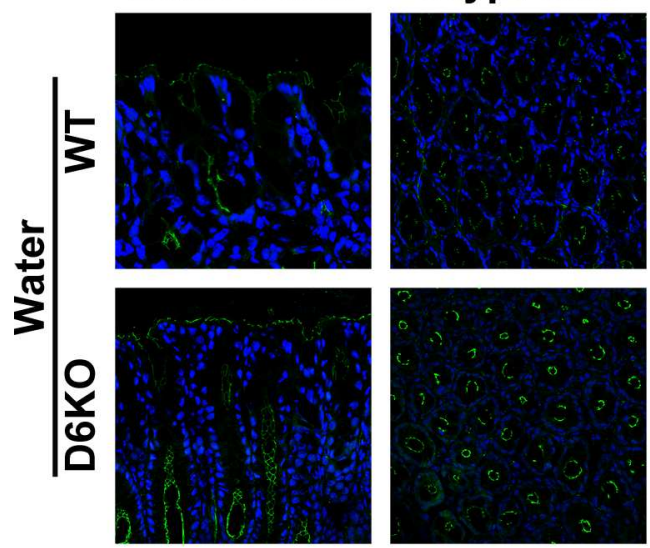

Lumen-axis Crypt-axis

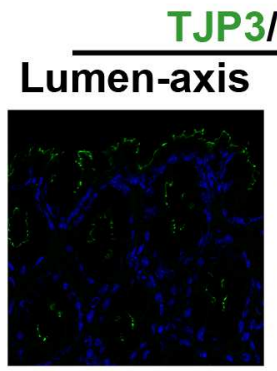

Crypt-axis
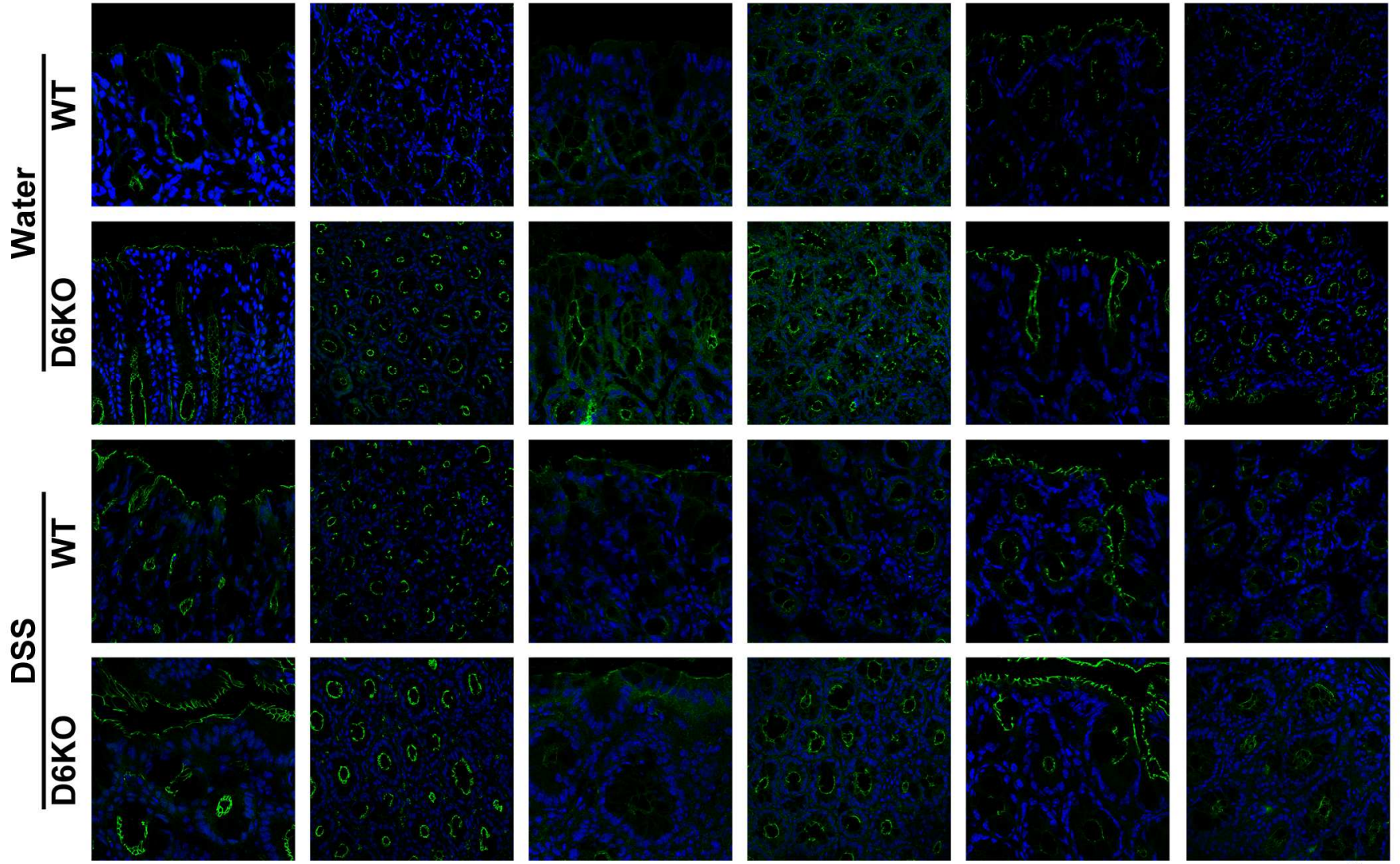

C
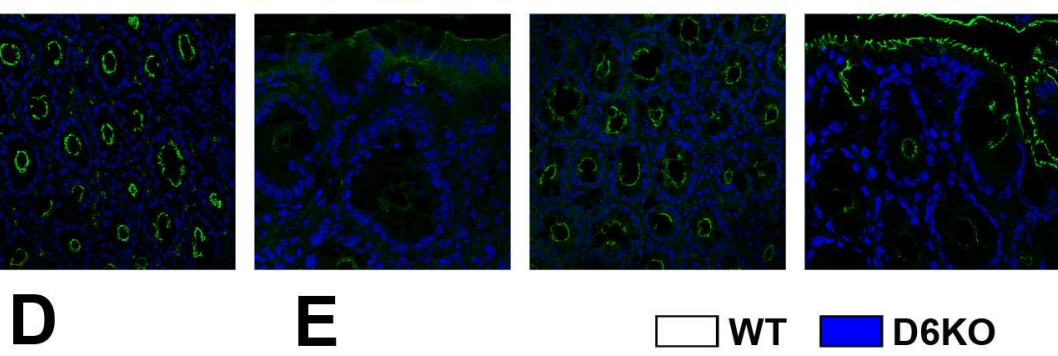

E

$\square$ WT $\square$ D6KO
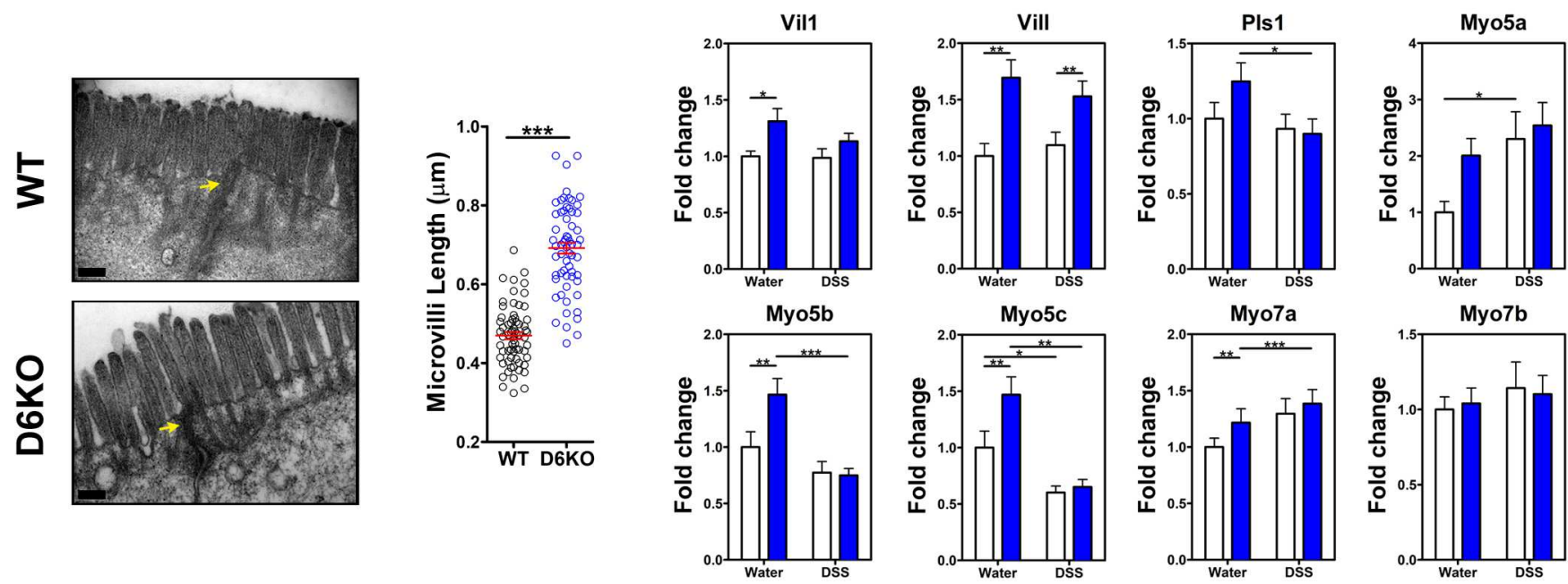
Figure 3

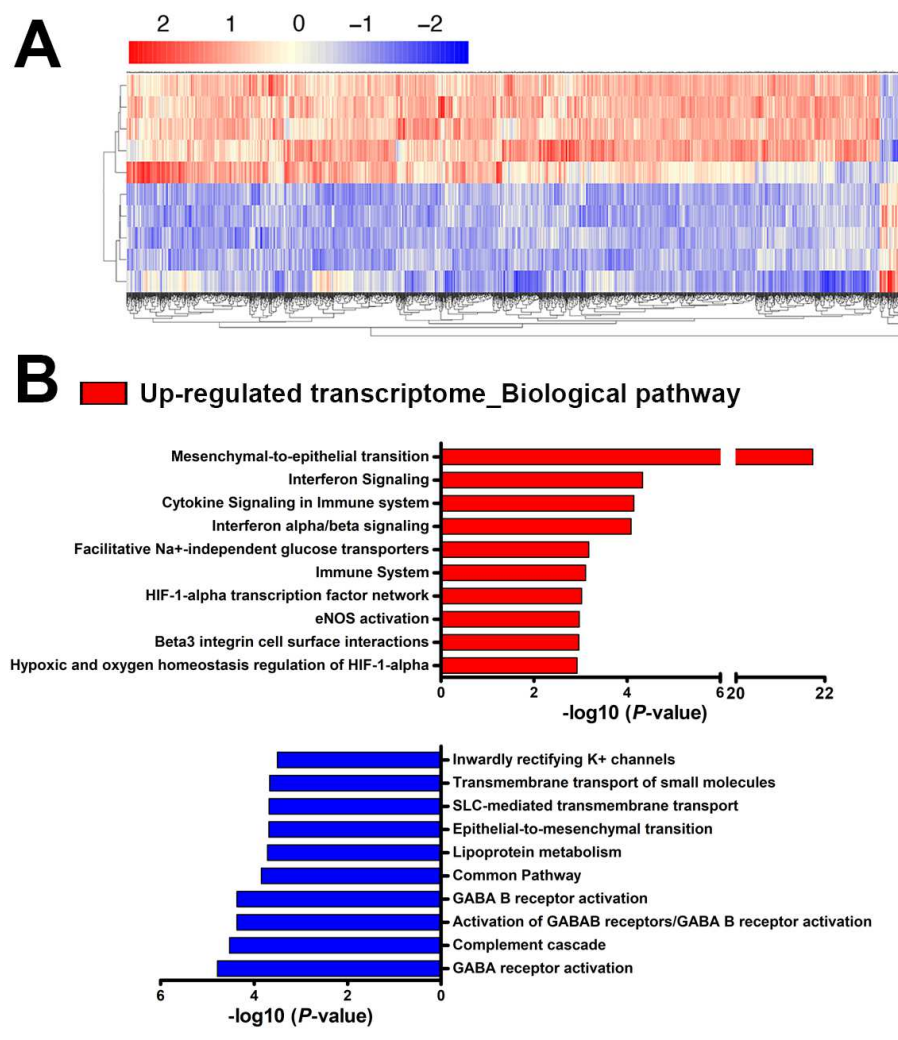

C

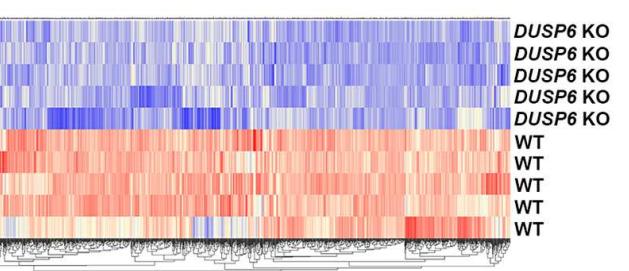

B

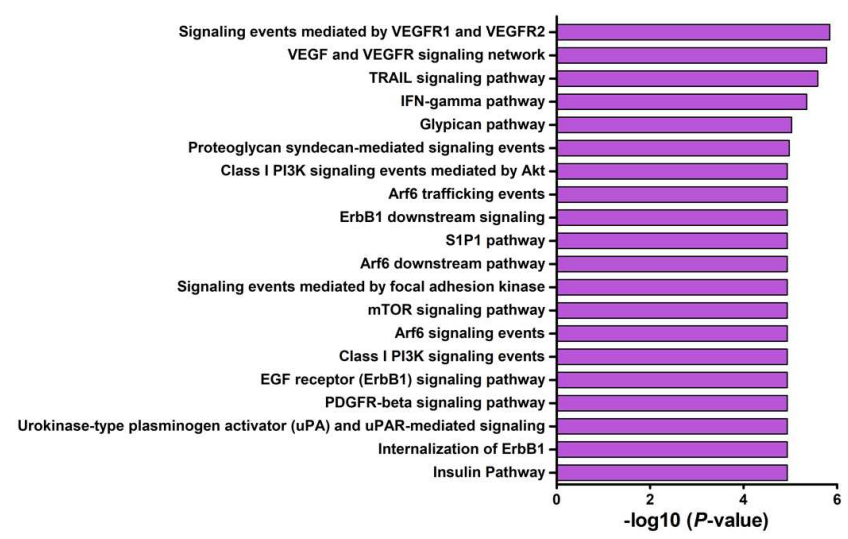

$\mathbf{D}$

Down-regulated transcriptome_Biological pathway
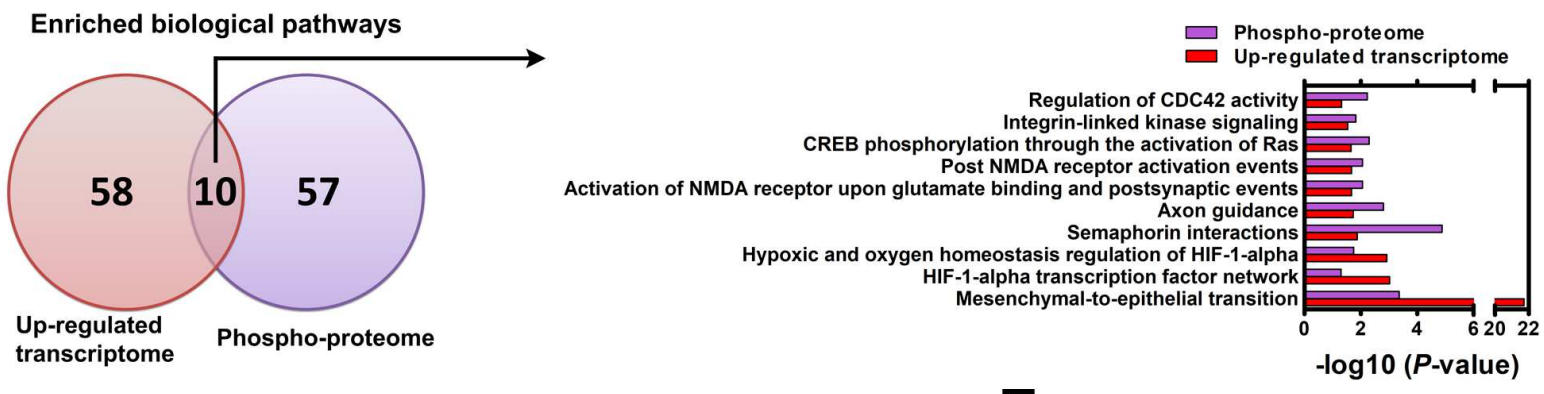

E

TJP1
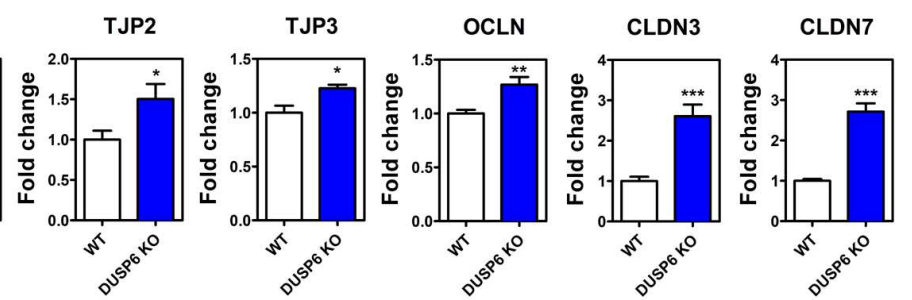

$F$

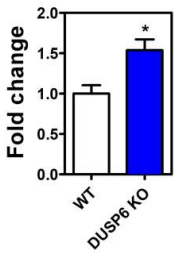

G

TJP1

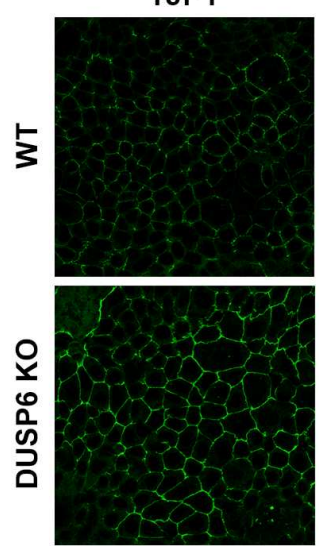

TJP2

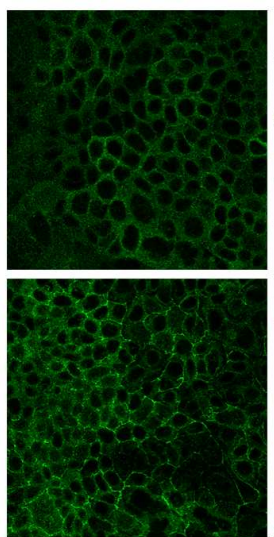

TJP3

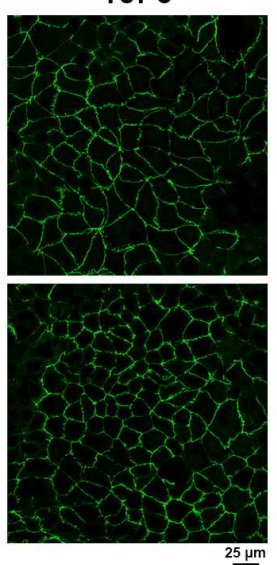

H
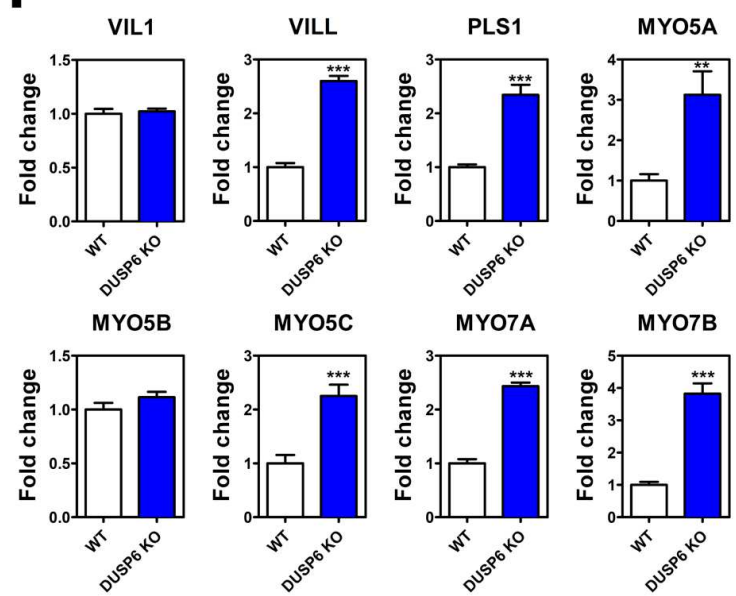
Figure 4

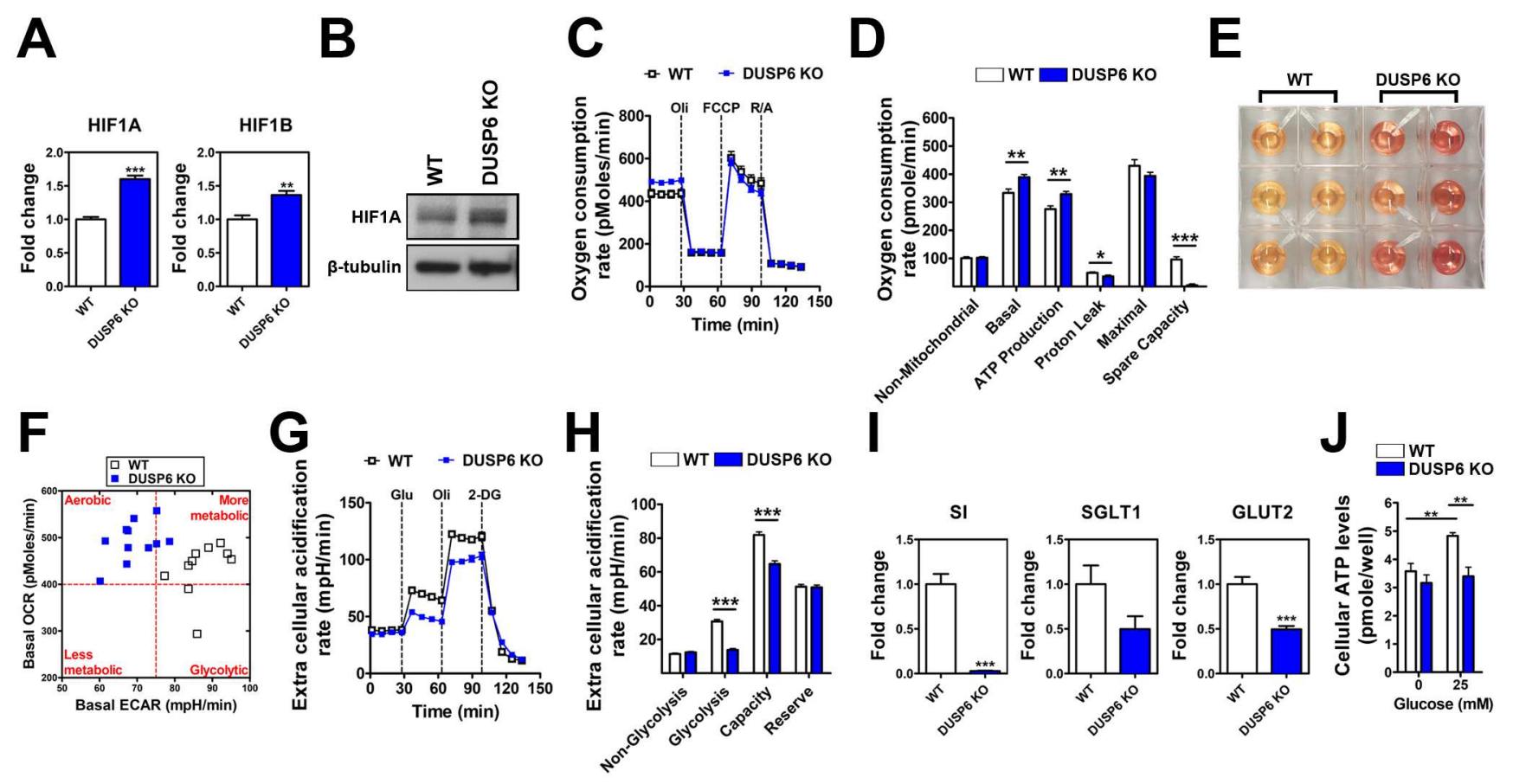


Figure 5

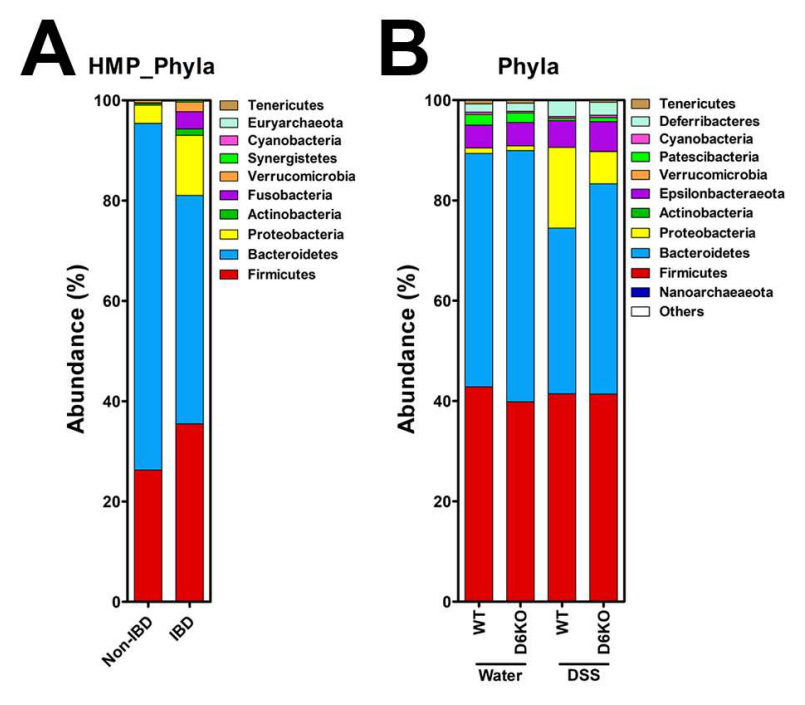

E
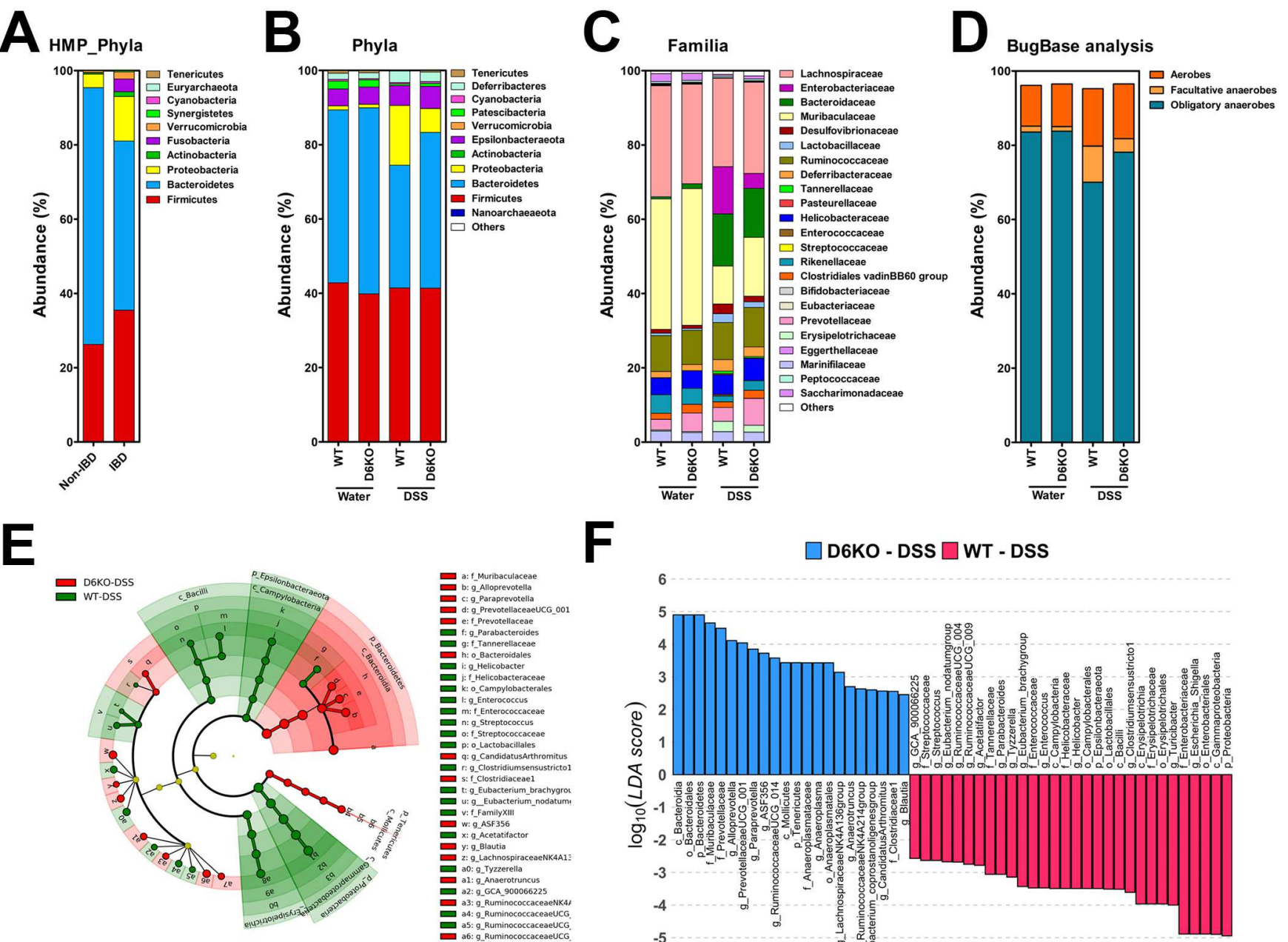

F

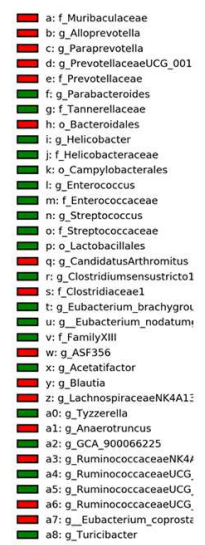

G
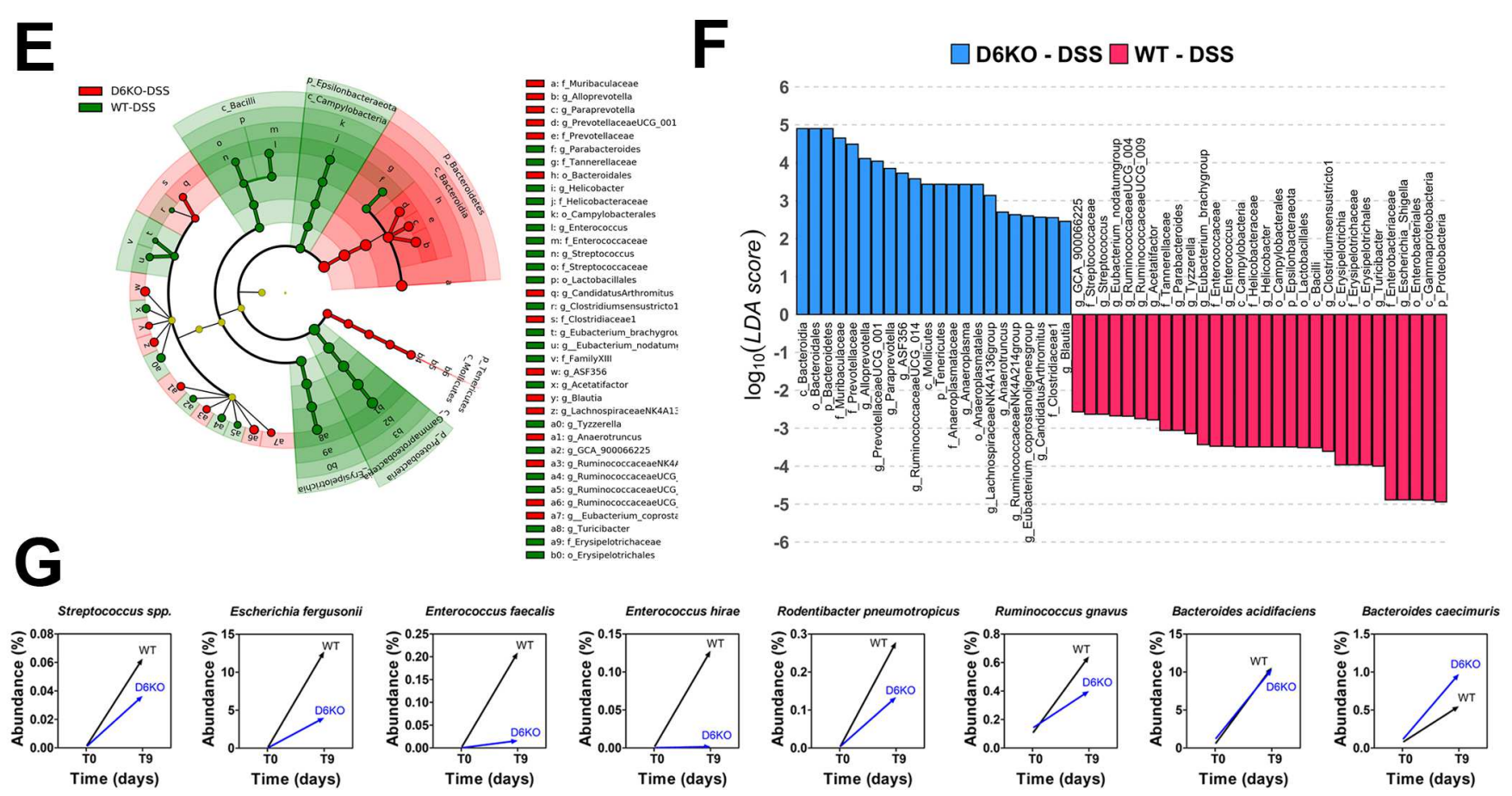
Figure 6

A $_{\text {Week }-4}$

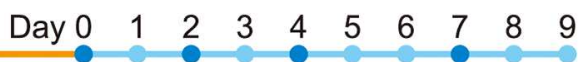

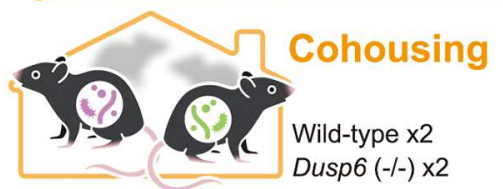

DSS or normal drinking water
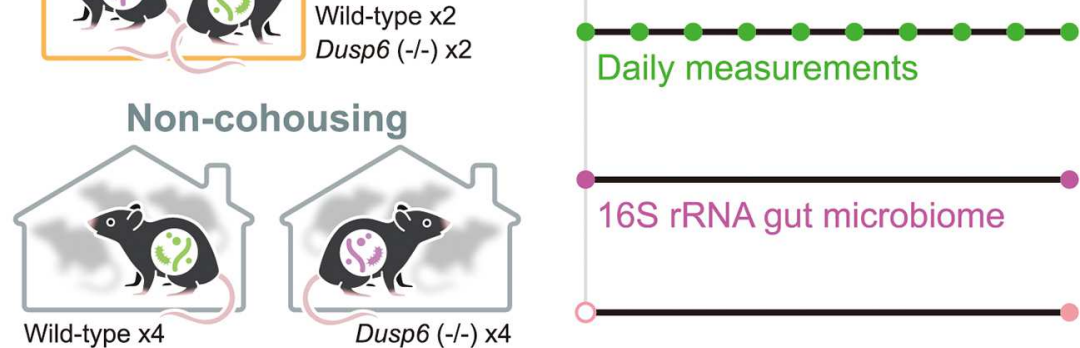

loss

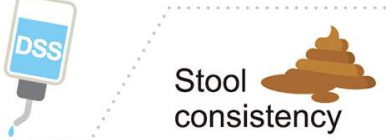

$$
\text { Wild-type } x 4
$$

Dusp6 (-/-) x4

B
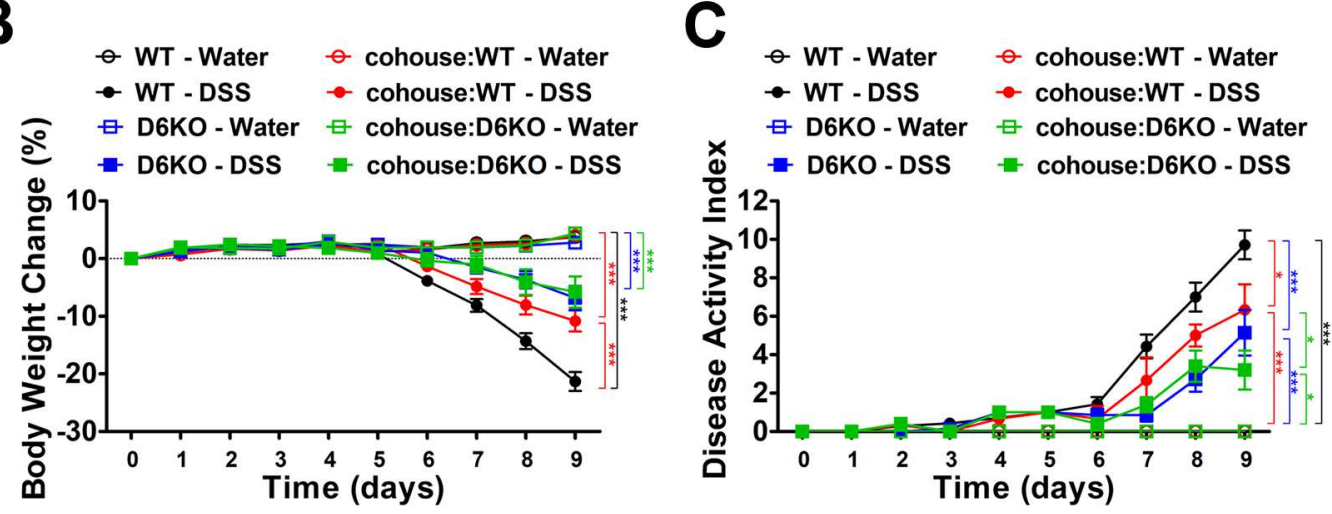

consistency

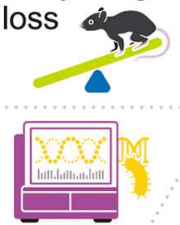

bleeding

Colonic
morphology

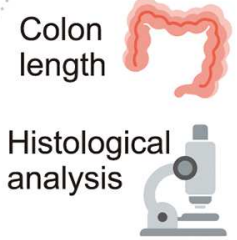

D $\square$ wT-Dss

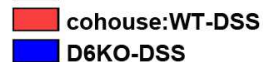

D6KO-DSS

$\square$ cohouse:D6KO-DSs

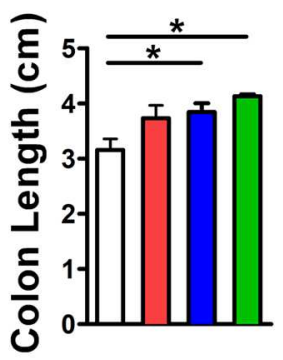

E

DSS

F
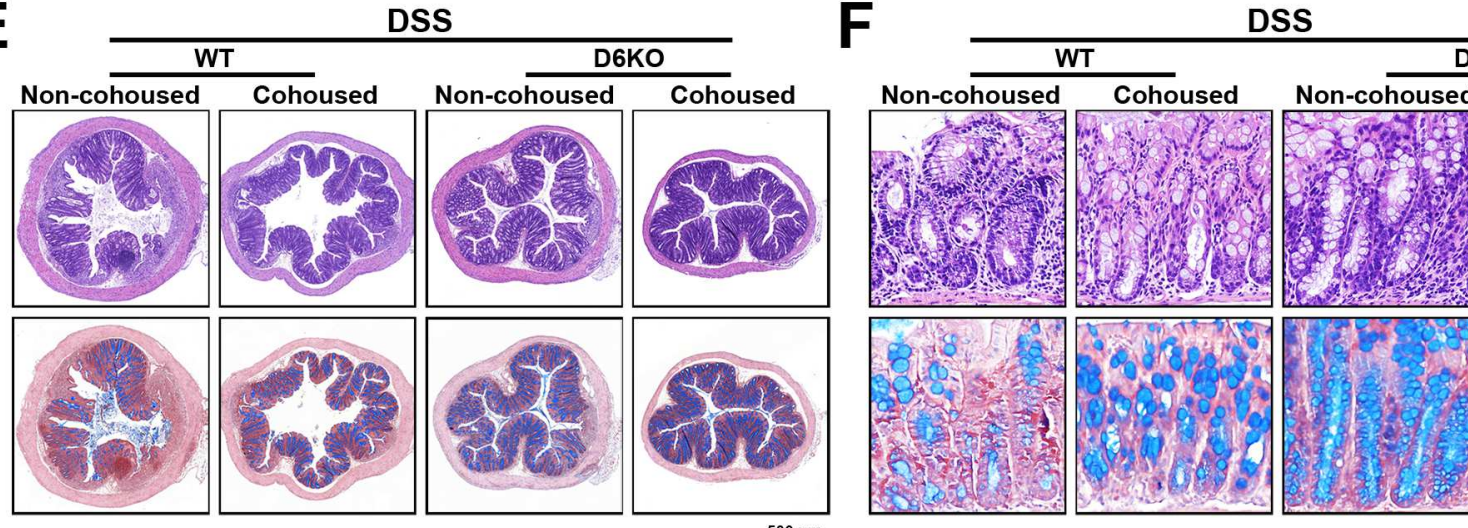

D6KO

G

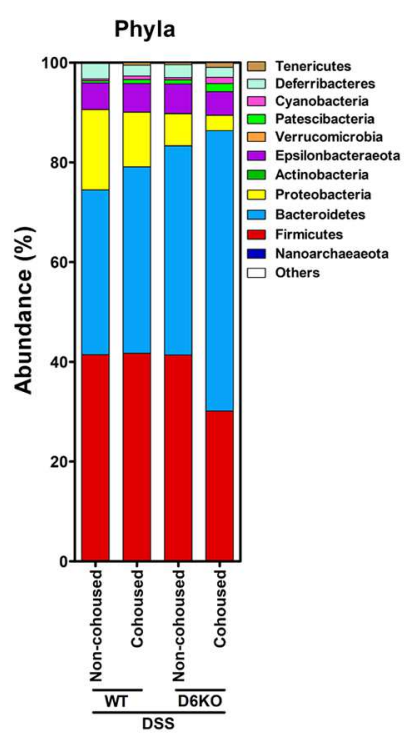

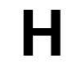

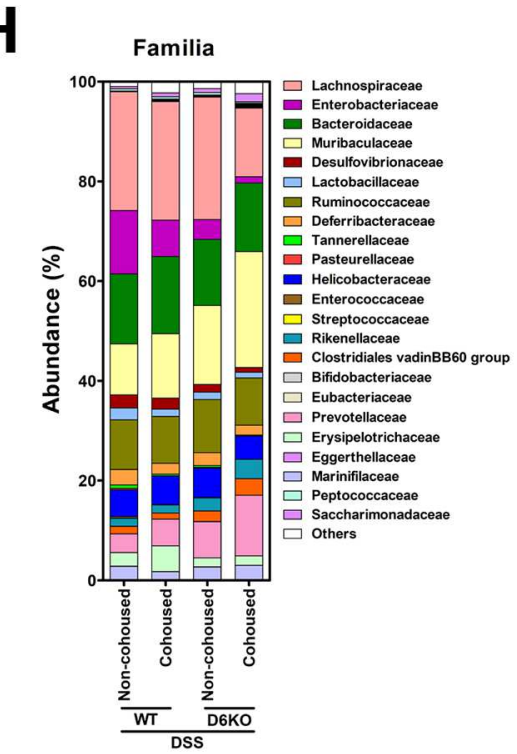

$\underline{0 \mathrm{~m}}$

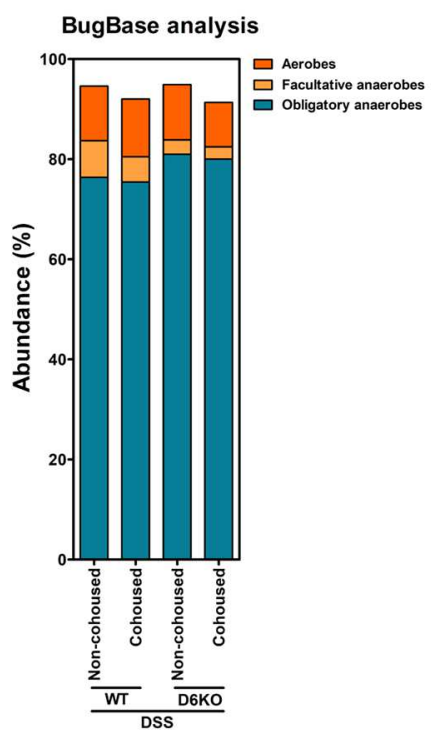


Figure 7

A

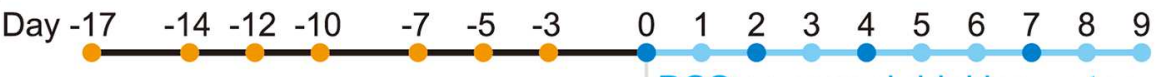

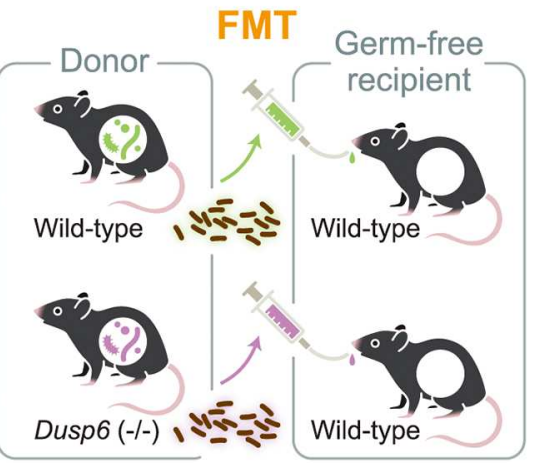

DSS or normal drinking water

B

C
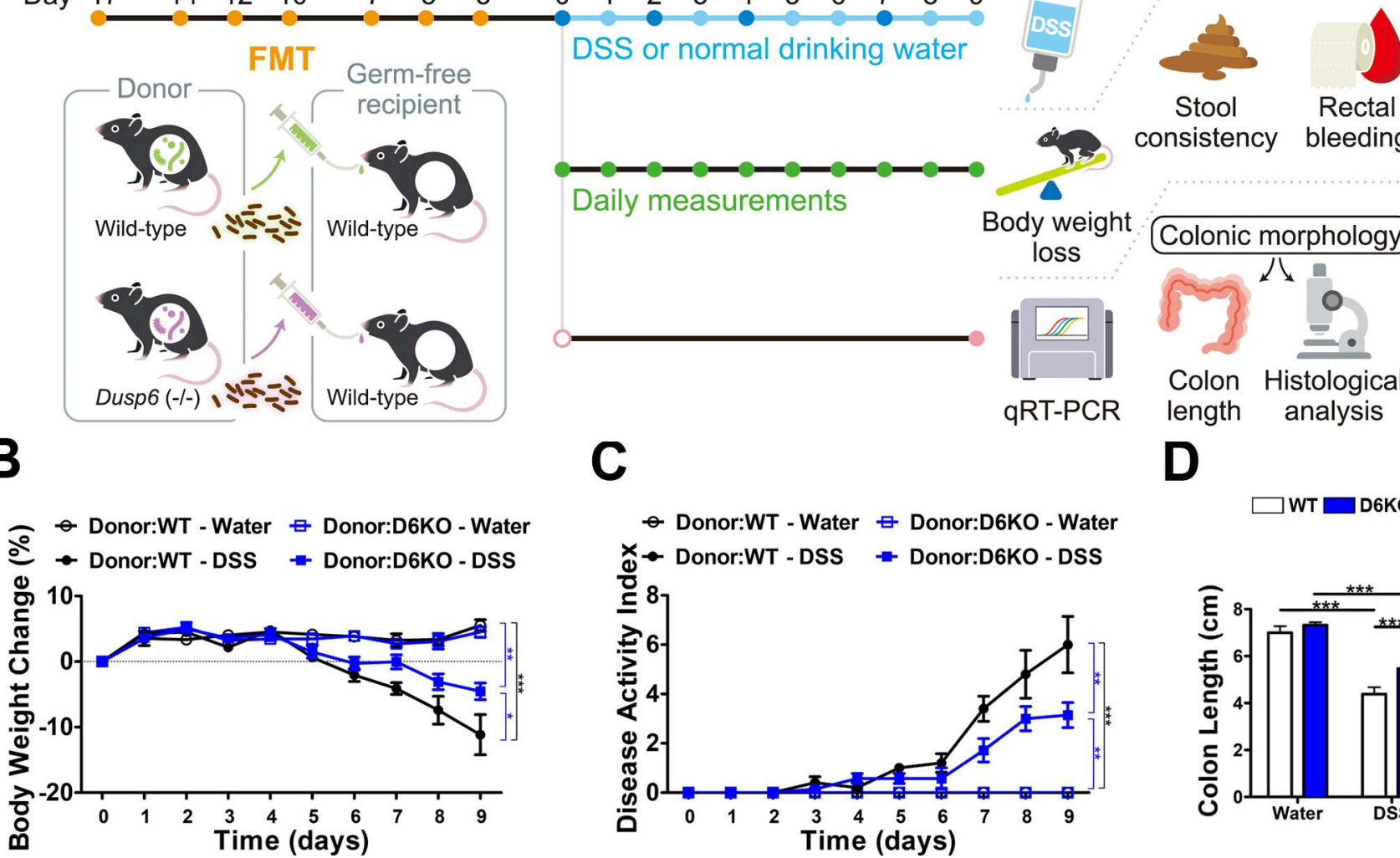

Stool Rectal consistency bleeding

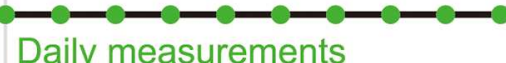

Body weight Colonic morphology
loss
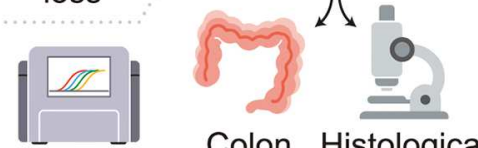

Colon Histological qRT-PCR length analysis

D

$\square$ WT $\square$ D6KO

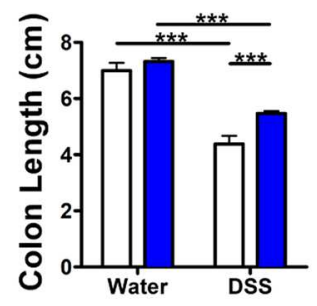

E

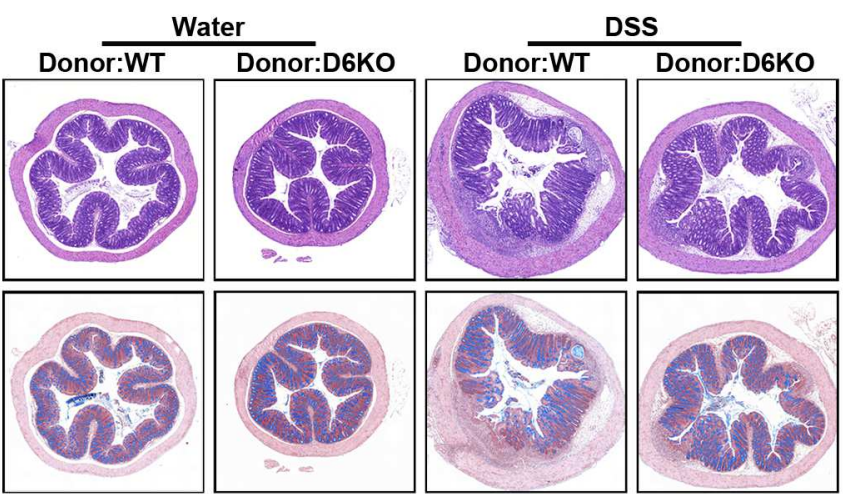

$\mathbf{F}$

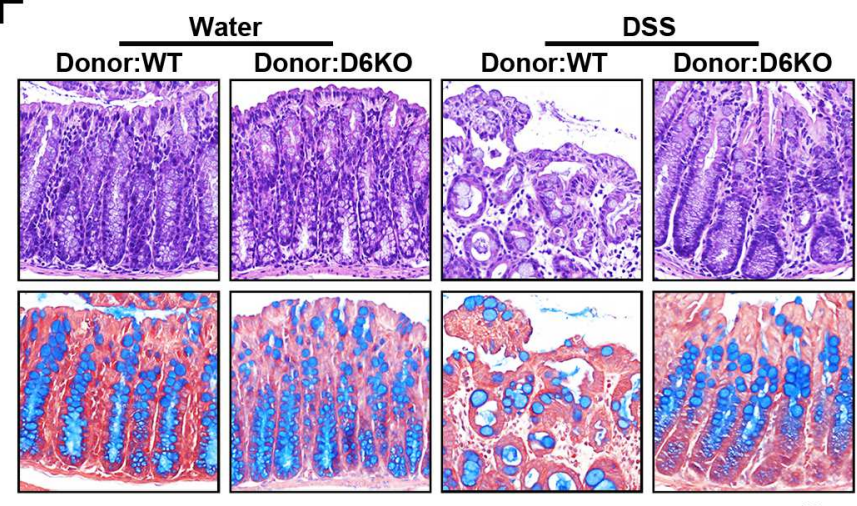

$\mathbf{G}$

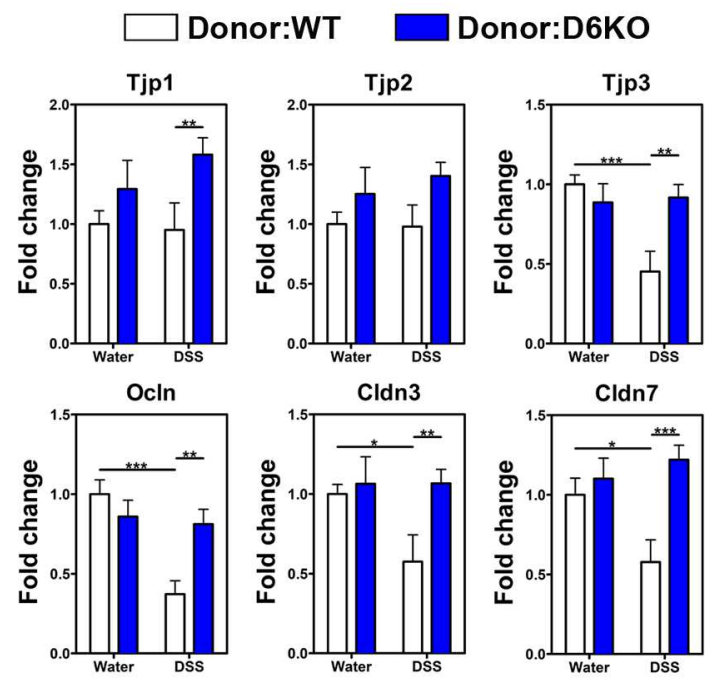

H
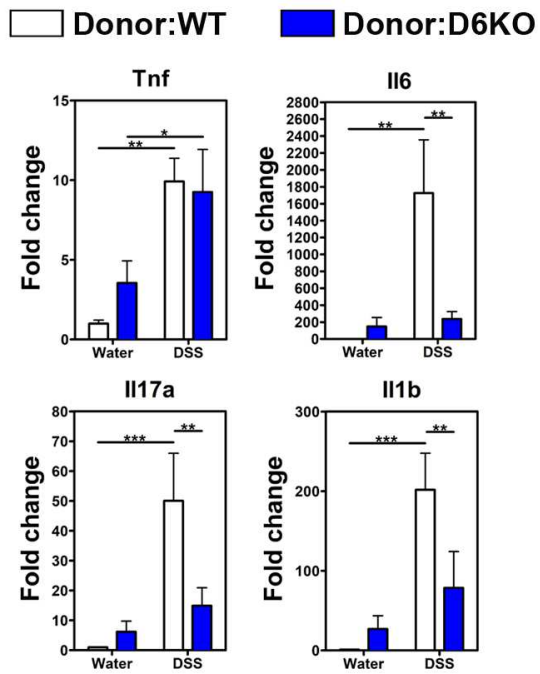
Figure 8

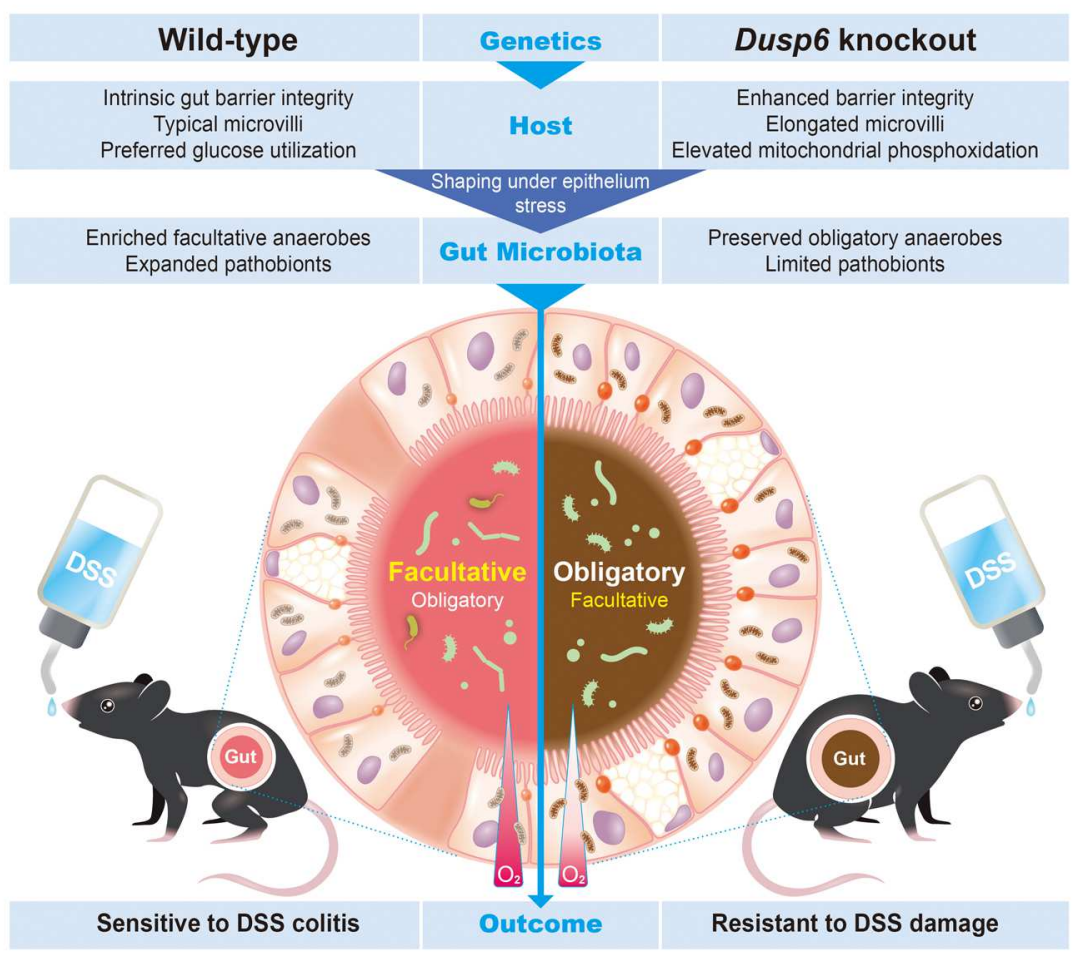




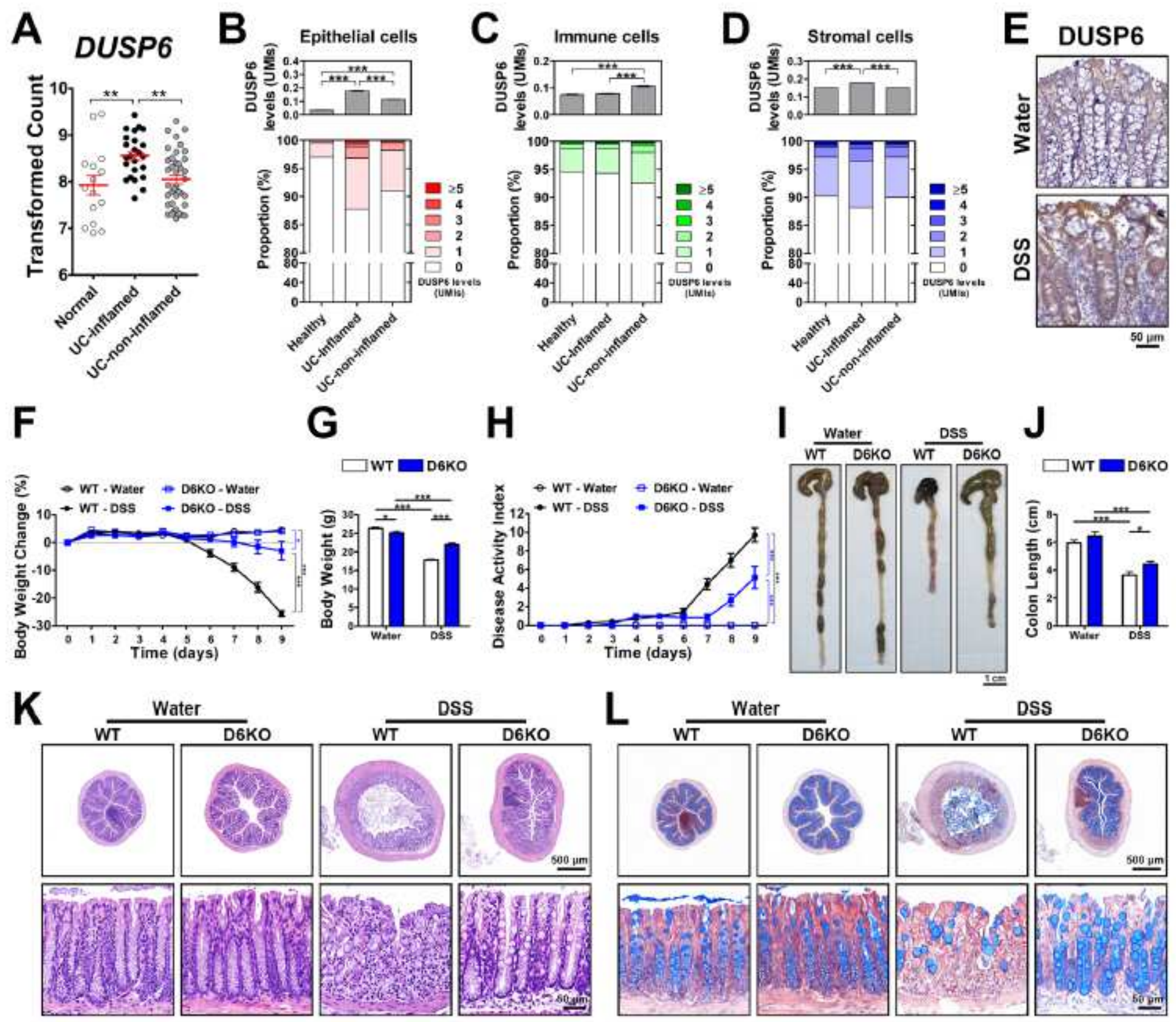

Figure 1

Dusp6 gene deficiency mitigated DSS-induced colitis. (A) DUSP6 expression levels in normal controls and UC patients (NCBI dataset GSE9452; normal: $n=5$; UC-inflamed: $n=8$; UC-noninflamed: $n=13$; each dot represents one microarray probeset for each group, with a total of 3 probesets mapped to DUSP6 in one microarray chip). ${ }^{*} \mathrm{P}<0.01$, Fisher's LSD test. $(B, C, D)$ Composition of cells with diverse levels of DUSP6 mRNA expression, as defined by the number of unique molecular identifiers (UMIs), in epithelial, immune, or stromal cells from colonic biopsies of healthy subjects and inflamed and non-inflamed tissues of UC patients (Single Cell Portal: SCP259). ${ }^{\star \star \star} P<0.001$, Mann-Whitney U test. (E) Immunohistochemical staining of DUSP6 protein (brown) in colonic tissue from C57BL/6J mice after 9 days of treatment with 
water or water plus DSS (indicated as DSS throughout). (F) Daily records of changes of body weight of wild-type (WT) and Dusp6 gene knockout (D6KO) mice during 9 days of treatment with water or DSS. ${ }^{*} P<0.05$ and ${ }^{* * *} P<0.001$, Fisher's LSD test. (G) The weights of WT and D6KO mice with or without DSS treatment. ${ }^{*} P<0.05$ and ${ }^{* * *} P<0.001$, Fisher's LSD test. $(H)$ Daily disease activity index scores of WT and D6KO mice transverse sections of colon tissue (top row) and colonic epithelium (bottom row; scale bar = $50 \mu \mathrm{m}$ ) from WT and D6KO mice after 9 days of treatment with water or DSS. (L) Representative Alcian blue stained transverse sections of colon tissue (top row; scale bar $=500 \mu \mathrm{m}$ ) and colonic epithelium (bottom row; scale bar $=50 \mu \mathrm{m}$ ) from WT and D6KO mice after 9 days of treatment with water or DSS. D6KO, Dusp6 gene knockout mice; DSS, dextran sulfate sodium; H\&E, hematoxylin and eosin; UC, ulcerative colitis; WT, wild-type mice. during 9 days of treatment with water or DSS. ${ }^{\star \star *} P<0.001$, Fisher's LSD test. Number of mice per group in F-H: WT-water, $n=6$; D6KO-water, $n=6$; WT-DSS, $n=7$; D6KO-DSS, $\mathrm{n}=7$. $(\mathrm{I}, \mathrm{J})$ Representative colon tissue and colon length in WT and D6KO mice after water or DSS treatment for 9 days. ${ }^{*} P<0.05$ and ${ }^{* * *} P<0.001$, Fisher's LSD test. Number of mice per group: WT-water, $n=$ 10; D6KO-water, $n=12$; WT-DSS, $n=11$; D6KO-DSS, $n=15$. (K) Representative H\&E stained 

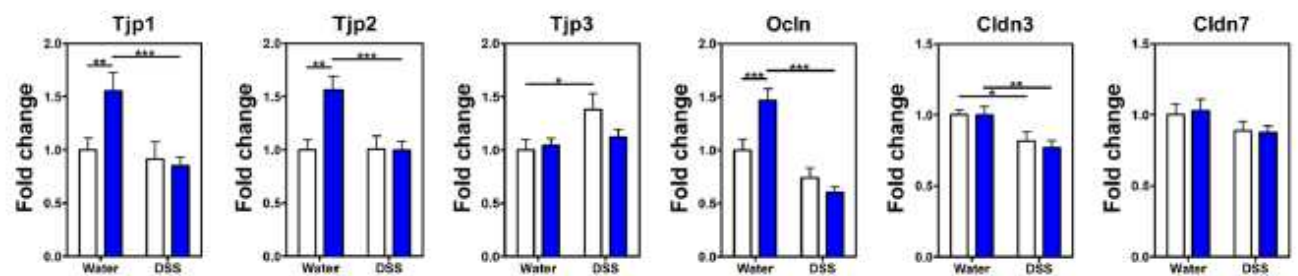

B

TJP2/DAPI

TJP3/DAPI
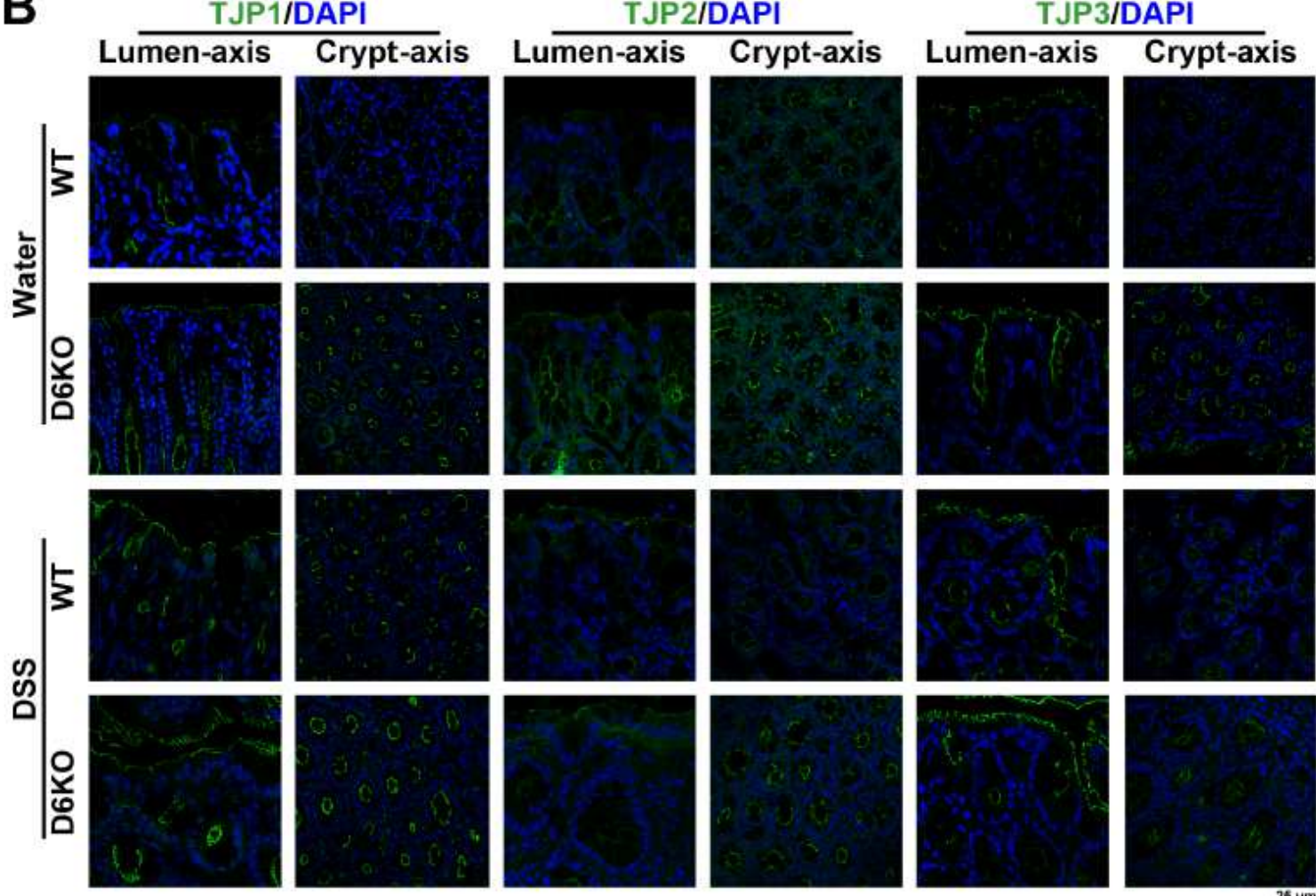

E
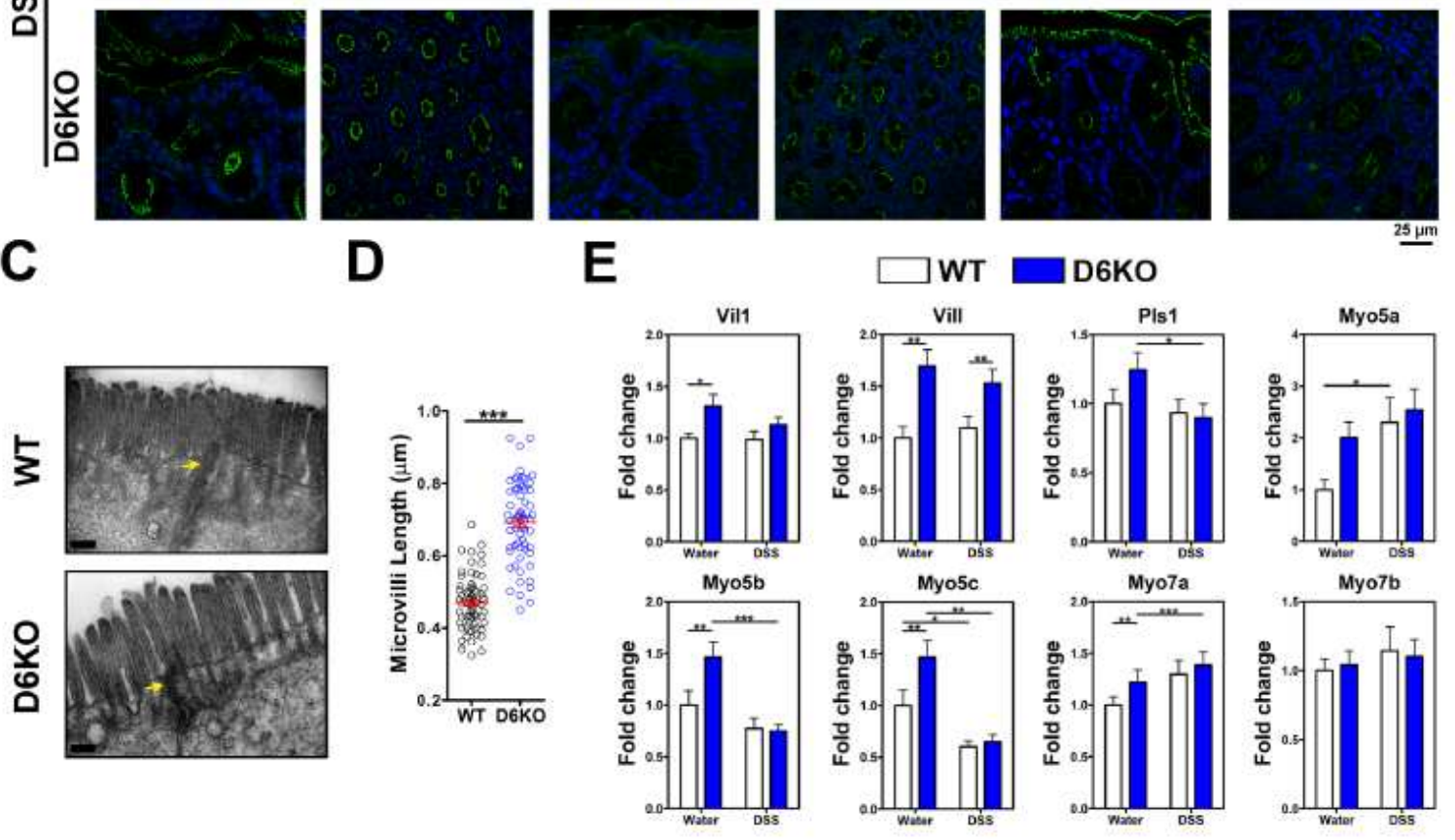

Figure 2

Dusp6 deficiency led to increased expression of tight junction proteins and elongated microvilli in mouse colon tissue. (A) qRT-PCR analysis of tight junction-associated genes in the colonic tissues of WT and D6KO mice after 9 days of treatment with water or water plus DSS (indicated as DSS throughout). ${ }^{*} \mathrm{P}<0.05,{ }^{*} \mathrm{P}<0.01$, and ${ }^{* * *} \mathrm{P}<0.001$, Fisher's LSD test. Number of mice per group: WT-water, $\mathrm{n}=10$; D6KOwater, $n=12$; WT-DSS, $n=11$; D6KO-DSS, $n=15$. (B) Immunofluorescence staining of TJP1, TJP2, and 
TJP3 (green) in colon tissue from WT and D6KO mice treated with water or DSS for 9 days. Images of the colonic epithelium along luminal and crypt axes were captured by confocal microscopy. Cell nuclei were stained with DAPI (blue). (C) Ultrastructural changes in the tight junctions and microvilli of the colonic epithelium in WT and D6KO mice via transmission electron microscopy. The tight junction complexes are indicated by yellow arrows. Scale bar $=200 \mathrm{~nm}$. (D) Quantitative analysis of colonic epithelial cell microvilli length in WT and D6KO mice. ${ }^{* \star *} \mathrm{P}<0.001$, Student's t-test. Number of microvilli per group: WT, $\mathrm{n}$ = 66; D6KO, $n=62$. (E) qRT-PCR analysis of microvilli-associated genes in the colonic tissue of WT and D6KO mice after 9 days of treatment with water or DSS. ${ }^{*} P<0.05$, ${ }^{*} P<0.01$, and ${ }^{*} * * P<0.001$, Fisher's LSD test. Number of mice per group: WT-water, $n=10$; D6KO-water, $n=12$; WT-DSS, $n=11$; D6KODSS, $n=15$. D6KO, Dusp6 knockout mice; DSS, dextran sulfate sodium; WT, wild-type mice. 
A

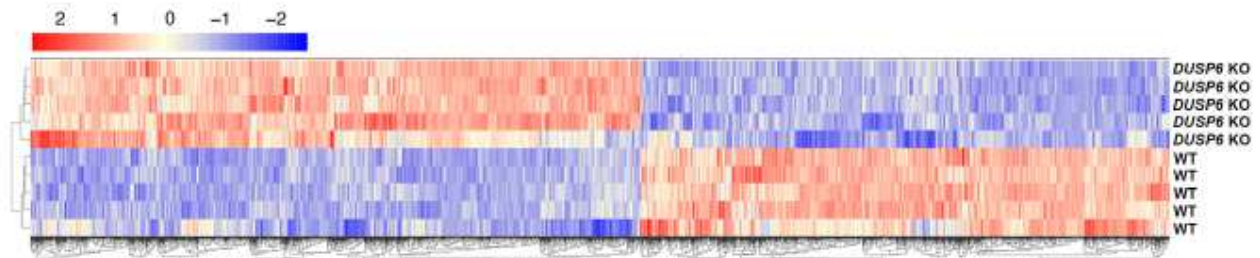

B

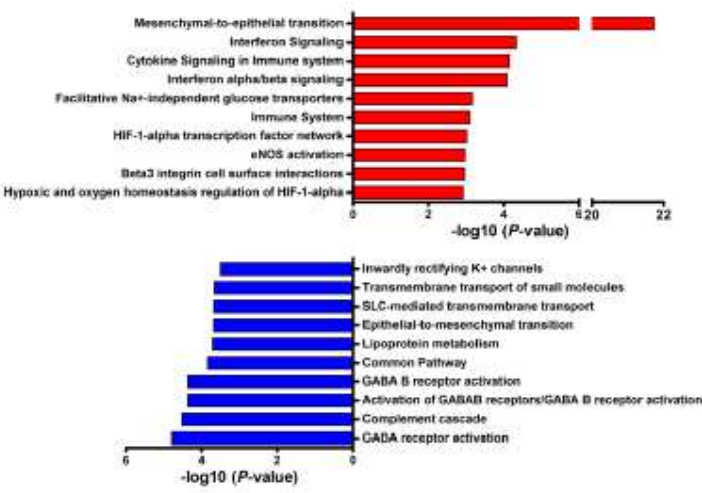

C

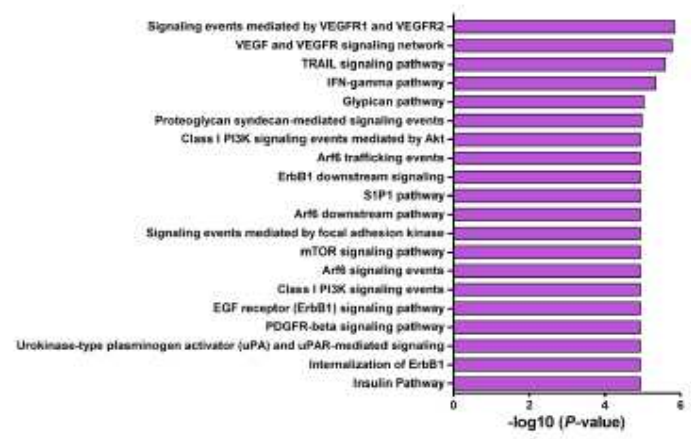

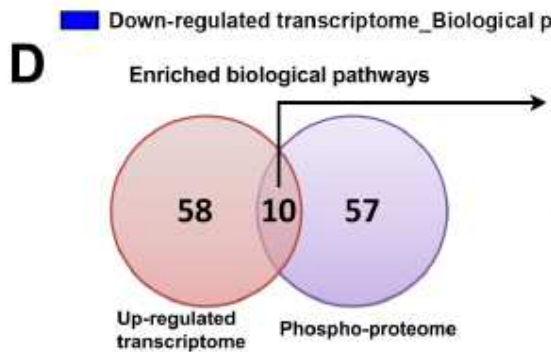

$E^{\text {TJP1 }}$
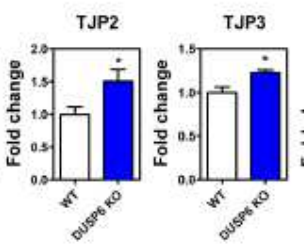

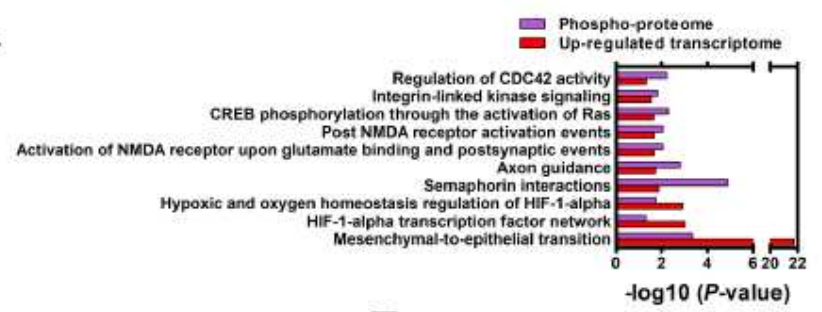

G

TJP1
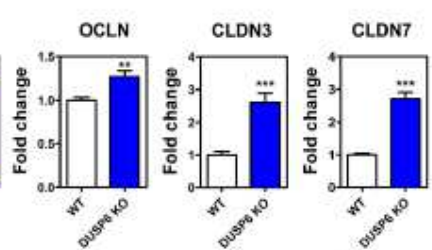

F

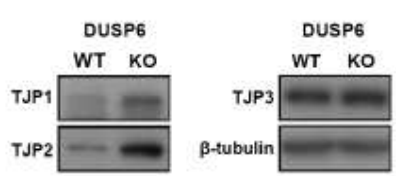

H

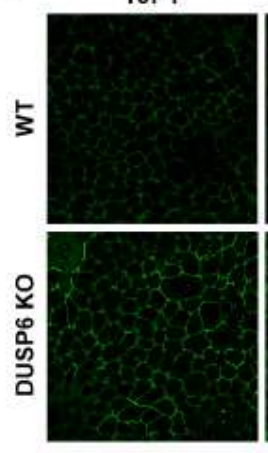

TJP2

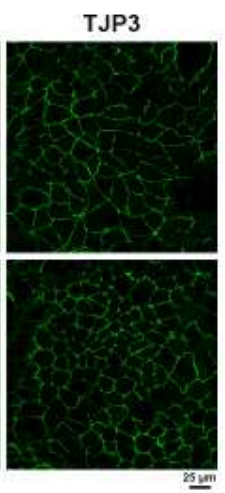

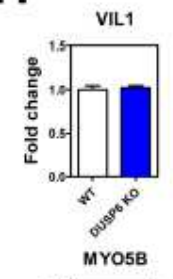
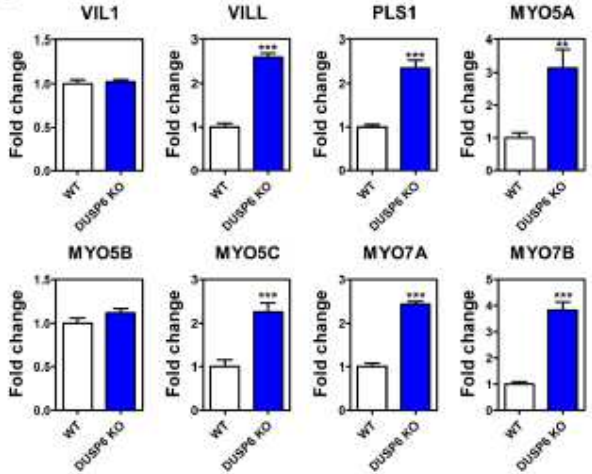

\section{Figure 3}

DUSP6 knockout altered the transcriptome and phospho-proteome in human gut epithelial Caco-2 cells. (A) The heatmap of DEGs from RNA-seq analysis performed in WT and DUSP6 gene knockout (DUSP6 KO) Caco-2 cells after 21 days in culture ( $n=5$ in each group). The color coding indicates to relative gene expression level based on Z-score transformation. (B) Top 10 FunRich biological pathways of the transcriptome that were significantly up-regulated or down-regulated in DUSP6 KO compared to WT cells. 
(C) Top 20 FunRich biological pathways of the phosphoproteome that were significantly altered in DUSP6 KO compared to WT cells. (D) Intersection of upregulated transcriptomic and phospho-proteomic datasets shows the enriched biological pathways that could be modulated by DUSP6 deficiency. (E) qRTPCR analysis of tight junction protein genes in WT and DUSP6 KO cells after 21 days in culture. ${ }^{*}<<0.05$, ${ }^{\star *} P<0.01$, and ${ }^{* \star *} P<0.001$, Student's t-test; $n=5$ in each group. ( $\left.F\right)$ Western blotting of DUSP6, TJP1, TJP2, and TJP3 protein from WT and DUSP6 KO cells after 21 days in culture. (G) Immunofluorescent staining of TJP1, TJP2, and TJP3 proteins in WT and DUSP6 KO cells after 7 days in culture. (H) qRT-PCR analysis of microvilli-associated genes in WT and DUSP6 KO cells after 21 days in culture. ${ }^{*} \mathrm{P}<0.01$ and $\star \star * \mathrm{P}<0.001$, Student's t-test; $\mathrm{n}=5$ in each group.
A
B
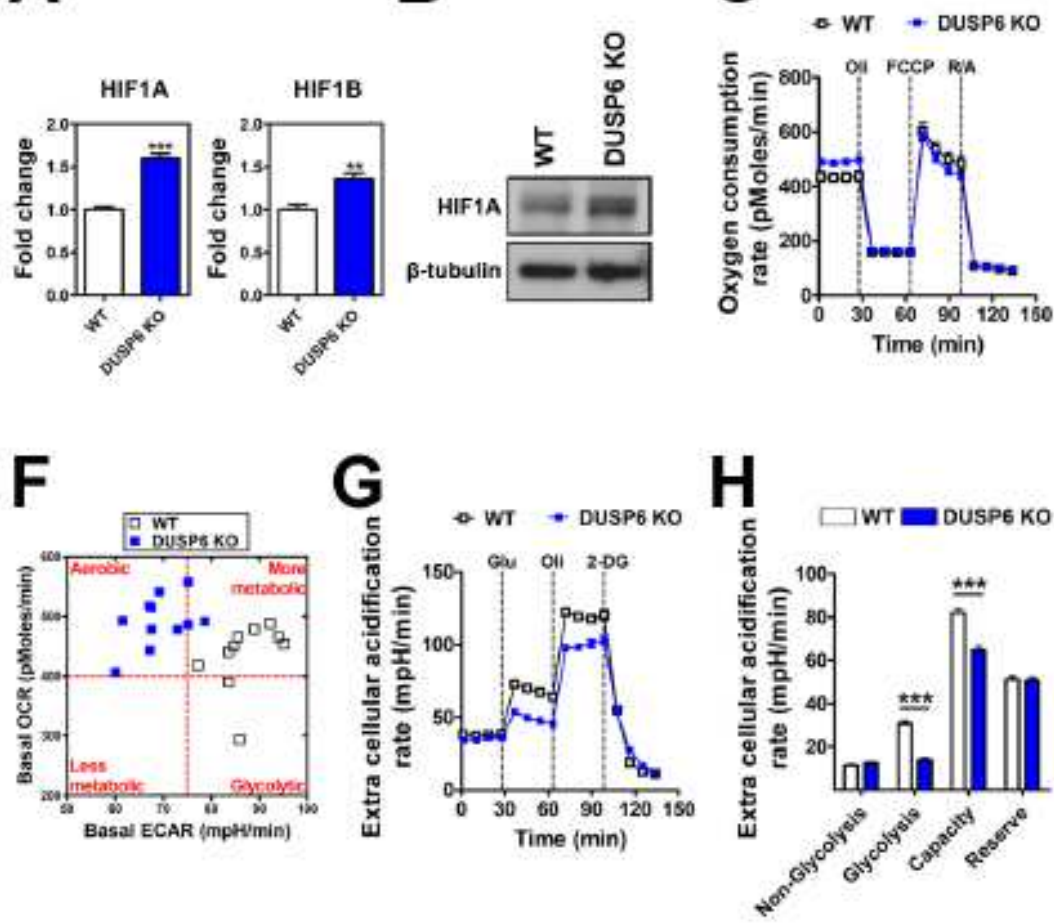
D

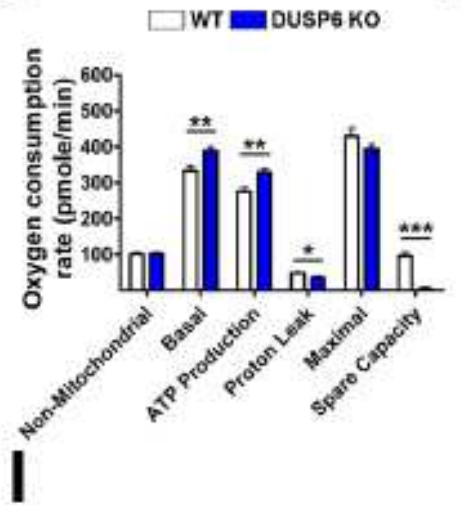
E
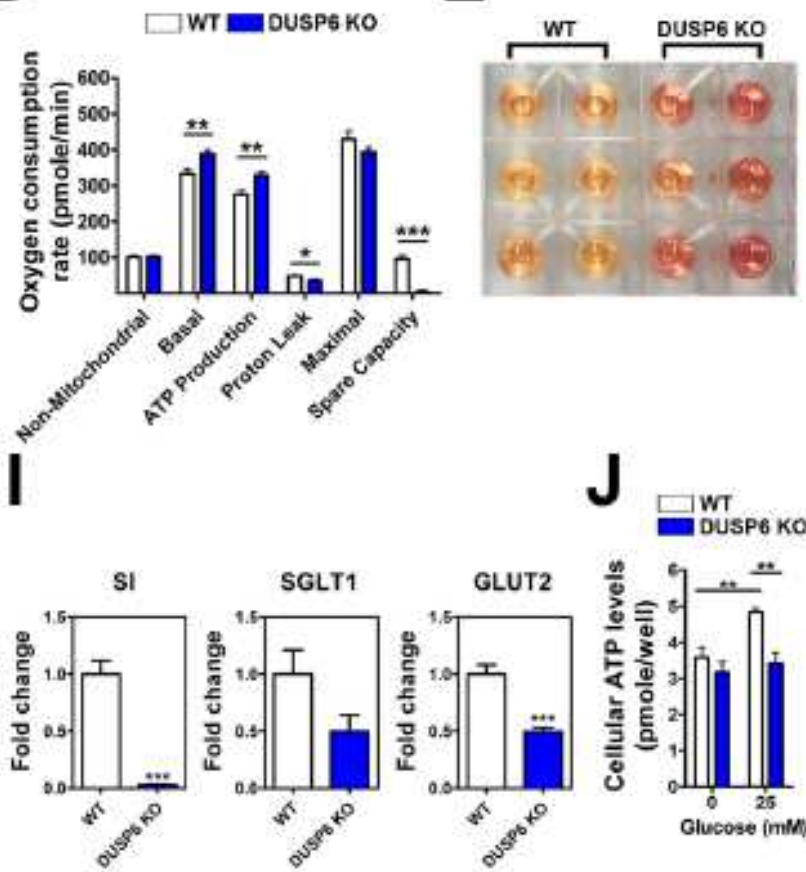

\section{Figure 4}

DUSP6 knockout elevated mitochondrial oxidation and attenuated glycolysis in Caco-2 cells. (A) mRNA levels of hypoxia-associated genes in WT and DUSP6 KO cells after 21 days in culture. ${ }^{* * P}<0.01$ and $\star \star \star P<0.001$, Student's t-test; $n=5$ in each group. (B) Western blotting of HIF1A protein from WT and DUSP6 KO cells after 21 days in culture. $\beta$-tubulin protein was used as a loading control. (C) Real-time oxygen consumption rate (OCR) values from WT $(n=10)$ and DUSP6 KO $(n=11)$ cells in response to the addition of oligomycin (Oli), carbonyl cyanide-ptrifluoromethoxyphenylhydrazone (FCCP) and rotenone plus antimycin A (R/A). (D) Statistical analysis of non-mitochondrial respiration, basal respiration, ATP production, proton leak, maximal respiration, and spare capacity based on oxygen consumption rate values. ${ }^{*} P<0.05,{ }^{*} \times<<0.01$, and ${ }^{*} * * P<0.001$, Student's t-test. (E) Color change of phenol red-containing medium after incubation with WT and DUSP6 KO cells for 3 days. Image was taken on day 21 of cell culture. (F) Plot of basal OCR versus extracellular acidification rates (ECARs) of WT ( $n=10)$ and DUSP6 KO $(n=11)$ cells in glucose-containing medium. $(G)$ Real-time ECARs of WT $(n=10)$ and DUSP6 KO $(n=$ 
11) cells in response to the addition of glucose (Glu), oligomycin (Oli), and 2-deoxy-D-glucose (2-DG). (H) Statistical analysis of non-glycolytic acidification, glycolysis, glycolytic capacity, and glycolytic reserve based on ECAR values. ${ }^{* *} \mathrm{P}<0.001$, Student's t-test. (I) qRT-PCR analysis of glucose uptake genes in WT and DUSP6 KO cells after 21 days in culture. ${ }^{* *} \mathrm{P}<0.001$, Student's t-test; $\mathrm{n}=5$ in each group. $(\mathrm{J})$ Glucose utilization by WT and DUSP6 KO Caco-2 cells via evaluation of cellular ATP levels after supplementation with glucose for 4 hrs. ${ }^{*} P<0.01$, Student's t-test; $n=5$ in each group. DUSP6 KO, DUSP6 gene knockout Caco-2 cells; ECAR, extracellular acidification rate; OCR, oxygen consumption rate; WT, wild-type Caco-2 cells.
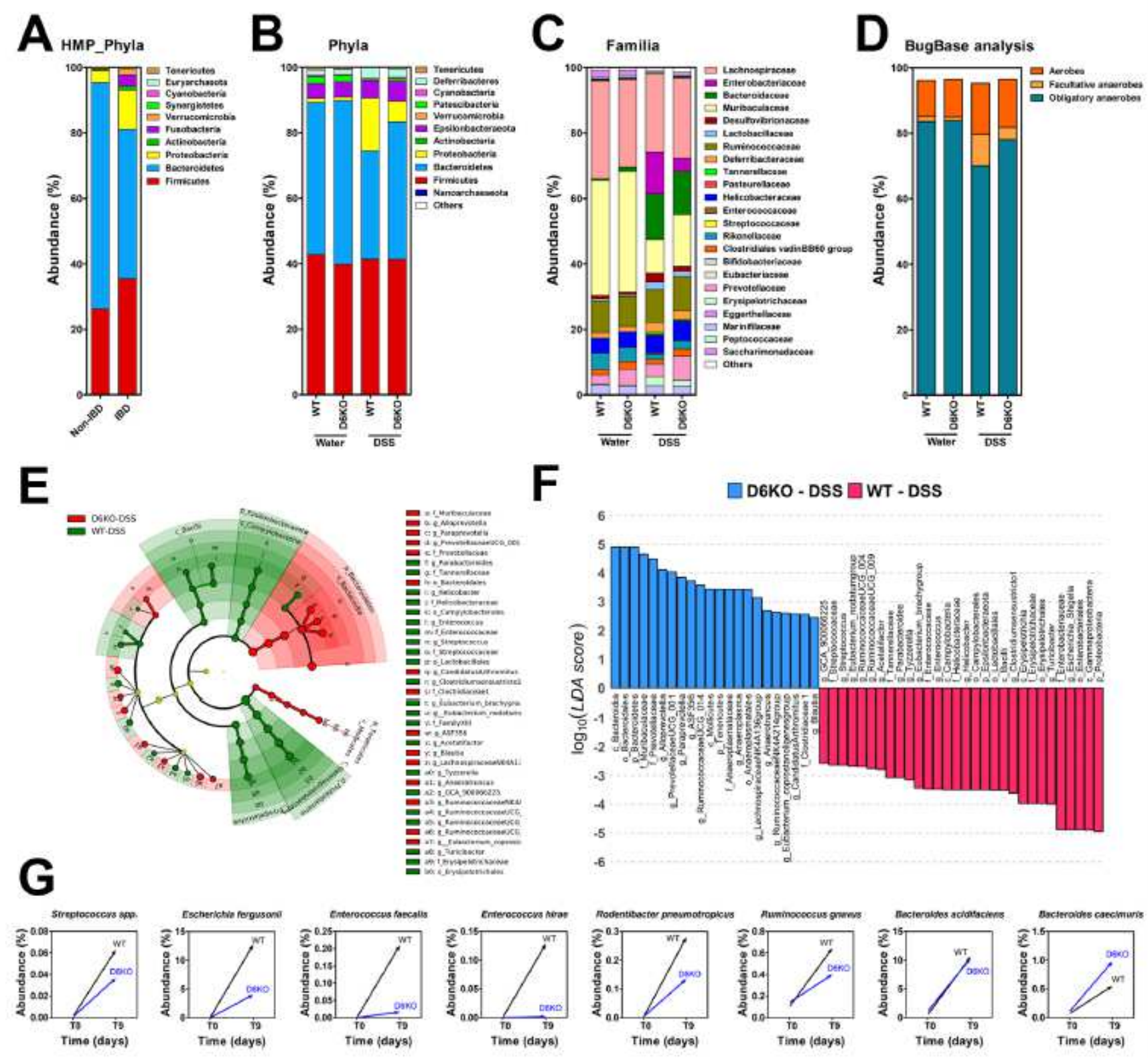

Figure 5

Comparison of fecal microbiota derived from WT and D6KO mice before and after DSS treatment. (A) Phylum-level relative abundance of fecal microbiota of IBD and non-IBD subjects reanalyzed from the HMP dataset (IBD, $n=57$; non-IBD, $n=320$ ). (B, C) Composition of fecal microbiota, at phylum and family 
levels, from WT and D6KO mice before or after 9 days of DSS treatment. (D) BugBase analysis of the proportion of aerobes, facultative anaerobes, and obligate anaerobes in mouse gut. (E) Cladograms generated from LEfSe analysis showing the microbial clades with the greatest differences in abundance in microbiota from WT (green) or D6KO (red) mice after DSS treatment. (F) LDA scores of microbial clades differing in abundance between WT and D6KO mice after DSS treatment (with LDA score $>2$ and significance of $a<0.05$, determined by Kruskal-Wallis test). (G) Comparison of changes in bacterial species in WT and D6KO mice after DSS treatment. Values are means for each group. Number of mice per group: WT-water, $n=48$ and D6KO-water, $n=51$; WT-DSS, $n=22$ and D6KO-DSS, $n=28$. D6KO, Dusp6 gene knockout mice; DSS, dextran sulfate sodium; HMP, human microbiome project; IBD, inflammatory bowel disease; LDA, linear discriminant analysis; WT, wild-type mice. 


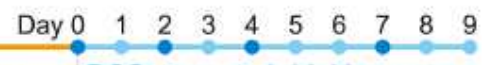
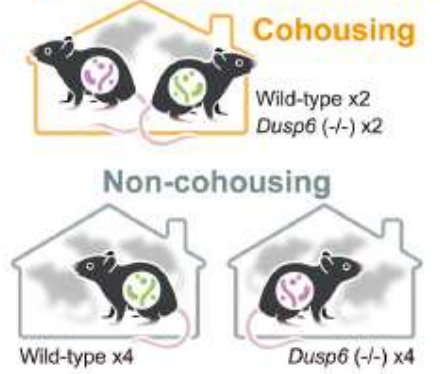

B

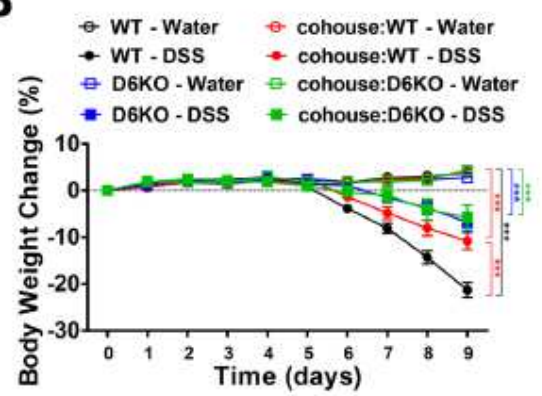

DSS or normal drinking water

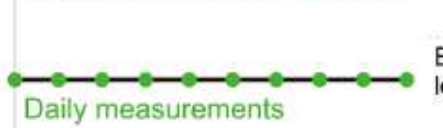

16S rRNA gut microbiome

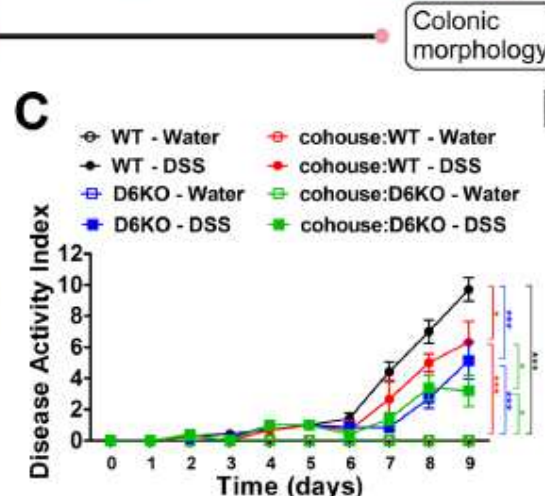

Stool consistency

Body weight Rectal

bleeding

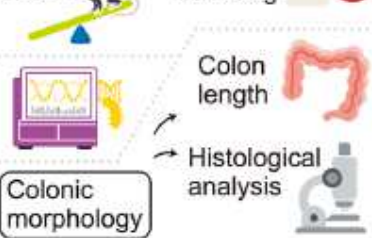

D $\square$ wт.Dss

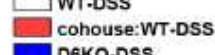

DGKo-Dss

cohouse:D6KO-DSS

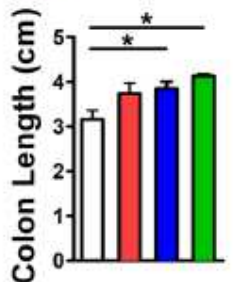

\section{E}

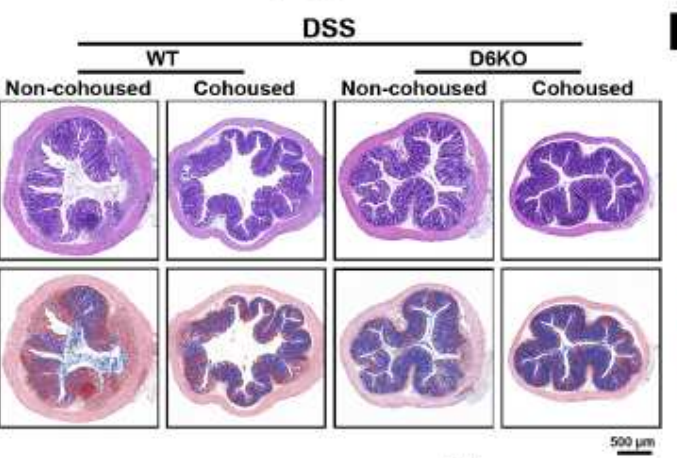

G

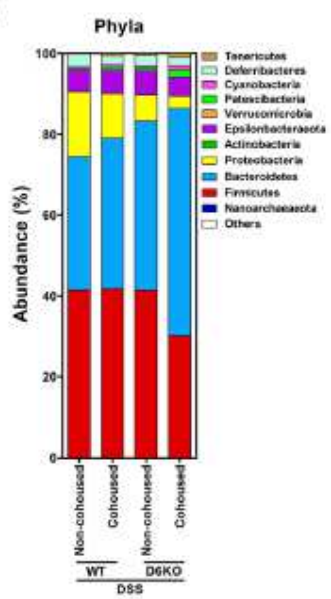

H

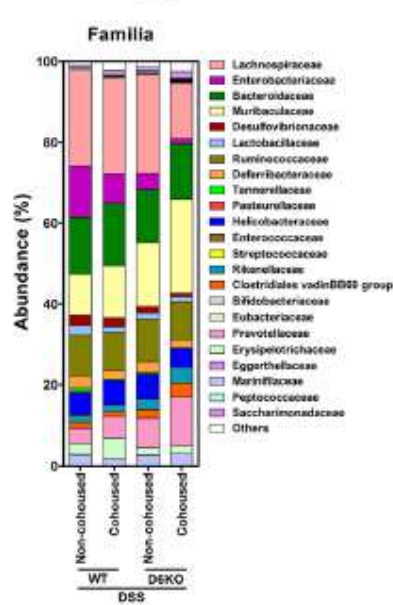

DSS

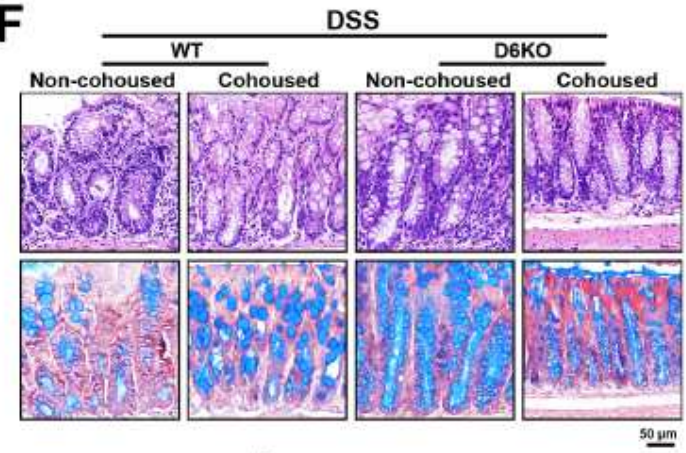

I

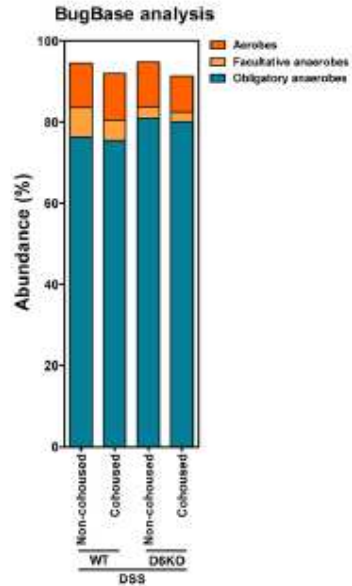

Figure 6

Cohousing of WT mice with D6KO mice mitigated DSS-induced colitis. (A) Schematic of the cohousing experiment. Two D6KO mice and two WT littermates were housed together for 4 weeks, followed by 9 days of treatment with DSS. (B, C) Daily weight change and disease activity index scores for WT and D6KO mice, with or without cohousing, during 9 days of treatment with water or water plus DSS (indicated as DSS throughout). ${ }^{\star} P<0.05,{ }^{\star \star} \mathrm{P}<0.01$, and ${ }^{\star \star *}{ }^{*} \mathrm{P}<0.001$, Fisher's LSD test. (D) Colon length in 
WT and D6KO mice, with or without cohousing, after 9 days of treatment with DSS. ${ }^{*}<0.05$ and $\star \star * P<0.001$, Fisher's LSD test. Number of mice per group in B-D: Noncohoused WT-water, $n=13$; Noncohoused D6KO-water, $n=14$; Non-cohoused WT-DSS, $n=15$; Non-cohoused D6KO-DSS, $n=15$; Cohoused WT-water, $n=7$; Cohoused D6KO-water, $n=8$; Cohoused WT-DSS, $n=7$; Cohoused D6KO-DSS, $\mathrm{n}=9$. (E) Representative H\&E (top row) or Alcian blue (bottom row) stained transverse sections of colon tissue from the WT and D6KO mice, with or without co-housing, after 9 days of DSS treatment. (F) Representative images of colonic epithelium stained with H\&E (top row) or Alcian blue (bottom row). (G, H) Composition of the fecal microbiota of DSS-treated WT and D6KO mice, with or without cohousing, at the phylum and family levels. (I) The proportion of aerobes, facultative anaerobes and obligate anaerobes in mouse gut analyzed via BugBase. Number of mice per group in G-I: Non-cohoused WT-DSS, $\mathrm{n}=22$; Cohoused WT-DSS, $\mathrm{n}=8$; Non-cohoused D6KO-DSS, $\mathrm{n}=28$; Cohoused D6KO-DSS, $\mathrm{n}=8$. D6KO, Dusp6 gene knockout mice; DSS, dextran sulfate sodium; H\&E, hematoxylin and eosin; WT, wild-type mice. 
A

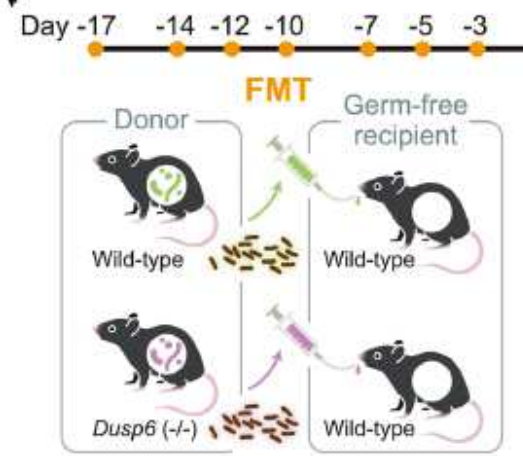

B

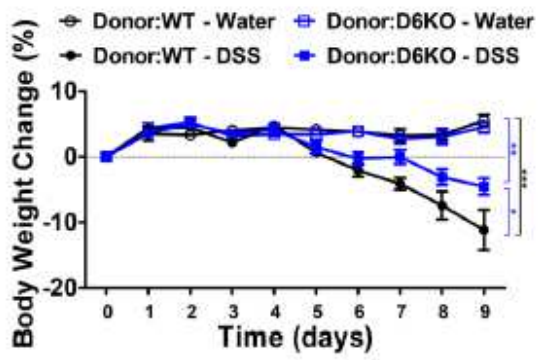

\section{C}
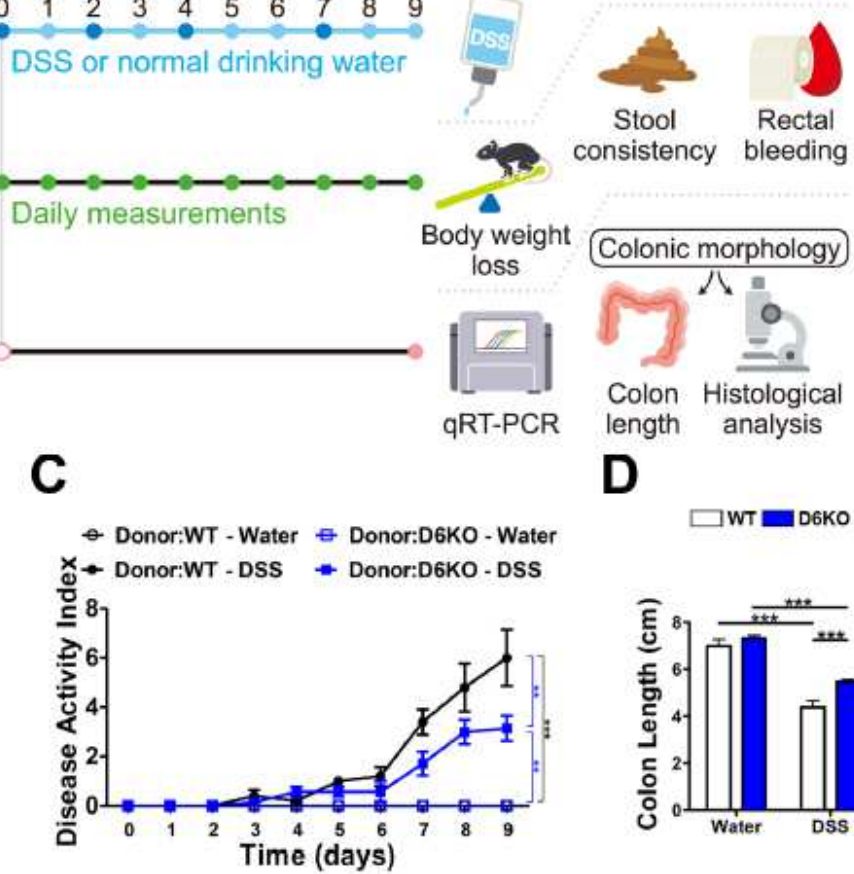

D
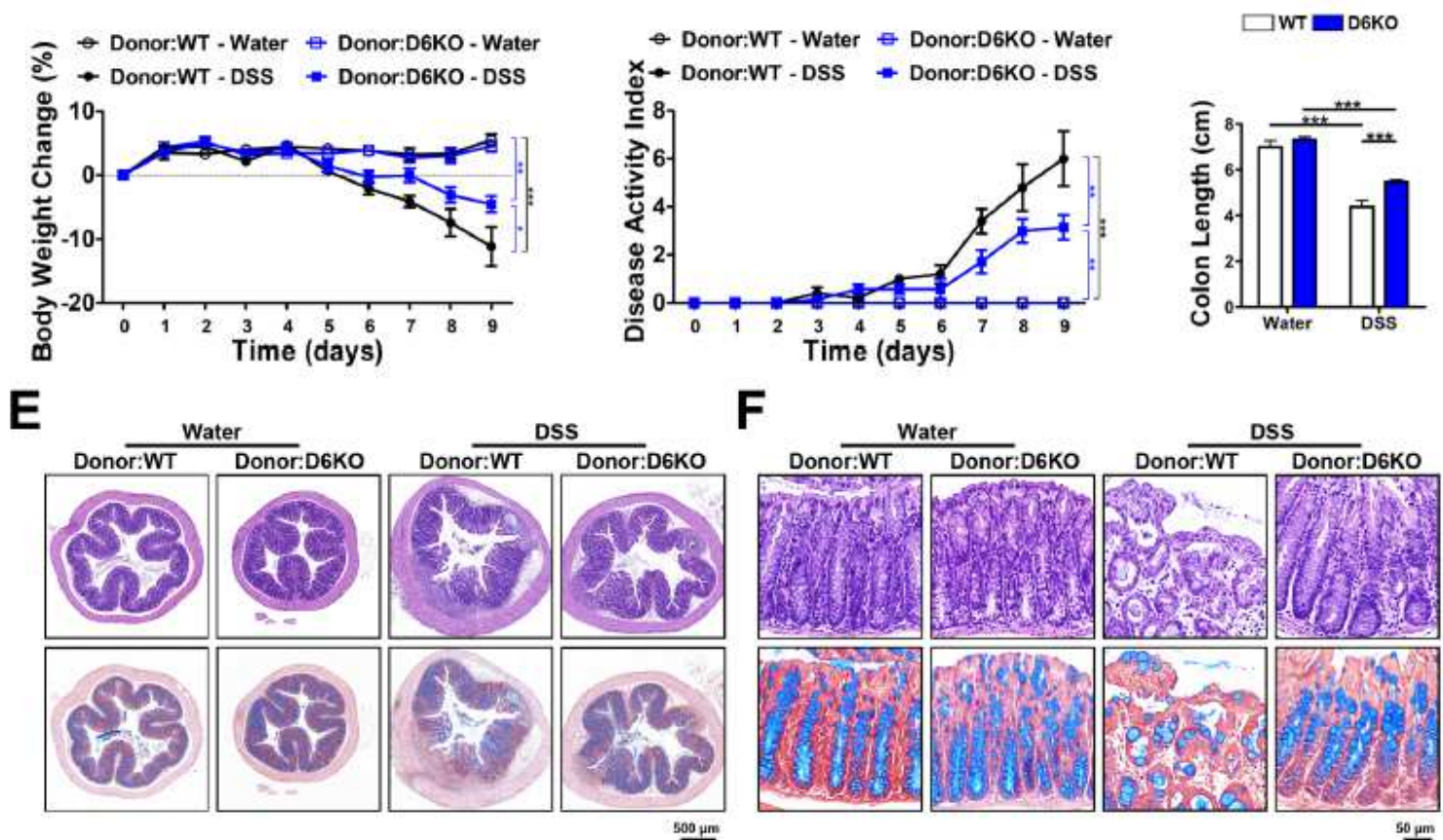

$\mathbf{F}$

G

$\square$ Donor:WT Donor:D6KO

H
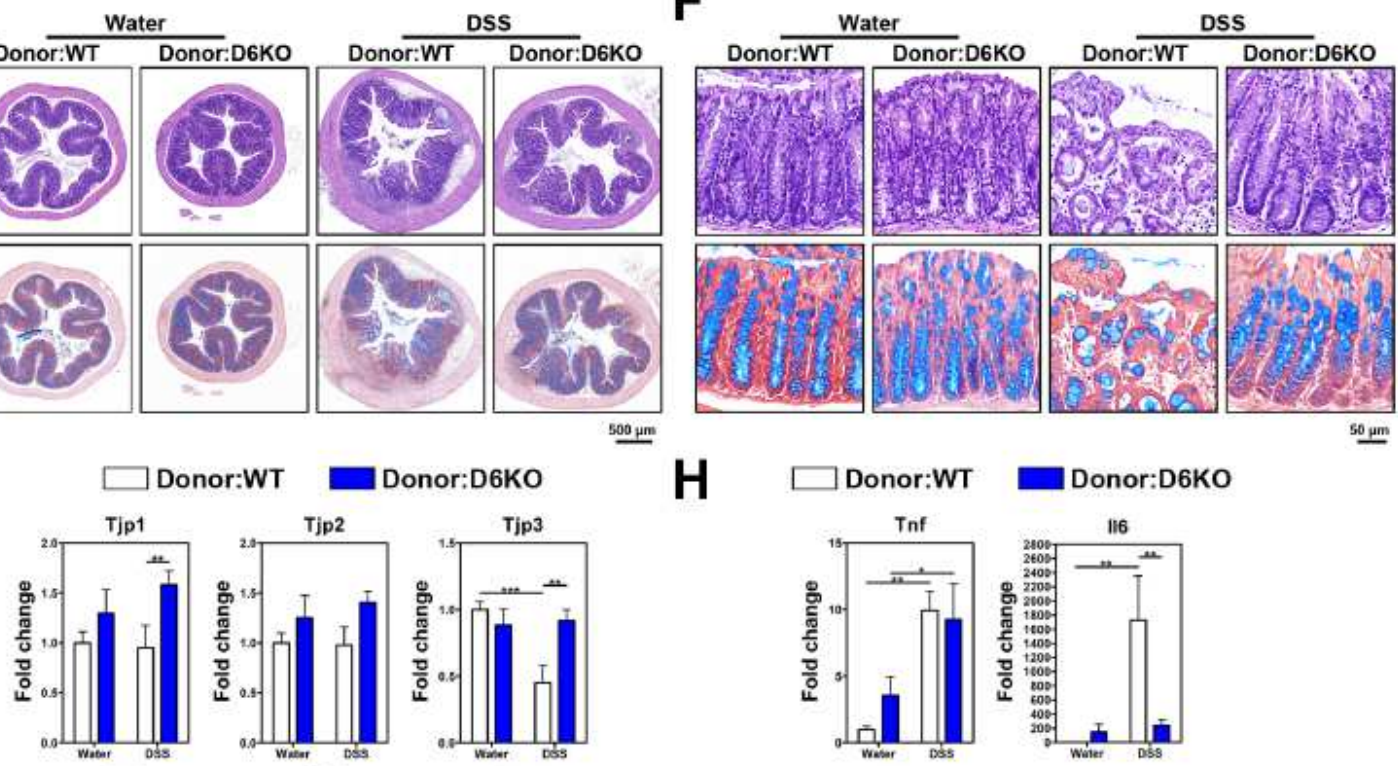

Donor:D6ko
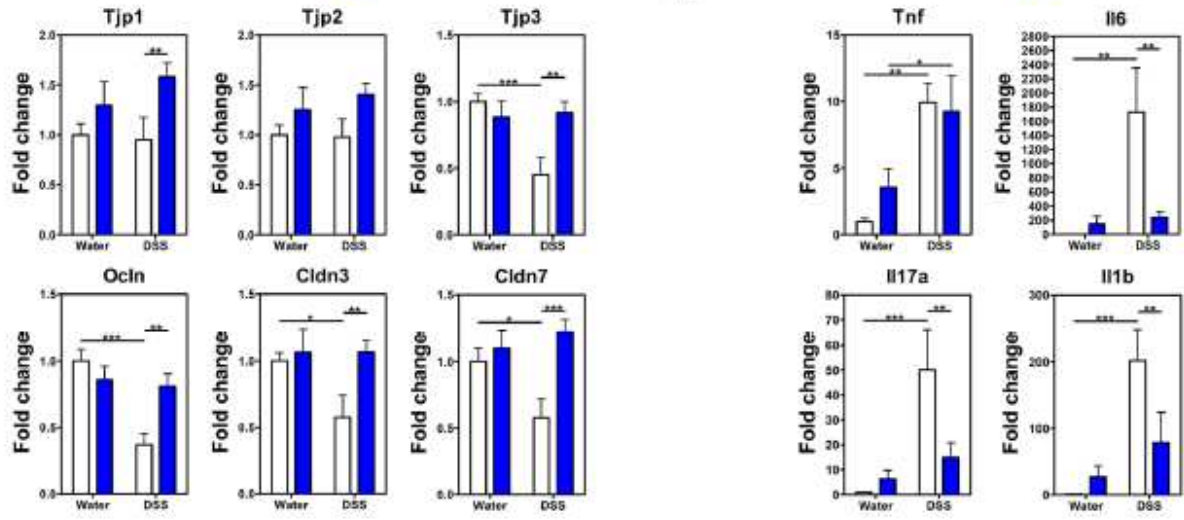

\section{Figure 7}

Fecal microbiota derived from D6KO mice mitigated DSS-induced colitis. (A) Schematic of the FMT experiment. The fecal microbiota were collected from non-cohoused WT or D6KO mice within 30 min and transferred by oral gavage into germ-free mice three times per week. After the seventh FMT, the mice were rested for 3 days and then treated with DSS for 9 days. (B) Daily change in body weight was recorded for germ-free mice transplanted with fecal microbiota from WT or D6KO mice during 9 days of treatment 
with water or water plus DSS (indicated as DSS throughout). ${ }^{*} P<0.05,{ }^{\star *} P<0.01$, and ${ }^{\star *}{ }^{*} P<0.001$, Fisher's LSD test. (C) Daily disease activity index scores of germ-free mice transplanted with gut microbiota from WT or D6KO mice during 9 days of treatment with water or DSS. ${ }^{*} P<0.01$ and ${ }^{*} * * P<0.001$, Fisher's LSD test. Number of mice per group in B-C: Donor:WT-water, $n=4$; Donor:D6KO-water, $n=4$; Donor:WT-DSS, $n$ $=5$; Donor:D6KO-DSS, $n=7$. (D) Colon length in germ-free mice with gut microbiota from WT or D6KO mice during 9 days of treatment with water or DSS. ${ }^{* \star} P<0.001$, Fisher's LSD test. Number of mice per group: Donor:WT water, $n=7$; Donor:D6KO-water, $n=7$; Donor:WT-DSS, $n=9$; Donor:D6KO-DSS, $n=10$. (E) Representative H\&E (top row) or Alcian blue (bottom row) stained transverse sections of colon tissue from germ-free mice with fecal microbiota from WT or D6KO mice after 9 days of treatment with DSS. (F) Representative images of colonic epithelium stained with H\&E (top row) or Alcian blue (bottom row). (G, H) qRT-PCR analysis of colonic tissues from Donor:WT and Donor:D6KO mice for expression of tight junction-associated genes and inflammatory cytokine genes after 9 days of treatment with water or DSS. ${ }^{\star} \mathrm{P}<0.05,{ }^{\star} \mathrm{P}<0.01$, and ${ }^{\star *} \mathrm{P}<0.001$, Fisher's LSD test. Number of mice per group in $\mathrm{G}-\mathrm{H}$ : Donor:WT-water, $\mathrm{n}$ = 7; Donor:D6KO-water, $n=7$; Donor:WT-DSS, $n=8$; Donor:D6KO-DSS, $n=9$. D6KO, Dusp6 gene knockout mice; Donor:WT, germ-free mice with fecal microbiota transplantation from wild-type mice; Donor:D6KO, germ-free mice with fecal microbiota transplantation from Dusp6 knockout mice; DSS, dextran sulfate sodium; FMT, fecal microbiota transplantation; H\&E, hematoxylin and eosin; WT, wild-type mice.

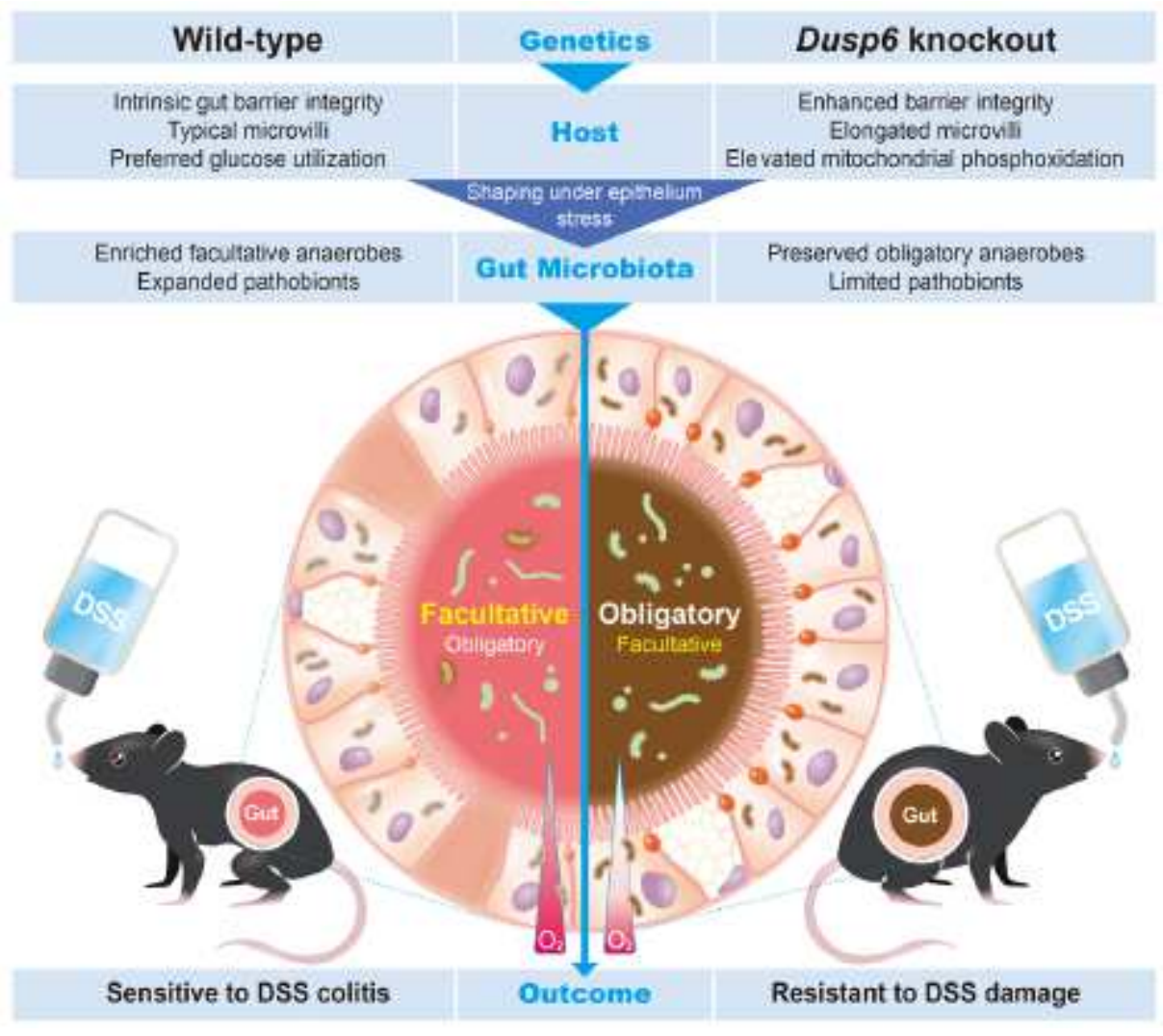

\section{Figure 8}

Dusp6 deficiency protects against DSS-induced colitis in mice. Dusp6 deficiency in mice results in induction of tight junction protein and microvilli-associated gene expression, which strengthens the connections between gut epithelial cells and extends the distance between microbes and the cytoplasm 
of enterocytes, thereby enhancing barrier integrity. Dusp6 deficiency also alters the cellular metabolic machinery, resulting in elevation of mitochondrial phosphorylation and reduction of glucose utilization. These changes maintain the gut lumen in an anaerobic state, which is an environment that promotes expansion of obligatory bacteria and limits the abundance of facultative bacteria and/or pathobionts. Therefore, Dusp6-deficient mice are resistant to DSSinduced epithelial damage and associated colitis.

\section{Supplementary Files}

This is a list of supplementary files associated with this preprint. Click to download.

- Supplementallnformation07162020BMCMicrobiomeCK.pdf

- SupplementalExcelTables.pdf 